\title{
Gamma-Ray Burst Progenitors
}

Levan, Andrew; Crowther, Paul; de Grijs, Richard; Langer, Norbert; Xu, Dong; Yoon, SungChul

Published in:

Space Science Reviews

DOI:

10.1007/s11214-016-0312-x

Publication date:

2016

Document version

Publisher's PDF, also known as Version of record

Citation for published version (APA):

Levan, A., Crowther, P., de Grijs, R., Langer, N., Xu, D., \& Yoon, S-C. (2016). Gamma-Ray Burst Progenitors.

Space Science Reviews, 202(1-4), 33-78. https://doi.org/10.1007/s11214-016-0312-x 


\title{
Gamma-Ray Burst Progenitors
}

\author{
Andrew Levan' ${ }^{1}$ Paul Crowther ${ }^{2}$ - Richard de Grijs ${ }^{3,4}$. \\ Norbert Langer ${ }^{5}$ Dong Xu ${ }^{6,7,8}$ • Sung-Chul Yoon ${ }^{9}$
}

Received: 26 August 2016 / Accepted: 8 November 2016 / Published online: 21 November 2016

(C) The Author(s) 2016. This article is published with open access at Springerlink.com

\begin{abstract}
We review our current understanding of the progenitors of both long and short duration gamma-ray bursts (GRBs). Constraints can be derived from multiple directions, and we use three distinct strands; (i) direct observations of GRBs and their host galaxies, (ii) parameters derived from modelling, both via population synthesis and direct numerical simulation and (iii) our understanding of plausible analog progenitor systems observed in the local Universe. From these joint constraints, we describe the likely routes that can drive
\end{abstract}

\footnotetext{
A. Levan

a.j.levan@warwick.ac.uk

P. Crowther

Paul.crowther@sheffield.ac.uk

R. de Grijs

grijs@pku.edu.cn

N. Langer

nlanger@astro.uni-bonn.de

D. $\mathrm{Xu}$

dxu@nao.cas.cn

S.-C. Yoon

yoon@astro.snu.ac.kr

1 Department of Physics, University of Warwick, Coventry, CV4 7AL, UK

2 Department of Physics and Astronomy, University of Sheffield, Sheffield S3 7RH, UK

3 Kavli Institute for Astronomy \& Astrophysics and Department of Astronomy, Peking University, Yi He Yuan Lu 5, Hai Dian District, Beijing 100871, China

4 International Space Science Institute-Beijing, 1 Nanertiao, Zhongguancun, Hai Dian District, Beijing 100190, China

5 Argelander-Institut für Astronomie der Universität Bonn, Auf dem Hügel 71, 53121, Bonn, Germany

6 Dark Cosmology Centre, Niels-Bohr-Institute, University of Copenhagen, Juliane Maries Vej 30, 2100 Copenhagen, Denmark

7 National Astronomical Observatories, Chinese Academy of Sciences, Beijing 100012, China
} 
massive stars to the creation of long GRBs, and our best estimates of the scenarios that can create compact object binaries which will ultimately form short GRBs, as well as the associated rates of both long and short GRBs. We further discuss how different the progenitors may be in the case of black hole engine or millisecond-magnetar models for the production of GRBs, and how central engines may provide a unifying theme between many classes of extremely luminous transient, from luminous and super-luminous supernovae to long and short GRBs.

Keywords Gamma-ray burst: general · Supernovae: general

\section{Introduction}

Since their discovery in the late 1960s (Klebesadel et al. 1973), unveiling the origin of gamma-ray bursts (GRBs) has been a central goal of contemporary astrophysics. While at one point the number of proposed models was only modestly smaller than the number of detected GRBs (e.g. Nemiroff 1994), ${ }^{1}$ over the past $\sim 20$ years we have finally narrowed down this progenitor list. The step change in our ability to study GRBs arose from the discovery of afterglow emission in 1997-these panchromatic afterglows (Costa et al. 1997; Frail et al. 1997; van Paradijs et al. 1997) precisely pinpointed GRBs on the sky, enabling their cosmological origin to be secured via observations of both afterglows and host galaxies. It is now clear that GRBs are exceptionally luminous cosmological explosions, with energies (if considered isotropic) of up to $10^{54} \mathrm{erg}$ (e.g. Maselli et al. 2014; Perley et al. 2014), and redshifts ranging from $z=0.0085$ (35 Mpc) to $z>8$ (Tanvir et al. 2009), possibly $z>9$ (Cucchiara et al. 2011).

It became apparent in the early years of GRB observation that the distribution of durations was not a smooth single population, but consisted of at least two peaks (e.g. Mazets et al. 1981, 1982). This difference, secured by observations with BATSE in the 1990s (Kouveliotou et al. 1993), led to the identification of short and long duration GRBs, one with a typical duration (normally defined as $t_{90}$, the time over which $90 \%$ of the total energy release in $\gamma$-rays is recorded) of around $1 \mathrm{~s}$, and the other with a characteristic duration of about a minute. While further observations have identified additional possible sub-classes (see Fig. 1) at low luminosity (Soderberg et al. 2004, 2006; Liang et al. 2007a), or at intermediate (Mukherjee et al. 1998; de Ugarte Postigo et al. 2011) or ultra-long duration (Levan et al. 2011, 2014a), it is the short and long GRBs that make up the vast majority of the observed GRB population, ${ }^{2}$ and whose origin has been most intensely sought.

\footnotetext{
${ }^{1}$ A time of launch of the Compton Gamma-Ray observatory (GCRO) and its BATSE instrument there were $\sim 800$ GRBs observed from a variety of missions, while approximately 110 models had been proposed. Interestingly, despite this progress, the now favoured collapsar model for long GRBs was not on that list.

${ }^{2}$ The apparent distribution of GRB luminosities is an excellent example of Malmquist bias, where the brightest events are visible over a much larger volume. Hence, while the observed population is dominated by high luminosity events, the volumetric rates are dominated by much lower luminosity systems that generally escape detection.
}

8 Key Laboratory of Space Astronomy and Technology, National Astronomical Observatories, Chinese Academy of Sciences, 20A Datun Road, Beijing 100012, China

9 Department of Physics and Astronomy, Seoul National University, Gwanak-ro 1, Gwanak-gu, Seoul 151-742, Republic of Korea 


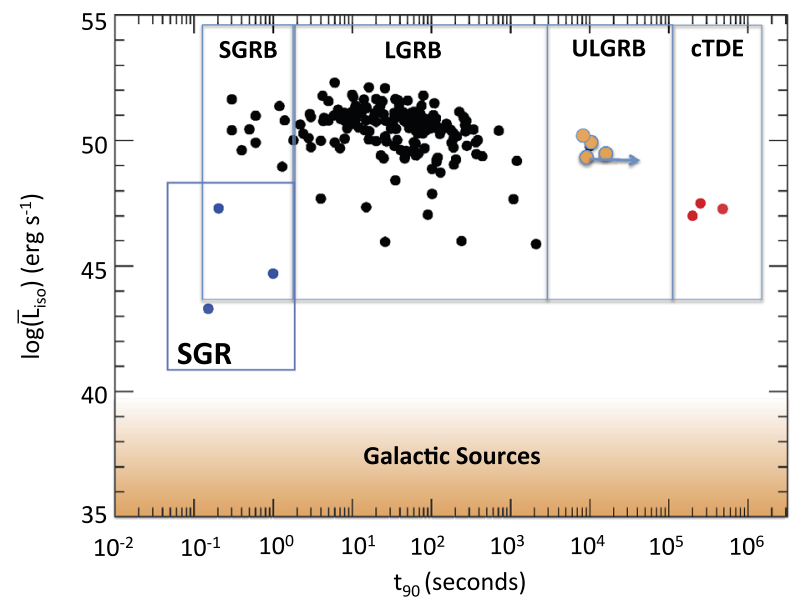

Fig. 1 High energy phase space for gamma-ray bursts, adapted from Levan et al. (2014a). The duration of the bursts is shown, compared to their mean luminosity over that duration. This demonstrates that the majority of observed bursts arise from the long GRB (LGRB) or short GRB (SGRB) population, while outliers are clearly present both at extreme durations, visible as the ultra-long GRBs (ULGRBs) and candidate tidal disruption events (TDEs) and at low luminosities. Indeed, it is the low luminosity long GRBs that provide the best studied associated supernovae. A group of the short GRBs are also likely to arise from giant flares from soft gamma-repeaters (SGRs) in external galaxies (e.g. Palmer et al. 2005; Hurley et al. 2005; Tanvir et al. 2005; Levan et al. 2008), although these have yet to be firmly identified

In this review, we discuss progress towards GRB progenitors that can be made from three distinct strands. The first is direct observations of the GRBs and the host galaxies themselves. From these observations, we can determine the nature of any additional sources of energy in the GRB, be they supernovae (SNe) signatures in long GRBs (e.g. Hjorth et al. $2003)$, or radioactively powered kilonovae ( $\mathrm{KNe}$ ) created by nucleosynthesis in the neutronrich disc or ejecta formed in short GRBs (Barnes and Kasen 2013; Berger et al. 2013; Tanvir et al. 2013). These additional sources are frequently observed as late photometric bumps, interrupting the smooth decay of the afterglow light. Their study enables information about the energy and chemical make-up of the GRB explosion to be extracted, and has been very important in pinpointing GRBs progenitors. Studies of the afterglow light also provide the potential to measure beaming angles in GRBs, and hence to convert the observed rate of GRBs to the volumetric rate of GRB-like explosions. Finally, studies of the host galaxies themselves provide information about the stellar population from which the GRB is born, and the dynamics of the progenitors. Taken together these observations provide a significant, but still incomplete view of the stellar systems that create GRBs.

The second strand of our consideration comes from theoretical modelling of both GRB progenitors, and the pathways that lead to their creation. The observed GRB energetics, and the presence of photons from GRBs well above the pair production limit, directly implies relativistic outflows (e.g. Cavallo and Rees 1978), which must somehow pierce their progenitors. This presents immediate and significant constraints on the nature of GRB central engines that must be able to release a significant fraction of a solar rest-mass rapidly, and into a baryon-free environment (the presence of baryons would entrain any ejecta, and make achieving relativistic velocity extremely difficult e.g. Lei et al. 2013). In assessing the progenitors of GRBs from a theoretical perspective it is necessary to both model the details of the proposed progenitor (for example its rotation, the mass of the compact remnant formed, 
the baryon loading in its immediate environment etc.) and also understand the routes to obtaining these progenitors. Through this route, it is possible to determine both the types of star that might create GRBs, and whether these systems are born at a necessary rate to match the observed GRB population.

The third and final element of this work, and one which is often overlooked is to consider how local populations can inform our studies. Long GRBs are created from massive, and most likely rapidly rotating stars, and there is increasing evidence that these are drawn from low, but not excessively low metallicity (e.g. Fruchter et al. 2006; Graham and Fruchter 2013; Krühler et al. 2015). Indeed, most estimates have a rapid drop-off in the GRB rate somewhere between solar (Krühler et al. 2015; Perley et al. 2015) and 1/3 solar (Graham and Schady 2015b) — in other words, between the Milky Way (roughly solar) and the Small Magellanic Cloud (approximately 1/5th solar). In that sense, studies of the massive stellar populations within the Local Group could place strong constraints on evolutionary pathways that are viable based on star formation in differing environments. Similarly, for short GRBs, the local population of compact binaries, both in the field and clusters can be used as a route to informing the rate and pathways to their production, although again this is a challenging prospect. Such observations are likely incomplete, for example, most NS-NS binaries are found via the radio emission from a spun-up (recycled) pulsar, and there is a clear observational bias against binaries that merge very quickly after their initial formation (i.e. they have already merged and cannot be observed). However, despite these issues, local populations can provide unique diagnostics and constraints and may be able to directly identify plausible GRB progenitors.

By tackling the issue of GRB progenitors from these three routes it is possible to begin to constrain not only the basic properties of the progenitors, but also details about their likely rate, the necessary environmental conditions for their production, the possible presence of their remnants in the local Universe, or the likelihood that any stars identified today may ultimately be GRB progenitors. It is also vital if we wish to place GRBs among the full range of transients produced in the deaths of stars and to understand the links between them. Perhaps most import here are the links between GRBs and super-luminous supernovae (SLSNe, e.g. Gal-Yam 2012). These SNe peak a factor of 100 brighter than most SNe, but interestingly a rather similar set of models are invoked to explain their origin (Gal-Yam et al. 2009; Kasen and Bildsten 2010), and intriguing similarities are present in their environments (Lunnan et al. 2014, 2015; Leloudas et al. 2015; Angus et al. 2016). All of this work has substantial implications in its own right but is also a necessary first step if we hope to use GRBs as increasingly precise cosmological tools, for mapping the history of star formation, the build up of metals, or even as signatures of the collapse of the first stars.

\section{What Do Observations Tell Us About Long GRB Progenitors?}

Progress towards understanding the nature of long-GRB progenitors has been one of the major success stories of the field and followed rapidly after the first precise locations became available. It is now clear that at least the majority of long GRBs arise from the core collapse of massive stars and are associated with hydrogen poor, high-velocity type Ic supernovae (e.g. Hjorth et al. 2003; Cano 2013). However, beyond this there remain central questions about the progenitors that have yet to be answered; just how massive are the stars creating GRBs, and are they classical Wolf-Rayet stars or something more exotic? Are binary channels important? What is the role of metallicity in creating GRB progenitors and what 
does this mean about their utility as cosmic probes? Exactly what central engines are created? Are the systems creating the long GRBs similar to those that are seen in the brightest supernovae?

\subsection{Building the GRB-SNe Connection}

The discovery of the extremely unusual, low luminosity GRB 980425 associated with a broad line type Ic supernova (SN 1998bw) marked the first strong hints as to the progenitors (Galama et al. 2000), although the total energy (if assumed isotropically released) of GRB 980425 was 4-6 orders of magnitude lower than seen in other GRBs, and it had little afterglow (AG) emission (Kouveliotou et al. 2004). Indeed, while GRB 980425 was amongst the first handful of events to be identified, it remains the closest known event of more than 1000 bursts with afterglows to date.

Despite these disparate observational properties, searches for similar supernova signatures in more distant GRBs gradually began, and possible examples were soon found as photometric bumps, causing a re-brightening of the optical counterpart on timescales of 2030 days after the burst (e.g. Bloom et al. 1999). These photometric bumps apparently had broadly similar peak luminosities to SN 1998bw and importantly were a factor ten or more brighter than the mean peak luminosity of core-collapse SNe. A number of examples were found in the following years, predominantly in the GRB population observed at relatively low redshift $(z<0.5)$ (e.g. Price et al. 2002, 2003a; Garnavich et al. 2003; Bersier et al. 2006), although in some cases with HST and 8-m telescopes at somewhat larger distances $(z<1)$ (Masetti et al. 2003; Price et al. 2003b), and in a few cases inferred from bumps in the absence of a redshift (Bloom et al. 1999; Gorosabel et al. 2005; Levan et al. 2005). These observations gradually built a consensus that some form of supernova was present, in at least the majority of long GRBs. However, detailed spectroscopic study, necessary, for example, to identify if hydrogen was present, remained challenging, as the redshifts to the majority of GRBs detected at the time lay beyond the capabilities of available spectroscopy.

Unsurprisingly, the search was on for a low-redshift, but intrinsically energetic GRB that would enable a direct comparison with the local, low-energy GRB 980425. Such an opportunity was presented by GRB 030329 , at $z=0.17$ a redshift at which a supernova signature could be readily visible (SN2003dh). The light curve of GRB 030329 itself was extremely complex (Lipkin et al. 2004), consisting of many re-brightenings due to ongoing energy injection (e.g. Price et al. 2003c; Willingale et al. 2004). However, despite this, the optical light clearly transitioned from the featureless GRB-afterglow power-law into a spectrum with marked similarities to that of SN 1998bw (Hjorth et al. 2003; Stanek et al. 2003), cementing the association of GRBs with broad-lined SN Ic.

\subsection{Distribution of Supernova Bulk Properties}

A large sample of GRB-SNe or candidate GRB-SNe has now been accrued. The majority of these arise from photometric bumps in the late time light curve, but an increasing number also have direct spectroscopic detections of the $\mathrm{SNe}$ in question, although often through noisy spectra at a single epoch. ${ }^{3}$ Inevitably this means that the quality of the spectra, and

\footnotetext{
${ }^{3}$ The GRB/SNe pairs with some spectroscopic evidence include GRB 980425/SN 1998bw, GRB 021211/SN 2002lt, GRB 030329/SN2003dh, GRB031203/SN20031w, GRB 060218/ SN2006aj, GRB100316D/SN2010bh, GRB 10129B/SN2010ma, GRB 111209A/SN 2011kl, GRB120422A/SN2012bz, GRB 130427A/SN2013cq, GRB 130215A/SN2013ez, GRB 130702A/ SN 2013dx, GRB 140606B/iPTF14bfu.
} 
decisions of different authors about the merits of including a given burst vary. However, excepting GRB 111209A/SN 2011kl (Levan et al. 2014a; Greiner et al. 2015a) the spectra of all of these events bear marked similarities to that of SN 1998bw, showing broad lines consistent with high-velocity expansion $>20,000 \mathrm{~km} \mathrm{~s}^{-1}$, and no sign of hydrogen or helium emission features. All of these SNe are therefore spectroscopically classified as broad-lined type Ic supernovae (SN Ic-BL).

The task of obtaining broad-band spectral shapes and peak luminosities from light curves of these $\mathrm{SNe}$ is made challenging by their large luminosity distances, by the contribution of the host galaxy, and by the afterglow contribution. Indeed, at any given time the observed flux depends on the contribution of all three, e.g.

$$
F_{\mathrm{obs}}(\nu, t)=F_{\mathrm{AG}}(\nu, t)+F_{\mathrm{SN}}(\nu, t)+F_{\mathrm{host}}(\nu) .
$$

$F_{\text {host }}$ can in principle be obtained from late-time observations and subtraction from early data, although in practice this is often complicated by slightly different filters or instrument combinations, as well as the difficulties introduced by matching ground-based seeing. A typical GRB host is of comparable (or perhaps slightly lower) luminosity than its SNe (Savaglio et al. 2009; Svensson et al. 2010; Perley et al. 2015) and so can create a significant uncertainty, especially for higher redshift bursts, and even more so when spectroscopy is used (since the spectroscopy is inevitably noisier than imaging). The afterglow can normally be treated as a power-law in both frequency (or wavelength) and time e.g.,

$$
F_{\mathrm{AG}}(v, t)=t^{-\alpha} v^{-\beta} \text {. }
$$

Measurements of the afterglow can be complicated by unseen temporal breaks (e.g. the jet-break due to lateral spreading of the GRB-jet, which typically steepens $\alpha$ to $\sim 2$ (e.g. Rhoads 1999)) or spectral breaks (e.g. the cooling break, which imparts $\Delta \beta=0.5$ (e.g. Sari et al. 1998)).

Given the spectral similarities to SN 1998bw, a common approach is to assume that the light curve of the SN evolves in time, $t$, like that of SN 1998bw but modified by a stretch parameter $s$, and luminosity scaling, ${ }^{4} h$.

$$
F_{\mathrm{SN}}=h F_{98 \mathrm{bw}}(t / s),
$$

where $F_{98 \mathrm{bw}}$ is the specific flux of SN 1998bw as observed at the same rest-frame wavelength and time as that of the burst under consideration (i.e. taking into account a k-correction, and cosmological time dilation).

One can fit for these things simultaneously in well-sampled data (e.g. Zeh et al. 2004; Kann et al. 2010; Cano 2013; Cano et al. 2014), although degeneracies can exist (e.g. between $h$ and $\alpha$ ), and it is also common to attempt to extrapolate the afterglow contribution, either from earlier observations, or from data in the UV or IR where the SNe contribution is small (e.g. Levan et al. 2014b).

In practice, this is a relatively crude approach, since the SNe light curve may not be a good approximation to a stretched and scaled SN 1998bw. However, in many cases it appears to work well, at least given the data available. More recent attempts have moved beyond simple scaling factors and have attempted to infer bolometric properties, either from the scaled SN 1998bw light curves (e.g. Cano 2013) or using other SNe as templates, enabling single colours to be used for bolometric corrections (e.g. Lyman et al. 2014a, 2016a).

\footnotetext{
${ }^{4}$ Here we use $h$ to determine the luminosity scaling to avoid confusion with the k-correction, however in other works it is common to see the luminosity scaling expressed as $k$.
} 


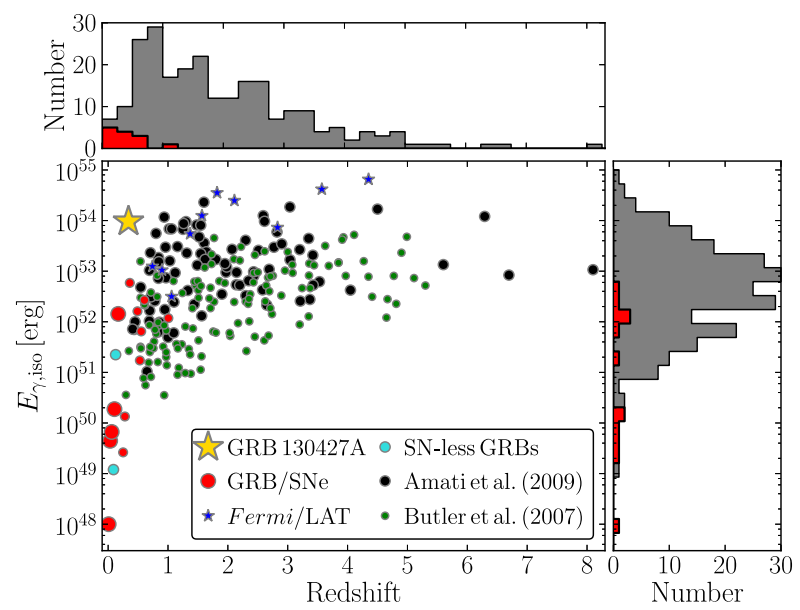

Fig. 2 The distribution of isotropic equivalent energy with redshift for long GRBs from Xu et al. (2013), with the background points determined by Butler et al. (2007) and Amati et al. (2009). Those with spectroscopic detections of a supernova are marked in red. As can be seen these are typically low redshift events with a low luminosity (these bursts are likely intrinsically common, but only visible at low-z). Only a handful of events are typical of the more energetic GRBs that make up the bulk of the observed GRB population, with only GRB 130427A lying at the brightest end of the distribution

One thing which is clearly apparent in these observations is that the classical long duration, highly energetic GRBs that comprise the bulk of the observed GRB population are highly under-represented in the GRB-SNe sample (Fig. 2). In itself, this is not surprising, since $\mathrm{SNe}$ are most readily seen in low redshift examples, and when not outshone by the afterglow (whose luminosity broadly scales with that of the $\gamma$-rays, (e.g. Nysewander et al. 2009a; Gehrels et al. 2008)). Indeed, many of the GRBs arise from a potentially distinct population of low-luminosity GRB (LLGRB), while even the Rosetta Stone of GRB 030329/SN 2003dh is of rather intermediate luminosity. Only GRB 130427A/SN 2013cq appears to arise from a highly luminous GRB (Xu et al. 2013; Levan et al. 2014b; Melandri et al. 2014).

While this may not be problematic, it is notable that in some cases these local, low luminosity bursts appear very different from their higher energy cousins. In particular, some are extremely long and dominated by thermal emission (Campana et al. 2006; Starling et al. 2011), features often not seen in other GRBs. It this sense it may be that they are not indicative of GRB-SNe in general, although it may also be that similar components in more distant, luminous GRBs escape detection, and some thermal components have been found in careful searches (Starling et al. 2012; Sparre and Starling 2012), perhaps suggesting similarities. Indeed, the strongest link of the similarities between these very different energies of high energy transient is actually the properties of their supernovae, which are sufficiently similar that it is likely they all arise from the same physical mechanism.

\subsection{Test-Bed Examples}

An alternative to the large scale samples accrued with data of variable quality is to attempt detailed modelling of well-studied examples, where time series spectra and excellent photometric coverage is available. In these cases it is possible to go beyond simple analytical fits to light curves, or snapshot velocities from spectroscopy, and build detailed models of the 
explosions of a range of stars that map the nickel releases, velocities, and kinetic energies, providing high-quality spectrophotometric predictions that can be compared with observations. The principle here is to conduct detailed spectral synthesis, in which energy injection from the core is coupled with radiation transport to predict the observed spectrum at given epochs as a function of the various input parameters. In principle, these models can also include detailed geometry, for example, ejecta which includes anisotropies in order to match both the rise/decay times of the SNe. This avoids the need to use scaling relations to obtain $\mathrm{SNe}$ properties and instead obtains them from ab initio approaches. It has been successful in several GRBs, including GRB 980425/SN 1998bw (e.g. Höflich et al. 1999; Woosley et al. 1999; Maeda et al. 2002), GRB 030329/SN 2003dh (e.g. Deng et al. 2005) and GRB 060218/SN 2006aj (Pian et al. 2006b; Mazzali et al. 2006). Results from such approaches are reassuringly often similar to those from light curves alone in terms of bulk properties (e.g. nickel mass, Cano 2013), although also provide evidence at times for anisotropies in the explosion, and stronger constraints on pre-explosion core mass and ejecta mass. However, the observational requirements for such work are frequently extreme, and so for the majority of GRBs such detailed information is either too expensive or impossible to obtain via current instrumentation.

\subsection{Pushing the Boundaries: From no Supernovae to the Most Luminous Supernovae}

While broad-lined type Ic supernovae have been discovered in the vast majority of cases where such a search was plausible there are a handful of cases where such searches have been unsuccessful. In particular, two local long GRBs, 060505 and 060614 (at $z=0.09$ and $z=0.125$ ) lie at redshifts where SNe similar to SN 1998bw should be readily identified, with peak apparent magnitudes in the range $18.5<m_{V}<19.5$. However, deep searches failed to identify any such signatures to limits not only much fainter than GRB-SNe but also to other core-collapse events. Indeed, any SNe in these cases must have been a factor $>100$ fainter than SN 1998bw (Della Valle et al. 2006; Fynbo et al. 2006; Gal-Yam et al. 2006; Gehrels et al. 2006). It has been noted that GRB 060505 was of relatively short duration $\sim 4$ s, while GRB 060614 has subsequently been suggested to be an example of a short burst with extended, softer emission (Gehrels et al. 2006). This scenario seems likely, since further examples of SN-less, but unambiguously long GRBs have not been uncovered. However, other suggestions, for example, that these GRBs may arise in cases where no outward supernova shock is launched and the star collapses directly to a black hole (Fynbo et al. 2006; Fryer et al. 2007) have also been made. This may be particularly relevant given the apparent absence of very massive progenitors to local SN II-P (Smartt et al. 2009), which has led to renewed interest in the prospect of disappearing stars (Kochanek et al. 2008; Kochanek 2014), with recent surveys beginning to find candidate examples (Reynolds et al. 2015). Surprisingly, despite the decade since these discoveries, further examples have not been found, and so their nature remains mysterious.

At the other end of the scale, the recent discovery of an SN-GRB (SN 2011kl/GRB 111209A) which was a magnitude more luminous than SN 1998bw (Greiner et al. 2015a; Kann et al. 2016), challenges the picture of SN homogeneity that has been emerging from previous observations. This SN was found in an ultralong GRB with a duration of $>10,000 \mathrm{~s}$ (Gendre et al. 2013; Levan et al. 2014a), and in addition to being more luminous than most GRB-SNe was also spectrally very different. In particular, while it also appears to belong to the SN Ic population, it was far bluer and more UV luminous than other GRB-SNe, and its spectrum bore a strong resemblance to the spectra of type I SLSNe, which also show weak 
absorption lines on an extremely blue continuum (e.g. Mazzali et al. 2016). Other ULGRBs do not show such strong apparent $\mathrm{SNe}$, and so it remains unclear if this burst is a unique and unusual object, or if it in fact represents a broader range of SNe properties that should be considered, perhaps from cases where the central engine of the GRB begins to impact the supernova itself, as is likely the case for SLSNe (e.g. Cano et al. 2016).

\subsection{Large Scale Environments}

Additional constraints on the progenitors of long GRBs can be obtained from both their large scale and small scale environments. The metallicities of their host galaxies are of particular interest (in practice, the metallicities of the small scale environments are also of interest, but are not readily accessible with current technology, since one arcsecond seeing typically corresponds to several kiloparsecs at the redshift of a typical long GRB). Such constraints can either come from direct spectroscopic observations, or via inferences using the well-known correlation between mass (or luminosity) and metallicity. GRBs can provide exceptional diagnostics of metallicity at high redshift via studies of UV absorption lines in concert with the direct detection of Ly $\alpha$ (e.g. Fynbo et al. 2009). However, this is predominantly for higher redshift bursts, since Ly $\alpha$ does not enter the optical window until $z>2$, and a measurement of the hydrogen column density $N_{\mathrm{H}}$ along the line of sight is necessary to obtain the ratio of a given element to hydrogen (e.g. $\mathrm{O} / \mathrm{H}, \mathrm{Fe} / \mathrm{H}$ ). For this reason the metallicities of the host galaxies where the GRB-SNe connection has been determined are predominantly obtained either from emission line diagnostics such as, $R_{23}=([\mathrm{OII}](3727 \AA)+[\mathrm{OIII}](4959+$ $5007 \AA)) / \mathrm{H} \beta$ or $\mathrm{N} 2=\log [\mathrm{NII}] / \mathrm{H} \alpha$, or indeed more complex approaches to line ratios that attempt to remove degeneracies that exist is the more simple examples (e.g. Dopita et al. 2016). This provides some insight into the chemical state of the gas-phase in these galaxies, although several important caveats should be noted in the use of these metallicities as a direct insight into GRB progenitors. Firstly, they are not a measurement of the metallicity of the progenitor star, and indeed, even if they are an accurate measurement of the oxygen metallicity of the star, this is still some way from the $[\mathrm{Fe} / \mathrm{H}]$ ratios that are more commonly used in distinguishing different stellar evolution pathways. Secondly, significant metallicity gradients can exist within galaxies, and so the use of global proxies essentially derives central metallicities (i.e. those of the brightest regions), which may, or may not be indicative of the regions hosting a given transient event. Thirdly, in some cases, the GRB may be associated with a satellite galaxy of the presumed host. For example, Kelly et al. (2013) show that GRB 130702A (at $z=0.145$ ), lies in a satellite galaxy at a significant offset (18 kpc). However, in other cases it is likely that more proximate galaxy/satellite pairs are not adequately resolved, at least by ground-based imaging, leading to the misidentification of the host. In extremum, there can even be chance alignment in which a background galaxy aligns with a foreground system leading not only to the misidentification of the precise host galaxy but the incorrect determination of the redshift. This has recently been demonstrated for an extremely well studied optically dark (i.e. no optical afterglow) GRB 080219B, which, rather than lying at $z=0.41$ is in fact in a background galaxy at $z=1.96$ (Perley et al. 2016). In these cases, the use of metallicity measurements inferred from the "apparent" host is clearly incorrect, and potentially problematic. For example, the case of GRB 080219B has been used to argue for the presence of GRBs in high metallicity environments, but in practice provides no such evidence since this metallicity is simply of the foreground system.

The range of metallicities seen in long GRB host galaxies (as well as short GRBs and luminous $\mathrm{SNe}$ ) is shown in Fig. 3. It is clear that GRBs favour a lower metallicity than is typical in the local Universe, although given the redshift range considered this is not surprising. Interestingly, the metallicities of long GRB hosts at large appear systematically higher 
Fig. 3 Host galaxy metallicities for long GRBs from Krühler et al. (2015), short GRBs (from Berger 2009) and SLSNe (from Leloudas et al. 2015). It is striking that the metallicities of LGRBs appear significantly lower than for the SGRBs, or indeed the general galaxy population (Graham and Fruchter 2013). However, those of the GRBs with apparent SNe appear to be somewhat lower still, and are very similar to those observed in SLSNe host galaxies

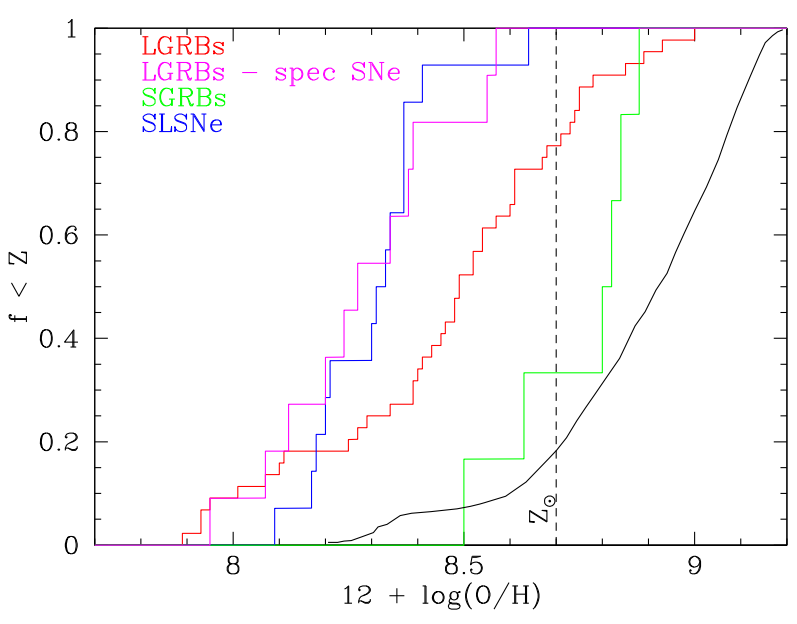

than those of the examples in which a spectroscopic signature of the SNe has been seen, with the median metallicity of the GRB-SNe sample being $0.2-0.3$ dex lower. This may well be due to a bias against dusty systems, since obscured star formation is typically more metal rich, and it is clear that dusty GRBs arise in more luminous, and likely metal-rich host galaxies. In any case, it also interesting to note, that when considering only the GRBs in which spectroscopic SNe signatures are seen there is an apparent similarity between their metallicity distribution and those of SLSNe. The degree to which these differences arise due to observational selection effects, or alternatively due to genuine astrophysical differences is of significant interest for further study. It may reflect genuine difference between populations, or perhaps that even in the relatively low redshift regime there may be significant selection effects (e.g. Japelj et al. 2016).

\subsection{Small Scale Environments}

The large scale environments can provide information about the chemical state of GRB hosts that is not currently available at smaller scales, due to both large luminosity distance, and the inability of large aperture telescopes to probe with sufficiently high resolution. However, star formation takes place on small spatial scales, from less than a parsec to tens of parsecs depending on the mode and intensity of star formation (e.g. Portegies Zwart et al. 2010). It is these small scale environments that in practice carry most information about the GRB progenitor itself, free from dilution from the light of the remaining galaxy. At a typical GRB redshift, the $H S T$ resolution $(0.1$ arcsec) still corresponds to physical scales of several hundred parsecs, and so while we are able to track the stellar populations in the regions of the GRBs, isolating the stellar population responsible for the GRB remains challenging. Two approaches can be considered when studying GRB locations. The first is simply to identify the GRB location relative to its host galaxy by measuring its offset, either relative to the galaxy host (e.g. Bloom et al. 2002), or perhaps to local regions of intense star formation, if such resolution is available (e.g. Hammer et al. 2006). The alternative is to try to study the population under the burst, despite the poor spatial resolution. This may be highly diagnostic for long GRBs, since the young massive stars are by far the most luminous in the host galaxy, especially at UV wavelengths (Fruchter et al. 2006). Indeed, at the typical redshifts of many GRBs rest frame UV light is redshifted into the optical window, making sensitive high-resolution imagery with HST an ideal route to characterising the immediate massive 
star populations surrounding GRBs. In addition, these hot massive stars also excite the gas phase of the interstellar medium, making narrow-band observations a sensitive probe of the young massive star population (James and Anderson 2006).

Such an approach has been taken by various groups studying both supernovae and GRBs. Fruchter et al. (2006) consider the total fraction of the galaxy light in pixels of lower surface brightness than the pixel containing the GRB or SNe, the so-called $F_{\text {light }}$ parameter. They show that GRBs are highly concentrated on the light of their host galaxy, while core collapse $\mathrm{SNe}$ broadly trace the distribution of rest-frame UV-light. In other words, the probability of a SN occurring in a given pixel is approximately proportional to the brightness of that pixel, in contrast, the probability of a GRB occurring in a given pixel is proportional to the brightness of that pixel squared. ${ }^{5}$ Interestingly, further studies of local SNe show that a similar effect can be seen in the distribution of hydrogen-rich SNe II and hydrogen/helium poor SNe Ic (James and Anderson 2006; Kelly et al. 2008; Anderson et al. 2012). This is likely understood because of the strong correlation between stellar mass and luminosity. In the low mass range $\left(10-20 \mathrm{M}_{\odot}\right) L \sim M^{3}$, while for higher mass stars $\left(50-100 \mathrm{M}_{\odot}\right)$ $L \sim M^{2}$ (Yusof et al. 2013) such that the most massive stars dominate the UV budget. In this sense, if core collapse $\mathrm{SNe}$ arise from essentially every star with an initial mass $>8 \mathrm{M}_{\odot}$, then GRBs must arise from stars significantly more massive than this. Studies based on the expected distribution of stars in galaxies (Raskin et al. 2008), and on young clusters in nearby galaxies (Larsson et al. 2007) provide a consistent picture in which GRB progenitors have initial masses of $>40 \mathrm{M}_{\odot}$, representing one of the few ways in which the masses of GRB progenitors can be ascertained.

\subsection{Summary: LGRB Progenitors}

The emerging picture for long GRB progenitors appears clear. A long GRB progenitor is a massive star, typically born at sub-solar metallicity, in which the hydrogen envelope is lost/burned prior to its explosion as a broad-lined type Ic supernova. There is some observational evidence that suggests these stars are very massive, for example, the large Nickel yields required to achieve absolute magnitudes a factor 10 brighter than $\mathrm{SNe}$ Ic (e.g. Mazzali et al. 2007a), or the location of the bursts on the brightest regions of their hosts (Fruchter et al. 2006; Svensson et al. 2010) could both be interpreted as signatures of very massive stars, perhaps with an initial mass at the zero-age main sequence (ZAMS) of $M_{\text {ZAMS }}>40 \mathrm{M}_{\odot}$. However, the locations of long GRBs are similar to those of the bulk SNe Ic population (Kelly et al. 2008), most of which can be explained by the explosion of initially far less massive stars (e.g. Mazzali et al. 2007b,c), and more complex models, involving the impact of chemically homogeneous evolution (e.g. Szécsi et al. 2015), or binaries (e.g. Stanway et al. 2016) on the observed environments may be necessary to provide tighter constraints on the progenitor mass, as well as on the progenitor rotation. These scenarios are not currently well constrained observationally, and theoretical progress is discussed in Sect. 5. None-the-less, the progress which has been made towards the progenitors of long GRBs has been remarkable, and while there remain many open questions about the details of long GRB progenitors, it is fair to say that barring a few special examples, we now know what forms the vast majority of the long GRB population.

\footnotetext{
${ }^{5}$ The squared exponent here is approximate, and does not carry any specific meaning.
} 


\section{Observational Constraints on Short GRB Progenitors}

\subsection{Defining Short GRBs}

Short GRBs are traditionally defined as those with durations of $t_{90}<2 \mathrm{~s}$. Observations with Konus and BATSE suggested they form a separate population from the long GRBs, and so may well also have different progenitors (Mazets et al. 1981, 1982; Kouveliotou et al. 1993). Following the identification of supernovae signatures in the afterglows of long GRBs, the favoured model for short bursts rapidly became the merger of two compact objects (neutron star-neutron star or neutron star-black hole), a model which had previously been popular for the long GRB population (e.g. Narayan et al. 1992). The reason for this was twofold; both that the rapid time scale for the merger, combined with the clean environment (compared to e.g a collapsing massive star) meant that short durations were more naturally expected in merger scenarios. The second was the rather more speculative assertion that if mergers did not produce the long bursts, they must either produce the short bursts, or no high energy transient at all. Given this historical approach, it is unsurprising that the observational history of short GRBs has largely been benchmarked against this expectation, and hence the discussion of short GRBs has been largely framed as though their progenitors are known, even prior to the accrual of the significant observational data that now supports a merger origin.

Short bursts now represent approximately $10 \%$ of the bursts observed by Swift, down somewhat from the $25 \%$ observed in the BATSE observations. It seems likely that a significant element of this is due to the differing spectral responses of the different instruments, with BAT operating in a rather softer 15-150 keV band compared to BATSE $(50->300 \mathrm{keV})$. This has led to some discussion of whether the duration split between the two populations is correctly set at 2 seconds. Indeed, the duration distribution of Swift GRBs is far less clearly bimodal than that for BATSE, and so one cannot directly distinguish a "cut-off" based on these observations alone. Several alternative attempts in the early years of Swift addressed the potential problem by suggesting the use of additional diagnostics to distinguish between different progenitor types (e.g. Levan et al. 2007; Zhang et al. 2009). Indeed, Zhang et al. (2009) argued for a distinction between Type I (compact object merger) and Type II (collapsar) events based on a decision tree of multiple observational properties. These additional constraints often used rather more complex properties (for example the nature of the host galaxy, or the presence/absence of a supernova component) and so also risked significant confirmation bias, in finding bursts which met the expectations based on only a handful of systems and omitting other, potentially valuable systems. Using theoretical considerations, largely based on the break-out time for a collapsar, combined with the different spectral responses of different observatories Bromberg et al. (2013b) have suggested that very different values for $T_{90}$ would be needed to identify the split between long and short GRBs for different missions. In particular, $2 \mathrm{~s}$ for BATSE and $0.7 \mathrm{~s}$ for Swift. ${ }^{6}$ The essence of the argument is that hardness is a more important distinction than duration at durations where there is likely to be significant overlap between the two populations. This is shown graphically in Fig. 4, where the Swift short GRBs can be seen to be typically much softer than those detected by BATSE. Applying a cut of this nature would remove some (although by no means all) of the best studied "short GRBs" from consideration, since they would then be more likely to be collapsars.

\footnotetext{
${ }^{6}$ At $50 \%$ probability. Since the standard assumption is to think of the duration distribution of long and short GRBs as Gaussians, there is inevitably overlap in the two populations.
} 


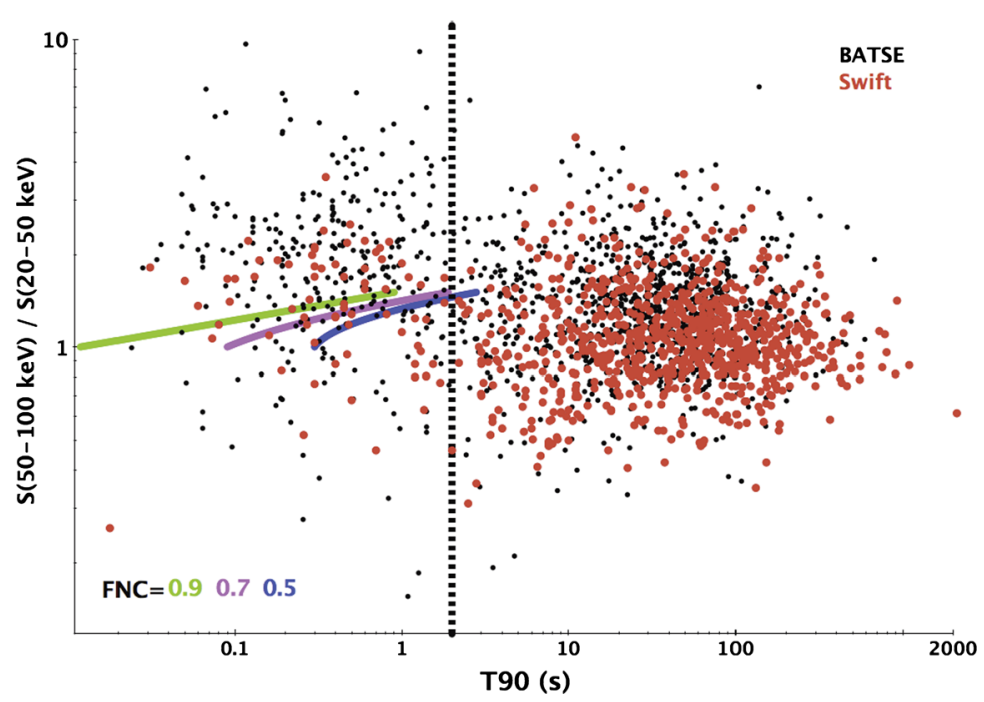

Fig. 4 The hardness-duration distribution of GRBs detected by Swift (red) and BATSE (black). The $x$-axis shows the duration over which $90 \%$ of the total fluence is recorded ( $T_{90}$, while the $y$-axis shows the $\gamma$-ray hardness, defined as the ratio of flux between two adjacent channels (the channels chosen are those used by BATSE). The Swift data have been placed on the same scale by extrapolating the prompt spectral fits (either as a power-law or cut-off power-law) to the appropriate bands. The dotted vertical line shows the notional distinction between short and long GRBs at two seconds, but there is clearly significant overlap. It is apparent from the BATSE distribution that short GRBs are on average harder than long GRBs. However, for Swift the two distributions look very similar. Given this the contribution of the long GRB population is larger for Swift bursts at durations of $<2 \mathrm{~s}$ than for BATSE. The lines suggested by Bromberg et al. (2013a) as distinguishing long and short GRBs at 50,70 and $90 \%$ confidence are also shown (where FNC is the fraction of non-collapsars), bursts below these lines are more likely to be collapsars from the short duration tail of the long GRB population. For Swift, only bursts above the $90 \%$ green line are highly likely to be genuine members of the short burst population

\subsection{Precise Locations from Afterglows}

While progress towards the origin of long GRBs proceeded at pace from the discovery of the first afterglows to the identification of broad-lined SNe-Ic, pinpointing the progenitors of the short GRBs remained much more challenging. Firstly, short GRBs are typically significantly fainter, or more precisely exhibit markedly lower fluence than long GRBs despite similar peak fluxes (a parameter which more accurately describes their detectability to many $\gamma$-ray observatories). Since broad correlations exist between the fluence of the prompt emission and the brightness of either the X-ray or optical afterglow (Gehrels et al. 2008; Nysewander et al. 2009b) it is perhaps unsurprising that the afterglows of short GRBs escaped detection while the afterglow revolution was transforming our knowledge of the long GRB phenomena. Indeed, while a handful of short GRBs were detected and reasonably localised during the long GRB afterglow revolution, no successful afterglow campaigns were made prior to the launch of Swift. Its ability to re-point its X-ray and UV-optical telescopes rapidly provided the first X-ray afterglow detection for GRB 050509B (Gehrels et al. 2005; Hjorth et al. 2005a; Bloom et al. 2006). Interestingly, the first optical afterglow detection actually came from a HETE-2 burst, GRB 050709 (Hjorth et al. 2005b; Fox et al. 2005), which with the detection of another HETE-2 burst in early 2006 (de Ugarte Postigo et al. 2006; Levan et al. 2006), perhaps suggested that previous searches had been rather unlucky, although 
doubtless the ability of Swift to rapidly repoint and detect their X-ray afterglows was also an important component in the discovery of their optical counterparts.

As with long GRBs, the discovery of afterglows to short GRBs has revolutionised their study. Perhaps the most important diagnostic enabled by an afterglow is a precise location on the sky, and hence the ability to study the galaxy population hosting short GRBs. The first short burst with an afterglow was GRB 050509B, and its location was striking, being offset $\sim 30 \mathrm{kpc}$ from an extremely massive galaxy in a merging cluster system at $z=0.225$ (Gehrels et al. 2005; Bloom et al. 2006). Deep searches for star formation in this host galaxy revealed no sign, and immediately suggest that some short GRBs arise from ancient populations. However, this burst was localised only by its X-ray afterglow, and the large resulting error box contained additional background galaxies, many of which had blue colours consistent with star formation. Hence, while the probability of chance alignment was low, the association did not clinch the origin of at least some short GRBs in ancient populations. The discovery of the optical afterglow of GRB 050724 in a clearly elliptical host galaxy further strengthened this argument (Berger et al. 2005), and while bursts in low star formation rate host galaxies are clearly in a minority, perhaps 10-20\% of short GRBs overall do arise from elliptical hosts (Fong et al. 2013). Equally, while the remaining 80-90\% of the host galaxies do show signs of significant star formation, they remain distinct from the hosts of the long bursts in terms of their stellar masses and star formation rates. While long GRBs show a strong preference for star-forming dwarfs, with low metallicities and high specific star formation rates (e.g. Fruchter et al. 2006; Savaglio et al. 2009; Svensson et al. 2010; Graham and Fruchter 2013), the short bursts are in rather more typical galaxies, with a range of metallicities and higher stellar masses, therefore they appear to sample the entirety of the galaxy population (Leibler and Berger 2010; Fong et al. 2013).

Beyond the nature of the galaxies themselves, there is significant information contained within the distribution of the short GRBs on their hosts. Again, the differences between long and short GRBs become apparent rapidly in this distribution. While long GRBs are highly concentrated on their host light (Bloom et al. 2002; Fruchter et al. 2006; Svensson et al. 2010, see Sect. 2.6) the short GRBs are frequently scattered on their host light, and at large projected radii, in some cases there is apparently no underlying host galaxy, despite relatively bright galaxies nearby in the field, suggesting that these progenitors have been kicked from their birth sites (Berger 2010; Tunnicliffe et al. 2014), although it remains plausible that some lie at much higher redshifts in galaxies too faint for current observations (Levan et al. 2006; Berger et al. 2007). Beyond this much broader distribution, those short GRBs that do lie on galaxies tend to appear on much fainter pixels than those in the long GRBs (Fong et al. 2010; Fong and Berger 2013), and in particular show essentially no association with the blue light of their host galaxies. Taken together these properties demonstrate that short GRB progenitors are frequently old, and more importantly are also kicked from their birthplaces. These are all consistent with the expectations of binary mergers, in which the neutron stars receive substantial space velocities (kicks) from a combination of natal kicks and binary mass loss (e.g. Arzoumanian et al. 2002).

\subsection{Kilonovae and Macronovae}

The large scale observations are clearly broadly in favour of a model in which short GRBs arise from compact object mergers. However, they are in themselves not a smoking gun of the merger itself. This situation is reminiscent of early studies of long GRBs in which the star-forming host galaxies offered evidence of a link to massive stars and supernovae, but did not conclusively prove it. Since afterglows are generated at large radii and are essentially 
a product of the interaction of an outgoing relativistic shock with an external medium, they themselves are not uniquely diagnostic of the progenitor. Hence, some additional signature that could in principle only arise in compact object mergers was necessary. The most likely scenario is to identify a faint radioactively powered transient, created by nucleosynthesis in the neutron-rich material available in a compact object merger containing a neutron star. This material may reside either in the accretion disc, or be ejected into tidal tails. None-theless it was recognised that for typical properties of a merger such a transient would yield a rapidly evolving, faint transient, significantly faster and fainter than normal core-collapse SNe (e.g. Li and Paczyński 1998). Like a supernova, these events would start faint and rise to peak on timescales of hours to days, and so would be seen as photometric bumps, interrupting the otherwise smooth decay of the afterglow. These events have had a range of names over the years, but those which are commonly used today are either kilonova (referring in essence to something about 1000 times brighter than a nova), or macronova. The precise properties of these transients were uncertain, and while some populations of faint, fast transients have been uncovered by recent synoptic sky surveys (e.g. Kasliwal et al. 2012; Foley et al. 2013), none have been interpreted as NS-NS or NS-BH mergers (although some have been suggested to arise from NS-white dwarf mergers, through channels similar to those creating NS-NS binaries (Metzger 2012; Lyman et al. 2014b, 2016b). Hence, there is little observational evidence as to the signatures that observers should search for. Indeed, early, deep observations of several short GRBs failed to uncover any sign of the moderate to late time "bumps" expected in this scenario (e.g. Hjorth et al. 2005a) and ruled out the most optimistic scenarios for these transients (Metzger and Berger 2012).

However, an important revelation came from detailed calculations conducted in 2013 (Barnes and Kasen 2013). It had long been recognised that NS-NS mergers were promising sites for r-process nucleosynthesis, and that NS-NS mergers (depending on the rates and individual r-process outputs) could be important, if not dominant sites for the creation of the heaviest elements (Rosswog et al. 1999, 2003). Indeed, a range of recent studies from the abundances of radioactive elements on the sea floor (Wallner et al. 2015) to in depth analysis of metal poor stars in the Milky Way (e.g. Macias and Ramirez-Ruiz 2016) suggest that the r-process abundances are not in an equilibrium that would be expected if they were regularly replenished with small amounts of additional material, as might be expected for core collapse supernovae. Instead, these analyses prefer a scenario in which rare events, with significantly more mass per event are dominant, favouring a merger origin. This nucleosynthesis should naturally power a luminous transient, but because of the dominance of r-process elements its evolution might be quite different to those previously assumed based on our studies of supernovae. Indeed, the opacities that were assumed in the earlier predictions were predominantly those of iron group elements which are dominant in normal $\mathrm{SNe}$. The impact of including new opacities for heavy elements, in particular, lanthanides, was profound. The heavy opacity extinguishes essentially all the optical light for external observers and means that earlier observations were, in essence, unconstraining of the nucleosynthesis taking place. These observations instead suggested that IR observations were the most promising route to the identification of a kilonova (Barnes and Kasen 2013).

This supposition was observationally tested with HST observations of the short GRB 130603B in June 2013. These observations took place in two bands, one in the optical and one in the IR, for a burst at $z=0.35$ (de Ugarte Postigo et al. 2014). Although only two epochs were obtained they successfully showed a fading IR source, while nothing was seen in the optical (Berger et al. 2013; Tanvir et al. 2013). A comparison with the IR afterglow decay suggested a magnitude much brighter than expected, and hence a re-brightening from 
Fig. 5 The light curve of the kilonova seen in GRB 130603B (updated from Tanvir et al. 2013). The points represent the X-ray (black) optical (blue) and IR (red) photometry of the afterglow, along with their expected decay. The shaded region is the expected kilonova range for $10^{-1}$ and $10^{-2} \mathrm{M}_{\odot}$ of material from Barnes and Kasen (2013). While the cyan line shows the very faint expected optical emission. The re-brightening in the IR is strongly suggestive of a kilonova, although it is also relevant to note that late time X-ray observations by Fong et al. (2014) also imply that the X-ray lies above expectations

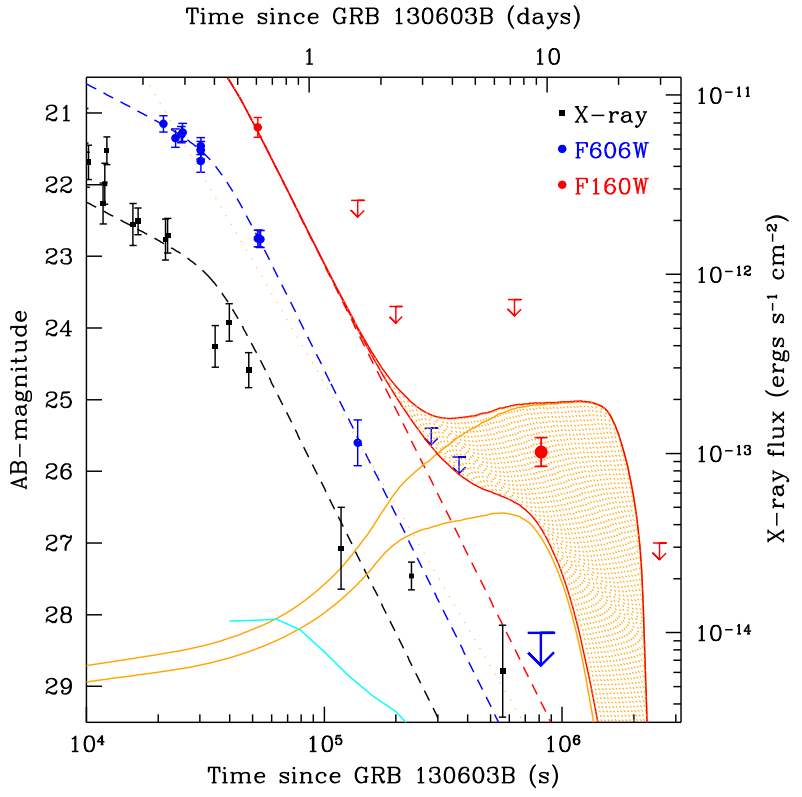

an associated kilonova (Fig. 5). The luminosity of the IR source was very similar to the prediction of kilonovae using the improved lanthanide opacities, and providing the first direct evidence for the origin of short GRBs in compact binary mergers.

Unfortunately, opportunities to further hone our understanding remain limited due to the rate of short GRBs at sufficiently low redshift, and so to date there are limited constraints on the true properties of this KNe. A detailed re-analysis of some archival short GRBs provides some evidence for similar components in their light curves (e.g. Jin et al. 2015), although other short GRBs show optical or X-ray bumps apparently not associated with the same physical process (Perley et al. 2009). Indeed, recent work, partly motivated by an apparent X-ray bump co-incident with the infrared kilonova in GRB 130603B (Fong et al. 2014) has focused on possible long-lived X-ray manifestations of mergers. In particular, in the form of scattered X-ray's from the central engine (either millisecond magnetar or black hole) (Kisaka et al. 2015), the absorption and re-radiation of X-rays to provide IR and X-ray signals (Kisaka et al. 2016), or direct emission of X-rays from the engine itself (Sun et al. 2016), perhaps creating isotropic X-ray emission that could be of some value in searches for mergers without a GRB trigger (see below). However, given the paucity of observations to date we have still to distinguish between various possible suggestions for the emission processes at play, including the role of X-ray power, the true nuclear yields and the balance between material ejected into the accretion disc or into tidal tails around the merger.

\subsection{Gravitational Wave Detection}

The mergers of compact objects were long expected to be the first observed gravitational wave signatures, and indeed NS-NS and NS-BH were long considered prime suspects for this. The recent discovery of a binary black hole merger with a particularly high total mass $\left(>60 \mathrm{M}_{\odot}\right.$ ) is therefore rather surprising at first sight (Abbott et al. 2016b), although it should be noted that the significantly higher masses here than normally considered result in a far larger horizon for massive BH-BH than for NS-NS systems (the measured strain scales 
approximately as $M_{1} M_{2}$ ), and so a significant astrophysical population of $\mathrm{BH}-\mathrm{BH}$ binaries may make them preferentially detected (Abbott et al. 2016a,c). However, at the strain sensitivities now reached by the next generation gravitational wave detectors, and given the inferred rates of NS-NS and NS-BH mergers from both population synthesis and observed populations (see below and Abadie et al. (2010)) it is expected that mergers containing neutron stars could be found shortly.

The simultaneous detection of GW and GRB signals offers significant advantages. The GRB signal provides a precise time and location for a GW search. This in turn dramatically reduces the number of trials that must be run on the gravitational wave interferometer data stream, and means that the effective sensitivity increases substantially, perhaps by a factor $\sim 2$ or more (e.g. Dietz et al. 2013). However, these advantages also extend to the study of short GRB progenitors. For example, the chirp mass determined by the GW detection would immediately identify a system as either a NS-NS or NS-BH merger. Indeed, the combination of GRB, KNe, and GW detection provides a series of different routes to probing the compact binary population over very different distance scales (see Fig. 6). In particular, SGRBs can be detected out to $z \sim 1$ or beyond (e.g. Graham et al. 2009; Thöne et al. 2011b), but likely only illuminate a small fraction of the sky due to their relativistic jets. This makes the probability of joint GRB-GW triggers relatively small, as the fraction of mergers in a volume limited sample where the GRB jet is aligned with the observer is low. Beaming is highly uncertain in short-GRBs, but beaming factors $>10-100$ seem likely (e.g. Chen and Holz 2013). This is somewhat offset since the relativistic jets are visible to observers face on to the merging binary, a geometry which also maximises the detectability of the gravitational waves (see Fig. 6 and Nissanke et al. 2011). The most promising counterparts are therefore systems that may emit isotropically. Kilonova are visible to sensitive IR searches over distances of several hundred Mpc, well matched to the sensitivities of current gravitational wave detectors. Similarly, isotropic X-rays with modest luminosity $\left(L_{X} \sim 10^{43} \mathrm{erg} \mathrm{s}^{-1}\right.$ ) should also be visible to $S$ wift or other X-ray telescopes out to distances $>100 \mathrm{Mpc}$. It is likely that both gravitational wave detections, and observations from the $\gamma$-ray to IR, and possibly beyond (for example magnetars should provide long-lived radio emission, e.g. Gompertz et al. 2015; Fong et al. 2016) will be necessary to fully constrain compact object mergers, their rate, and their role in r-process production.

\section{Central Engines: Linking the Most Luminous Explosions}

While progress towards the nature of the progenitor stars of long GRBs has been impressive there has been increasing focus on the nature of the compact object formed in the core of the collapsing star. These objects are the engines which drive the explosions and create the ultra-relativistic jets which ultimately pierce the star. Traditional models extract the GRB energy through some form of accretion onto a nascent black hole (Woosley 1993; Fryer et al. 1999). Accretion rates of up to several solar masses per second at typical efficiencies $(\sim 10 \%)$ can create the necessary luminosities to explain both long and short GRBs (e.g. Oechslin and Janka 2006), provided that a sufficiently low density of baryons exists, which seems plausible along the rotation axis of rapidly rotating stars. Recently there has been a significant shift of focus to consider energy input from a millisecond-magnetar, a newly formed highly magnetic neutron star with $B>10^{14} \mathrm{G}$, and spin periods of a few milliseconds (Metzger et al. 2011). Note that these, newly formed magnetars are quite different from the population of magnetars identified in the Milky Way as Soft Gamma Repeaters (SGRs) 


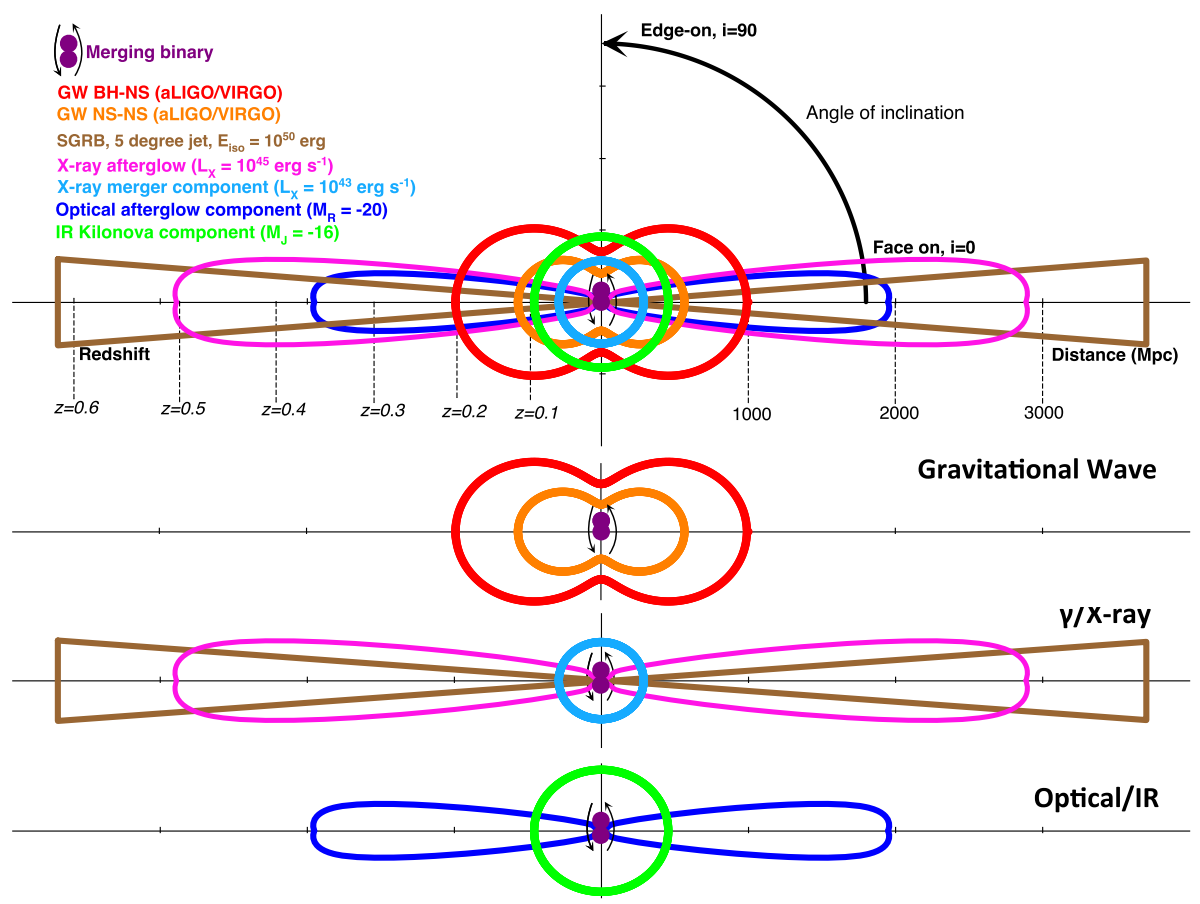

Fig. 6 The characteristic visibility horizons for the detection of different observational manifestations of compact object mergers. The top panel shows all the likely signatures overplotted for comparison, while they are broken down by detection route in the lower panels. Mergers can be detected by the Advanced generation of LIGO and VIRGO detectors out to hundreds of Mpc for both NS-NS and NS-BH mergers (Nissanke et al. 2011), although there is a strong bias towards face-on events, which can be observed to distances factors of $>2$ larger than for edge on systems. This increases the probability of observing them in coincidence with short GRBs, which are also likely to be visible only to face on observers. However, such overlapping events will likely remain in a minority. Other wavelengths of emission are likely to require triggered observations after the detection of a gravitational wave or short-GRB trigger. The horizons given are based on typical depths likely to be achieved fairly short exposures, several hours-days after the trigger with the Swift BAT $\left(S_{\gamma}=1 \times 10^{-7} \mathrm{erg} \mathrm{cm}^{-2}\right.$, although the BAT in practice triggers on short GRBs based on their peak flux), Swift XRT (limiting $F_{X} \sim 1 \times 10^{-12} \mathrm{erg} \mathrm{s}^{-1} \mathrm{~cm}^{-2}$ in $\sim 1000 \mathrm{~s}$ ), ground based robotic optical telescopes $(\mathrm{R}=21)$ and the VISTA survey telescope in the $\mathrm{IR}(\mathrm{J}(\mathrm{AB}) \sim 22)$. These horizons can obviously be scaled to different depths or time after trigger for a given assumption of temporal behaviour. In particular, observations of short-GRBs require much smaller areal coverage and so probe much deeper and farther. For example, $H S T$ can see kilonova signatures out to $z \sim 0.4$. Furthermore, uncertainty as to the detailed properties of the emission means that any such horizons should be viewed as indicative rather than precise. In particular, we properties of off-axis GRB emission which enables bursts to be viewed at larger angles than a direct GRB remains to be directly tested, and the properties of isotropic optical/IR (Barnes and Kasen 2013) or X-ray kilonova/macronova (Kisaka et al. 2015; Sun et al. 2016) that might be powered either directly by radioactivity, or by the action of a central engine such as a magnetar, remain uncertain. For SGRBs beamed signals have been the dominant source of information to date, however, it seems likely that isotropic signatures will be more promising in the majority of gravitational wave transients

and Anomalous X-ray Pulsars. The Galactic systems have typically lower fields, and importantly much slower rotation rates (several seconds) than those powering GRBs (see e.g., Olausen and Kaspi 2014). Indeed, even if the spin-down evolution of the Galactic magnetars is reversed, it seems unlikely that their early properties were consistent with those postulated as GRB central engines (Rea et al. 2015). The strong magnetic fields result in rapid extrac- 
tion of energy from the dipole field, and the total energy extracted can approach $10^{52} \mathrm{erg}$, comparable to the typical isotropic energy releases of GRBs, and significantly above the beaming corrected values. Indeed an apparent upper limit on the kinetic energy releases of GRB-supernovae of around $10^{52} \mathrm{erg}$ provides additional support for such a model (Mazzali et al. 2014). These millisecond-magnetars would eventually spin down to much longer periods, at which point they would either become dormant or, depending on their mass may collapse to black holes at the point at which they cease to be centrifugally supported.

In any case, it is clear that in the majority of GRBs this central engine inputs a large fraction of a solar rest mass on a timescale of tens to hundreds of seconds, perhaps extending to several thousand seconds in some cases. Indeed, evidence for prolonged energy injection existed before the launch of Swift (Vaughan et al. 2004; Watson et al. 2006), but was only clearly identified following the direct detection of X-ray flares in the afterglows bursts early in the Swift era (Burrows et al. 2005; Nousek et al. 2006), and was surprisingly found in both long bursts and short bursts (Berger et al. 2005). While in long bursts this late activity might naturally arise from processes involving fallback accretion, such emission was not naturally expected in the case of short GRBs should they arise from neutron star mergers, since the merger itself should be over very rapidly, with a relatively clean environment. Hence, if a common origin for the long and short GRBs was in accretion onto the nascent black hole we might expect the clean environment to provide a significant observational distinction between the two scenarios. While short GRB afterglows are markedly fainter, the presence of long-lived emission does not offer such easy solutions. This may be due to instabilities that build up in the accretion discs around both long and short GRBs and deliver similar flares (Perna et al. 2006a), or because some fraction of short GRBs arise from black hole-neutron star mergers, in which the neutron star is tidal shredded over several pericenter passages (e.g. Davies et al. 2005).

However, the millisecond-magnetar model may be particularly appealing here. Millisecond-magnetars are likely created from the collapse of some fraction of massive stars (see below) while they could also be formed via the merger of either white dwarfs or neutron stars, provided the final mass is below the maximum mass of a neutron star (Usov 1992; Levan et al. 2006). These neutron stars with extreme magnetic fields can have rapid spindown times, or may even be unstable and centrifugally supported, such that as magnetic braking slows their rotation from $\sim 1 \mathrm{~ms}$ at birth they ultimately collapse to form black holes. Importantly, millisecond-magnetars provide a route to providing energy input into the GRB afterglow on timescales of minutes to hours (or potentially even longer) after the initial burst. While the models have numerous free parameters, millisecond-magnetar models can explain many long and short GRB light curves (e.g. Rowlinson et al. 2013). However, this injection of energy is not without its problems, since it should in many cases yield a strong radio afterglow. The absence of such afterglows may be difficult to remedy with rapidly spinning magnetar models (e.g. Fong et al. 2016).

Interestingly, central engine models have become increasingly popular in explaining not only GRBs but also the most luminous SNe. In this case, the energy extracted from the millisecond-magnetar re-energises the outgoing supernova shock and creates the additional luminosity, boosting the original luminosity by a factor of 100 or more (Kasen and Bildsten 2010). The difference between GRB magnetars and those postulated in the SLSNe lies in the duration of the energy input. Millisecond-magnetars driving SNe must do much of their energy injection at late times when the supernova is large, otherwise, the energy may do work on the ejecta (increasing the ejecta velocity) but not create luminosity. In contrast, in most GRBs, the spin-down times of the millisecond-magnetars are short, such that most of their work is done more quickly. Broadly speaking GRB millisecond magnetars require high 
fields $\left(B \sim 10^{16} \mathrm{G}\right)$ and spin down on timescales of hundreds of seconds, those powering SLSNe have more modest fields $\left(B \sim 10^{14-15} \mathrm{G}\right)$ but spin down scales of days to weeks (e.g. Metzger et al. 2015). The ultra-long GRBs (Levan et al. 2014a) offer an interesting intermediate population in which millisecond-magnetars may be active for hours to days, but not longer. In this case the detection of a luminous (although not super luminous) supernova, SN2011kl associated with GRB 111209A (Greiner et al. 2015a) is of particular interest in providing evidence of a direct link between the progenitors of GRBs and those of SLSNe.

While central engine models are now increasingly used to explain a variety of exotic explosions, it is important to note that the engines themselves are not the same. Indeed, both black hole accretion and the magnetic extraction of rotational energy are considered as routes. The stars that create either black holes or rapidly spinning neutron stars may, in fact, be quite different immediately prior to the collapse, and so distinguishing between the different models is important, not only in understanding the explosions but in characterising their progenitors. Such a task is not trivial since the central engine itself is hidden at the core of the explosion. While the difference between asymmetric supernovae and ringing down magnetars is potentially distinguishable via gravitational wave observations (see e.g., Fryer et al. 2001; Davies et al. 2002; Rowlinson et al. 2013), such work likely beyond the capability of the current generation of detectors, that can only see such signatures for very local (e.g. local group) supernovae. However, the rate of energy deposition and the total energy budget are different between the two models. For example, in the millisecond-magnetar case rotational energy is released following $\dot{E}_{\text {rot }}=I \Omega \dot{\Omega}$, where $E_{r o t}$ is the rotational energy, $I$ the moment of inertia and $\Omega$ the spin frequency. In this case the total energy budget is $E_{r o t}=\frac{1}{2} I \Omega^{2}$ (Lorimer and Kramer 2004). For a $1.4 \mathrm{M}_{\odot}$ neutron star, spinning at a period of a millisecond (approximately the maximum spin rate for most neutron star equations of state) the total energy is of order $E_{\text {rot }} \sim 10^{52} \mathrm{erg}$. Alternatively, in the black hole case, the late time accretion is likely to follow the fall-back rate of $t^{-5 / 3}$, and the total energy is governed simply by the total mass accreted $E_{a c c}=\eta m_{a c c} c^{2}$ or $\dot{E}_{a c c}=\eta \dot{m}_{a c c} c^{2}$. For an efficiency of $10 \%$ and a massive star with $\sim 10 \mathrm{M}_{\odot}$ of material in-falling (either directly, or via fallback) the total energy is a much larger $E_{a c c} \sim 2 \times 10^{54} \mathrm{erg}$. Although the emission geometry can make it difficult to measure the true total energy of a given explosion, it is apparent that the most energetic GRBs and supernovae appear to exceed the limit for neutron star energy (e.g. Metzger et al. 2015), potentially posing a challenge to such models. However, there are some possibilities, such as very massive neutron stars (Metzger et al. 2015), that can provide a modest boost (factors of a few) to the total rotational energy, and so do enable such models to remain plausible, even at the high energy end of the distribution.

\section{Massive Star Progenitors}

\subsection{The Role of Rotation}

Observations make it clear that massive stars are now required in at least the vast majority, and probably all, long-duration GRBs. These massive stars must have somehow lost their hydrogen and helium envelope, create significant quantities of nickel, and have locations in galaxies consistent with the youngest and most massive stars. However, it is likely that not all stars with these conditions will launch a GRB since it would require very narrow beaming angles in GRBs for the GRB rate to match the massive star rate e.g. 1 degree for progenitors with $M_{\text {ZAMS }}>40 \mathrm{M}_{\odot}$ (Podsiadlowski et al. 2004). Indeed, the creation of a GRB is likely to require the specific conditions that can give rise to a central engine to power the burst. In the 
black hole engine model a centrifugally supported disc is formed outside a newly created black hole, the GRB jet can then be launched either through electrodynamic processes, or via neutrino-antineutrino annihilation off the disc (note that the $T^{2}$ dependence of the weak interaction cross section means that at the extreme temperatures of these discs the cross section for neutrino interactions is significant). Alternatively, in millisecond-magnetar models, the magnetar must be created, and have sufficient field and spin to energise the explosion. In both of these scenarios a crucial factor in the creation of the GRB itself arises from rotation.

In particular, the critical rotation is that of the core immediately prior to core collapse. In order to create a centrifugally supported accretion disc at the innermost stable orbit of a black hole a minimum specific angular momentum $(j)$ is required (i.e. $j$ is the angular momentum $(L)$ per unit mass $(M)$, so $j=L / M)$;

$$
j>\frac{\sqrt{6} G M}{c} .
$$

For typical core parameters this corresponds to $j>10^{16} \mathrm{~cm}^{2} \mathrm{~s}^{-1}$. A newly formed millisecond-magnetar (radius $\sim 10-20 \mathrm{~km}$, and spin period $1 \mathrm{~ms}$ ) has a rather similar specific angular momentum, and so the rotation properties of the core prior to core collapse are likely the same for the millisecond-magnetar or black hole scenarios.

In principle, such angular momentum should be easy to attain. As the core grows (and increases in density) during the main sequence its rotation should increase, potentially even to the point of breakup where the centrifugal force at the equator is equal to the gravitational force. However, in practice, angular momentum is effectively transported outwards in stars, both from the core to the envelope and subsequently from the envelope into the interstellar medium. The latter stage of this process is well understood, since mass loss from the star (at its surface) carries away angular momentum, resulting in a star with lower specific angular momentum. This process is strongly metallicity dependent, with the mass loss rate of iron group elements scaling broadly as metallicity, $Z^{0.7-0.8}$ (Vink et al. 2001; Vink and de Koter 2005). Angular momentum transport within the star is less well understood, although it seems likely that magnetic torques would be created by differential rotation of the core relative to the outer layers, and that these would ultimately create a rotation of the core that was tied to that of the envelope (Spruit 2002). Indeed, while such a model is far from universally accepted, its use does provide a reasonable match to the spins observed in neutron stars (Heger et al. 2005; Suijs et al. 2008). Since this mechanism couples the rotation of the core to that of the envelope, it therefore follows that the core is effectively braked by radiatively driven mass loss (Langer 1998), and so even at modest metallicity (e.g. somewhat less than solar) most massive stars will fall short of the critical specific angular momentum by an order of magnitude or more. Indeed, the apparent lack of angular momentum in most stars creates a signicant problem in understanding GRB creation. GRB-SNe are exclusively hydrogen poor events requiring no hydrogen envelope. However, standard pictures to create stars of this type remove the envelope via winds or binary interactions, through which significant angular momentum is also lost. At first sight, then the requirements of hydrogen deficiency and rapid rotation cannot both easily be met. However, there are solutions that may provide the necessary conditions for GRB creation.

\subsection{Single Star Scenarios}

We can consider solutions that involve both single and binary stars. In this sense we take single stars to mean stars that have either lived their entire lives (from zero age main sequence onwards) as single stars, or those in wide binaries in which there is no significant 
interaction of the two components. "Normal" massive single stars evolve to have a strong central concentration, in which material in the core is used as the fuel for fusion of progressively heavier elements. On the main sequence, this core gradually builds from hydrogen to helium, and the outer layers of the star remain (chemically) as they were at the beginning of the main sequence. However, in rapidly rotating stars the mixing timescale can be shorter than the nuclear timescale, such that material synthesised in the core of the star is broadly mixed throughout the star, creating a star which undergoes so-called chemically homogeneous evolution (e.g. Yoon and Langer 2005; Woosley and Heger 2006; Yoon et al. 2012). This is possible because rotation creates hydrodynamic instabilities at the boundary between the convective core (where hydrogen fusion occurs) and the radiative envelope of the star. Of particular importance is the Eddington-Sweet circulation (Eddington 1926; Sweet 1950) which is driven by a thermal imbalance in rotating stars and may result in a short timescale of chemical mixing with a sufficiently high rotation rate. Because these stars evolve homogeneously they do not create the standard core-envelope structure, do not undergo giant branch phases, and do not experience the core-envelope breaking that dramatically slows rotation in the cores of slowly rotating massive stars. However, this phase must be initiated early in the life of the star before a significant chemical gradient at the boundary between the hydrogen-burning convective core and the radiative envelope is built up, as otherwise, rotationally-induced mixing becomes too inefficient to make the star undergo the chemically homogeneous evolution. This process of chemically homogeneous evolution further removes the hydrogen from the star (since it is burned) while not resulting in such significant mass loss, enabling relatively massive cores to be built from more modest initial masses (Yoon and Langer 2005).

However, chemically homogeneous evolution is not common. There is little evidence for it in massive stars in the Milky Way, although observations of massive stars in the Magellanic Clouds do show some that match the expectations of chemically homogeneous evolution (Martins et al. 2009). This suggests that there may well be some metallicity dependence on stars which undergo such an evolutionary pathway. Indeed, angular momentum loss due to stellar winds is important even early in the lives of stars, so that stars have a certain threshold rotational velocity which is a function of both their stellar mass and chemical composition. The overall impact of this is that single stars can only evolve homogeneously, and hence create GRBs if they are of low metallicity. Indeed, the predicted rates of GRBs from chemically homogeneous stars drop rapidly at metallicity around $0.2 \mathrm{Z}_{\odot}$ (see Fig. 7). Interestingly, this is very similar to the critical metallicity of $0.3 \mathrm{Z}_{\odot}$ inferred from detailed observations of some host galaxies by Graham and Fruchter (2015a), but somewhat lower than that inferred from larger samples (typically at higher redshift) either via spectroscopy (Krühler et al. 2015) or photometric proxies (Perley et al. 2015). However, at these low metallicities, the contribution of these effectively single stars could be very important.

\subsection{Binary Star Scenarios}

Binary stars also provide a natural route of retaining or gaining angular momentum. In particular, stars may be spun up either via the accretion of material with high specific angular momentum, via tidal locking in tight binaries, or possibly even by direct mergers. It is pertinent to understand binary population properties in general, since these populations, in turn,

\footnotetext{
${ }^{7}$ Indeed, it has been suggested that these wide binaries are actually the best test-beds for single stars, since when observing a single star it is extremely difficult to tell if it has formerly been in a binary (Sana et al. 2012).
} 


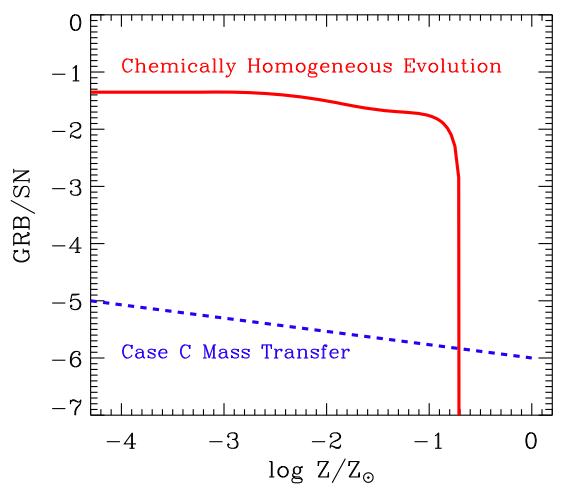

Fig. 7 The expected relative rates for the creation of rapidly rotating massive stellar cores through late binary mass transfer after the main sequence (so called case $\mathrm{C}$, blue line Podsiadlowski et al. 2010), and through chemically homogeneous evolution (red line Yoon et al. 2006). Both are plotted as a function of the metallicity of the star. It is clear that at low metallicities the effectively single star rate is much larger than the Case $\mathrm{C}$ mass transfer rate, and so will dominate over it as a route to GRB creation. In practice massive rapidly rotating stellar cores can be created through routes other than case $\mathrm{C}$ mass transfer (mergers etc.), but none-the-less it appears that a significant contribution from homogeneously evolving stars is likely at low metallicities. Any high metallicity events would necessarily arise through binary routes

provide direct constraints on the rate of much rarer events, such as those giving rise to both long and short GRBs. It is now well-established, at least in the general field of our Milky Way galaxy and in low-density star-forming regions, that of the most massive stars at least $60 \%-80 \%$ occur in main sequence-main sequence binary systems (e.g Mason et al. 1998; Kouwenhoven et al. 2005; Raghavan et al. 2010; Sana et al. 2010, 2011, 2012; Kobulnicky et al. 2014).

The highest binary fractions involving massive, and hence young, stars are found in populous compact star clusters (e.g. Elson et al. 1998; Hu et al. 2010, and references therein), interestingly, the places where the youngest most massive stars are likely to reside. This is intuitively exemplified by the much higher fraction of low- and high-mass X-ray binaries found in both globular clusters and actively star-forming regions (e.g. Coleiro and Chaty 2013) than among the general field star population. Indeed, interactions in the dense cores of these clusters tend to leave the most massive stars in binaries through exchange interactions. These simultaneously harden tight binaries, at the expense of widening, and potentially unbinding the wider binaries. This so-called hard/soft divide means that tight (hard) binaries get tighter, with their individual components more likely to interact and wider (soft) binaries become progressively wider, and less likely to interact (e.g. Heggie 1975).

Given the apparent preference that GRBs have for low(er) metallicity environments, studies in the Magellanic Clouds may be particularly insightful. For example, studies of the population in the 15-30 Myr-old compact Large Magellanic Cloud (LMC) star clusters NGC 1818 (Hu et al. 2010; de Grijs et al. 2013; Li et al. 2013), show that for binaries with an F-star primary and mass ratios, $q \equiv m_{2} / m_{1}>0.4$ (where $m_{1}$ and $m_{2}$ are the masses of the primary and secondary components, respectively), the cluster's binary fraction is $\sim 0.35$. This suggests a total binary fraction for F stars of 0.55 to unity, depending on assumptions about the form of the mass-ratio distribution at low $q$. A similarly high binary fraction (covering the clusters' full observable mass ranges) is obtained for the equivalently young, compact cluster NGC 1805 (Li et al. 2014), as well as for the intermediate-age ( $\sim$ a few $\times 10^{8} \mathrm{yr}$-old), massive LMC clusters NGC 1831 and NGC 1868 (Li et al. 2014). 
However, these binary fractions represent the entire population of binary systems. In a surprising development de Grijs et al. (2013), noticed that the binary fraction in the core region of NGC 1818 was significantly lower than that in the cluster's outer regions and even in the surrounding field. It seems likely that this is the effect of the dynamical disruption of soft binaries on short timescales in the dense inner core of the cluster where interaction times are low. Indeed, subsequent $\mathrm{N}$-body simulations confirm this scenario (Geller et al. 2013).

For a range of initial conditions, from smooth virialized density distributions to highly substructured and collapsing configurations it is possible to explain the structures of the cluster, although with a slight preference for structured initial conditions. These models produce the observed radial trend in binary frequency through disruption of soft binaries (with semi-major axes, $a>3000 \mathrm{AU}$ ), on approximately a crossing time, preferentially in the cluster core. Mass segregation subsequently causes the remaining binaries to sink towards the core (Geller et al. 2015). Thus, both a radial binary fraction distribution that falls towards the core (as observed for NGC 1818) and one that rises towards the core (as for dynamically older star clusters) can arise naturally from the same evolutionary sequence owing to binary disruption and mass segregation in rich star clusters. Indeed, the radial distribution of the binary fraction in another very young LMC cluster NGC 1805, showed an enhanced binary fraction in its core. This cluster is dynamically much older than NGC 1818, however, so that the effects of dynamical mass segregation had already become dominant (Geller et al. 2015). Such complex evolution within clusters means that studies of locations that attempt to derive the binarity of GRB progenitors (e.g. due to kicks to the binaries during a previous supernova explosion) are challenging since their initial locations might be widely varied.

Once binaries are formed, their evolution is dictated both by the evolution of the individual components, but crucially, also by the interaction between the two stars, which can have a marked impact on their evolutionary pathways. The introduction of binary channels greatly increases the diversity of stellar evolution pathways and their associated remnants. Indeed, it is visible on galaxy-wide scales where, for example, the rejuvenation of older stars via binary interactions can make them look much younger than they (or the bulk of the stellar population) really are (Eldridge and Stanway 2009; Stanway et al. 2016).

Because of the increased diversity of stellar products that can be created through binary evolution (e.g. unusual composition, rotation, mass etc), binary channels are popular routes to create exotic (and rare) stellar transients. Through binary evolution it is straightforward to remove the stellar envelope (and provide a stripped envelope core collapse supernova), while tidal locking in tight binaries can in principle provide the necessary angular momentum in the core to enable disc or millisecond-magnetar formation. However, the impacts of binary evolution go beyond the straightforward changes on the outer layers of the star due to the impact of centrifugal force and rotationally-induced chemical mixing. Hence, detailed studies require the construction of complex models that can simultaneously model angular momentum transport within individual stars and the binary components, as well as tracking mass loss and nuclear burning. Such calculations have been attempted by several teams (e.g. Yoon et al. 2010).

As with single stars, the crucial parameter to track through binary routes is the specific angular momentum of the stellar core immediately prior to core collapse. Stars can be spun up via mass transfer from a companion star, but are spun down if they themselves lose mass. This creates similar problems to those seen in massive single stars, in which it is very difficult to both lose the hydrogen envelope and retain both sufficient mass and rotation to obtain the necessary conditions for GRB creation. Several authors have considered late time 
mass transfer during a late stage of core helium burning or after core helium exhaustion (so-called Case $\mathrm{C}$ mass transfer) as a possible route to obtaining GRB-like conditions. This late time mass transfer can enable the formation of a massive stellar core, even at high metallicity (Brown and Lee 2004). Alternatively, depending on the properties of the binary this mass transfer may result in the formation of a common envelope, which will unbind the envelope of the star at the cost of the orbital energy (and hence separation). The resulting binary (after removal of the envelope) is a tight binary containing a carbon-oxygen core. Tidal locking within this binary may match the rotation period of the core to the period of the binary (typically a few hours) in which case the requirements on specific angular momentum can be met (e.g. Lee et al. 2002). Indeed, tidal locking through a variety of routes including neutron stars, post main sequence stars and low mass main sequence stars has been considered as a route of creating GRBs (Izzard et al. 2004; van den Heuvel and Yoon 2007), although detailed calculations disfavour this scenario (Detmers et al. 2008). Alternatively, evolution in binaries with relatively extreme mass ratios may also create the necessary conditions. Here mass transfer occurs from a massive star evolving away from the main sequence, and onto a low mass star on the main sequence. In this scenario, a further mass transfer from the low mass companion can occur on the CO core of the primary star. This, in turn, leads to both the spin up of the core, and the explosive ejection of the common envelope (Podsiadlowski et al. 2010).

Finally, a route to obtaining the necessary angular momentum may be the direct merger of two stars. Such mergers do happen, for example, common envelope mergers have been used to explain unusual stellar outbursts of lower mass stars, such as V838 Mon, V1309 Sco or R136a1 (e.g. Tylenda and Soker 2006; Tylenda et al. 2011; Banerjee et al. 2012). More massive mergers may create the elusive Thorne-Zytkov objects. One of these has recently been claimed in the SMC (Levesque et al. 2014), although this is controversial, in particular, it seems unclear if it actually resides in the SMC (Worley et al. 2016), or is a foreground source (Maccarone and de Mink 2016). Indeed, at higher masses the merger of either two Helium cores, or perhaps a black hole (or neutron star) with a Helium core has been proposed as a GRB mechanism, with the latter suggested as a possible origin for the Christmas-day burst, GRB 101225A (e.g. Thöne et al. 2011a). In these mergers the orbital angular momentum eventually is combined within the single merged object. In the case of He-star-He-star mergers this dramatically increases the total mass, and there is little time between the merger and the supernova. For black hole-He star mergers, the black hole accretion can essentially create the GRB immediately.

It is clear that there are a wide variety of binary channels that can give rise to the necessary conditions for GRB production. All of these scenarios are likely to occur in nature. However, it is less clear if the most exotic events can create GRBs at the necessary rate to explain the observed populations of GRBs. In each of these scenarios, there is a limited parameter space over which the channel will work (in terms of mass, mass ratio, and initial separation), and so a given route is likely to provide only a modest rate of GRB-like events (see Fig. 7). Hence, while many stars are in binaries, the rates of creation of rapidly rotating massive cores through any one of these routes is significantly lower than the rate obtained through chemically homogeneous evolution for any star formation occurring at substantially sub-solar metallicity.

\subsection{Predicted Long GRB Rates}

Determining the rates of GRBs, and of their various progenitor channels is fraught with difficulty. Observationally, GRB detection is a sensitive function of both the properties of 
the detector (area, energy range etc.) and of the burst (hardness, peak flux, total fluence etc.). Furthermore, GRBs exhibit a broad range of luminosities, and so an extrapolation to the total rate of GRB-like events requires a significant correction factor. Indeed, it is apparent that there is a population of local, low-luminosity GRBs whose volumetric rate exceeds those of the more distant bursts by a factor of several hundred. If these are a separate population, or the faint end of a luminosity function remains uncertain (e.g. Chapman et al. 2007; Liang et al. 2007b), although the similarities in the supernovae in both the lowest and highest luminosity examples (e.g. Galama et al. 2000; Pian et al. 2006b; Xu et al. 2013; Levan et al. 2014b) suggests that similar physical mechanisms are at play. Finally, GRBs are relativistically beamed, and illuminate only a small fraction of the sky (Frail et al. 2001; Bloom et al. 2003). The beaming fraction for a given burst can be obtained from the so-called jet break when the relativistic jet expands laterally (Frail et al. 2001), but is a challenging and still highly uncertain parameter that significantly impacts the observed rates.

Similarly, when determining the likely rates of GRB production via the various routes considered above there are major uncertainties that enter. What is the relevant metallicity distribution for stars throughout the Universe, and how does this impact those that will undergo chemically homogeneous evolution (Langer and Norman 2006)? Are there any special environments in which the apparently universal top end of the initial mass function can become more top heavy (Bastian et al. 2010)? What is the binary fraction, and the range of initial separations? How is the mass ratio of binaries distributed, are these universal, and might this have any impact on the final products of stellar evolution ( $\mathrm{Li}$ et al. 2013)? What is the efficiency of common envelope evolution (e.g. Nelemans and Tout 2005)? While these parameters can be studied from detailed simulations (or observations) (e.g. Yoon et al. 2006), or explored via rapid population synthesis (e.g. Izzard et al. 2004) there are still order of magnitude uncertainties in the true rate. Podsiadlowski et al. (2004) attempted to compare the rates of GRBs and massive stars by directly comparing the rates of GRBs (for some assumptions about beaming angles) with the rates of stars of a given mass within a typical galaxy. In Table 1 we provide an updated version of this table, including estimates of the low- and high- luminosity GRBs separately, as well as different types of SNe and massive stars. We also provide the rates of massive stars for a typical (i.e. Milky Way-like) galaxy, as well as the rates of massive stars below some threshold metallicity in the local Universe (extrapolated from Graham and Schady 2015b). We note that these scaled values assume a constant factor of $10^{7}$ between the volume averaged rate and the galactic rate, and so does not take into account chemical differences between galaxies of different masses.

The rate of classical, high luminosity long GRBs, for a beaming angle of a few degrees is remarkably similar to the rate of low metallicity $>40 \mathrm{M}_{\odot}$ stars observed in the local Universe. Since both low metallicity (Graham and Fruchter 2015a; Perley et al. 2015) and high mass (Larsson et al. 2007; Raskin et al. 2008) have been observationally linked to GRBs this may suggest that a reasonable fraction of initially very massive stars create long GRBs. Although such interpretation is challenging because many high-luminosity long GRBs are at much higher redshift, where star formation rate densities are higher. None-the-less, this suggests that the rate of production of long GRBs is not much lower than the rate of production of massive $\left(>40 \mathrm{M}_{\odot}\right)$, low metallicity $\left(<0.25 \mathrm{Z}_{\odot}\right)$ stars. In turn, this implies that they are probably not created only from stars which undergo very rare and unusual interactions. For example, for a typical 5-degree beaming angle, the LGRB rate is only a factor of 3 lower than the low metallicity massive star rate. While alternative (wider) beaming angles could lower the rate of GRBs by an order of magnitude or more, it still seems unlikely that the rarest channels, involving $<1 \%$ of stars are likely to be creating long GRBs. Indeed, if the 
Table 1 Approximate rates of various engine driven transients, and of massive stars at all metallicities, and below a set metallicity. Various attempts have been made to determine these rates, and the numbers given are rounded and approximate, rather than representing the full range of possibilities (which in some cases are quite large). A typical galaxy is assumed to have a B-band luminosity of $10^{10} \mathrm{~L} \odot$, and we assume a local B-band luminosity density of $10^{8} \mathrm{Mpc}^{-3}$ (e.g. Calura and Matteucci 2003), although clearly, this value evolves significantly over cosmic history. We note that the numbers per galaxy have been directly scaled from the volume averaged values with a fixed scaling of $10^{7}$. Since stars of a given chemical makeup are not the same "per galaxy" due to the mass-metallicity relation (Tremonti et al. 2004), the relative numbers of low metallicity stars should be viewed with caution since a given galaxy might contain stars entirely of low metallicity (if it was of low mass), or very few stars of low metallicity (if it was of high mass), i.e. if low metallicity is a requirement for GRB production then the rate per galaxy at low metallicity is boosted in low mass/metallicity galaxies, and depressed in high mass/metallicity galaxies. The massive star corrections to low metallicity (formally $12+\log (\mathrm{O} / \mathrm{H})<8.4$ following Graham and Schady $(2015 \mathrm{~b})$ ) are at $z \sim 0$ where $10 \%$ of the star formation lies below this metallicity. This increases rapidly, and the correction likely becomes less than a factor 2 by $z \sim 2$.

Object Rate $\left(\right.$ galaxy $\left.^{-1} \mathrm{yr}^{-1}\right)$ Rate $\left(\mathrm{Gpc}^{-3} \mathrm{yr}^{-1}\right)$ References

\section{Transients}

\begin{tabular}{|c|c|c|c|}
\hline LGRB & $8 \times 10^{-8}$ & 0.8 & Sun et al. (2015) \\
\hline$-1^{\circ}$ & $6.6 \times 10^{-4}$ & 6600 & \\
\hline$-5^{\circ}$ & $2.6 \times 10^{-5}$ & 260 & \\
\hline$-20^{\circ}$ & $1.6 \times 10^{-6}$ & 16 & \\
\hline LLGRB & $1.6 \times 10^{-5}$ & 160 & Sun et al. (2015) \\
\hline$-1^{\circ}$ & 0.11 & $1.1 \times 10^{6}$ & \\
\hline$-5^{\circ}$ & $4.2 \times 10^{-3}$ & 42000 & \\
\hline$-20^{\circ}$ & $2.6 \times 10^{-4}$ & 2600 & \\
\hline SGRB & $2.0 \times 10^{-7}$ & 2.0 & Sun et al. (2015) \\
\hline$-1^{\circ}$ & $1.3 \times 10^{-3}$ & 13000 & \\
\hline$-5^{\circ}$ & $5.3 \times 10^{-5}$ & 530 & \\
\hline$-20^{\circ}$ & $3.0 \times 10^{-6}$ & 30 & \\
\hline ULGRB & $1 \times 10^{-8}$ & 0.1 & Gendre et al. (2013), Prajs et al. (2016) \\
\hline$-1^{\circ}$ & $6.6 \times 10^{-5}$ & 660 & \\
\hline$-5^{\circ}$ & $2.5 \times 10^{-6}$ & 25 & \\
\hline$-20^{\circ}$ & $2.0 \times 10^{-7}$ & 2.0 & \\
\hline rTDE & $3.0 \times 10^{-9}$ & 0.03 & Sun et al. (2015) \\
\hline SLSNe* & $3.0 \times 10^{-6}$ & 30 & Quimby et al. (2013), Prajs et al. (2016) \\
\hline
\end{tabular}

Massive stars

$\begin{array}{lll}20 \mathrm{M}_{\odot} \text { all } Z & 2 \times 10^{-3} & 20000 \\ 20 \mathrm{M}_{\odot} Z<1 / 4 Z_{\odot} & 2 \times 10^{-4} & 2000 \\ 40 \mathrm{M}_{\odot} \text { all } Z & 6 \times 10^{-4} & 6000 \\ 40 \mathrm{M}_{\odot} Z<1 / 4 Z_{\odot} & 6 \times 10^{-5} & 600 \\ 80 \mathrm{M}_{\odot} \text { all } Z & 2 \times 10^{-4} & 2000 \\ 80 \mathrm{M}_{\odot} Z<1 / 4 Z_{\odot} & 2 \times 10^{-5} & 200\end{array}$

Podsiadlowski et al. (2004)

Graham and Schady (2015b)

\section{Compact binaries}

\begin{tabular}{llll} 
NS-NS & $6 \times 10^{-5}$ & 600 & Abadie et al. (2010) \\
NS-BH & $2 \times 10^{-7}$ & 2 & \\
BH-BH & $5 \times 10^{-7}$ & 5 & Abbott et al. (2016a) \\
\hline
\end{tabular}


low-luminosity GRBs are arising from a similar population, with similar progenitor masses and low metallicities then they would need to be born from a significant fraction of the very massive stars, even in the case of no beaming. Even with no beaming correction, the rate per galaxy of low-luminosity GRBs is $\sim 2 \times 10^{-5}$ galaxy $^{-1} \mathrm{yr}^{-1}$, again only a factor of a few lower than the low metallicity $40 \mathrm{M}_{\odot}$ stars. Indeed, since these low-luminosity GRBs are at low redshift there is less concern about evolution over cosmic history, and it seems likely that the low-luminosity GRBs are either lower-mass stars or nearly isotropic in emission in order to avoid low luminosity GRB rates that approach, or even exceed the massive star formation rate. It is also relevant to compare the rates to those of other transients, for example, the newly uncovered population of ULGRBs (Levan et al. 2014a) that may be linked to the SLSNe (Greiner et al. 2015a). The beaming corrections in this case are particularly uncertain, but for plausible beaming rates, comparable to those of long GRBs the inferred rate of ULGRBs is lower, of the same order of magnitude as the SLSNe rate. However, given the substantial uncertainties in both rate calculations, it is possible that this similarity is purely coincidental.

\section{Constraints from Local Stellar Populations}

\subsection{O Stars}

It is apparent that long GRBs arise primarily from a subset of massive $\left(M_{\text {ZAMS }} \geq 40 \mathrm{M}_{\odot}\right)$, moderately metal-poor stars at cosmological redshifts. Although the nearest long GRBs lie at distances of tens to hundreds of megaparsec, we can study individual massive stars within appropriate environments in the Local Group.

The overwhelming majority of stars are believed to have their origins in dense star clusters, intermediate density OB associations or low-density star forming regions. If stars are randomly drawn from a Kroupa (2001) Initial Mass Function (IMF), cluster masses over $\sim 100 \mathrm{M}_{\odot}$ are required to produce one star capable of ending its life as a core-collapse supernova. However, the most massive stars are preferentially found in the more massive clusters. In particular, stochastic sampling of the initial mass function means that the probability of a low mass cluster forming a very massive star is low (i.e. the massive star content of say $10,100 \mathrm{M}_{\odot}$ associations is less than that of a single $1000 \mathrm{M}_{\odot}$ cluster). Indeed, it has been suggested that the maximum mass of a star in a cluster $\left(M_{*, \max }\right)$ is related to the cluster total mass $\left(M_{C}\right)$, such that $M_{*, \max } \approx 0.39 M_{C}^{2 / 3}$ (Weidner et al. 2010). In this case, for a star forming region to include at least one star with an initial mass of at least $\sim 40 \mathrm{M}_{\odot}$, a total mass $10^{3} \mathrm{M}_{\odot}$ is required, with 10 such stars hosted by $10^{4} \mathrm{M}_{\odot}$ clusters.

The upper limit to star cluster mass is a sensitive function of how vigorously stars are being formed, so one would not expect long GRBs to occur in galaxies with low specific star formation rates. Therefore if progenitors of long GRBs arise from very massive stars, they will exclusively occur in galaxies with high specific star formation rates. Indeed, Kelly and Kirshner (2012) conclude that low $z$ broad-lined (BL) SNe Ic arise exclusively from hosts with the highest star-forming intensities, with Kelly et al. (2008) having earlier established that long GRBs and low $z$ SN Ic-BL originate from similar environments.

Since long GRBs strongly favour host galaxies with metallicities below 1/2 solar, the focus of our attention in the Local Group will be primarily the Large and Small Magellanic Clouds, with $1 / 2$ and $1 / 5$ solar metallicity, respectively. The most massive young starforming region in the SMC, N66/NGC 346, hosts several tens of O stars, whereas many hundreds of $\mathrm{O}$ stars are known in the Tarantula Nebula region of the LMC. 
The VLT FLAMES Tarantula Survey (VFTS Evans et al. 2011) has enabled properties of $800 \mathrm{OB}$ stars in this region to be determined, revealing that $50 \%$ of $\mathrm{O}$ stars are affected by binary evolution (Sana et al. 2013), with relatively low rotation rates, aside from a few very fast rotators presumably arising from binary interactions (Ramírez-Agudelo et al. 2015). Several star clusters are located within the Tarantula Nebula, most noticeably R136, the youngest (1.5 Myr) very high-mass cluster within the Local Group. Crowther et al. (2016) exploit HST/STIS spectroscopy to reveal that over three dozen stars more massive than $40 \mathrm{M}_{\odot}$ are located within the central parsec.

Nearly 30 very massive stars (VMS, $\geq 100 \mathrm{M}_{\odot}$ ) are located in the Tarantula Nebula, the majority within the R136 region, but others up to 100 parsecs away (Crowther et al. 2016). Some of the more remote VMS are plausible runaways from R136, but others likely were born in significantly low-density regions. In contrast, no VMS are located in SMC's N66 star-forming region, although it hosts a triple high mass system HD 5980.

In summary, large numbers of candidate long GRB progenitors exist in the LMC/SMC as far as their initial masses are concerned, yet there is no evidence that these are born with sufficiently high rotational rates for chemically homogeneous evolution. Rapid rotation $\left(v \sin i>500 \mathrm{~km} \mathrm{~s}^{-1}\right.$ ) is observed for small numbers of $\mathrm{O}$ stars in the Tarantula Nebula, presumably spun-up via binary evolution (de Mink et al. 2013). Similarly, no examples of rapid rotators are observed in N66 (Mokiem et al. 2006). Lamb et al. (2016) identify a large population of SMC field Oe stars, presumably arising from rapid rotation, although these too may result from close binary evolution. This in itself raises interesting questions, since if a significant population of sufficiently massive and low metallicity stars can be identified in the local Universe, but essentially none of these appear as viable GRB progenitors then the fraction of massive low metallicity stars creating GRBs must be small. However, this begins to create tension with the necessary observed rates, especially when lower luminosity GRBs are considered (see Sect. 5.4).

\subsection{Wolf-Rayet Stars}

The immediate progenitors of long GRBs are massive stars whose hydrogen-rich envelopes have been stripped away to reveal compact cores, i.e. classical Wolf-Rayet (WR) stars (Crowther 2007). These stars possess dense, fast outflows with atmospheric compositions characteristic of hydrogen-burning (WN-type) or helium-burning (WC-type). In common with their $\mathrm{O}$ type progenitors, the strength of WR winds scales with metallicity (Vink and de Koter 2005), so metal-poor WR stars possess, lower density, slower outflows than their Milky Way counterparts (Hainich et al. 2015b).

The observed association between long GRBs and SN Ic-BL suggests the presence of very little helium in the progenitor star, favouring WC stars. The Magellanic Clouds host approximately $150 \mathrm{WR}$ stars, of which only $15 \%$ belong to the carbon sequence or rare oxygen sequence (WO). The binary frequency of Magellanic Cloud WR stars is approximately $40 \%$, similar to that observed in the Milky Way, with a lower binary fraction amongst WC and WO stars (Bartzakos et al. 2001).

Analysis of single WC and WO stars in the LMC (Crowther et al. 2002; Tramper et al. 2015) reveals He core masses of 10-20 $\mathrm{M}_{\odot}$. The sole SMC carbon/oxygen sequence WR star is in a close binary system, although Shenar et al. (2016) argue that the binary channel does not dominate the formation of WR stars at this metallicity.

The dense outflows from WR stars prevent direct rotational velocity measurements, but searches for non-spherical geometries can be done via linear spectropolarimetry. None of the four LMC WC stars observed by Vink (2007) exhibited the characteristic line depolarization 
with respect to the continuum, ruling out rapid rotation in these cases. Martins et al. (2009) have claimed that at least one of the hydrogen-rich WN stars in the SMC is the product of chemically homogeneous evolution. However, this single star might represent the product of spin-up arising from close binary evolution since such high initial rotation rates are not currently established amongst massive $\mathrm{O}$ stars in the SMC.

Finally, studies have been undertaken investigating the locations of WR stars relative to their host light, in principle directly comparable to the locations of transient phenomena (Leloudas et al. 2010; Bibby and Crowther 2012). These broadly show the locations of stripped envelope SNe match those of the WR population (Bibby and Crowther 2012), and that WC stars are more concentrated on their host light than WN system (Leloudas et al. 2010).

In summary, there are no robust long GRB progenitors amongst the Magellanic Cloud WR population at present, although at least one SMC WR star has been claimed to be the result of chemically homogeneous evolution, and their weak winds make measurements of their rotational rates via spectropolarimetry extremely challenging.

\section{Summary and Open Questions}

We are now approaching 20 years since the discovery of the first GRB afterglows, and to date over 1000 have been discovered. Intensive observations of the bursts themselves, their afterglows, associated supernovae and host galaxies have provided firm links to their progenitors in several cases, and in particular the link between long GRBs and type Ic supernovae appears secure. In turn, the link to young massive stars, coupled with their extreme luminosity makes GRBs a powerful probe of the distant Universe from the discovery of some of the most distant galaxies, to detailed work studying the buildup of stellar mass and metals across cosmic history. However, while this utility is clear, there remain many questions in addressing the nature of the progenitors themselves, and this, in turn, has a systematic impact on the cosmological utility of GRBs (e.g. in determining the correct factor to convert a GRB rate to a star formation rate at a given redshift/metallicity). In this review, we have highlighted much of the progress that has been made through a combination of intensive observation, large-scale numerical calculation and direct observation of local analogue populations. However, there are many questions that remain open, and will be the focus of research in the coming years, in particular;

- What fraction of massive O-stars are really required to create GRBs? What does this mean about routes to their progenitors?

- What is the true metallicity dependence for GRB production?

- How important is binary evolution in the creation of GRBs?

- Are any of the locally observed massive, rapidly rotating stars actually likely to create a GRB? Have any in the past that we can identify?

- What is the diversity of supernovae seen in long GRBs? Can they be standard candles? Is the presence of a luminous $\mathrm{SN}$ in one case a common or extremely rare occurrence?

- What is the range of kilonova/macronova properties seen in short GRBs? Can these create an important contribution to r-process enrichment, and explain the levels seen in the seafloor on Earth?

- What is the role of NS-NS and NS-BH mergers in short GRB production? Can any BH$\mathrm{BH}$ mergers make GRBs? What will simultaneous gravitational wave signatures tell us? 
The answers to these questions are likely to come both from the application of the traditional techniques described above, with improved telescope aperture and response (e.g. JWST, E-ELT, GMRT etc.), or the every increasing computational power, and through new routes, such as direct multi-messenger observations (gravitational waves, neutrinos) that are now producing astrophysical detections. The answers to these questions are challenging, but it is likely that many will be uncovered with a degree of certainty in the coming decade.

Acknowledgements We thank the organisers and ISSI Beijing for a productive and well organised meeting in April 2015, and the referee for a very constructive and valuable report that has improved this manuscript. AJL acknowledges support from the Science and Technology Facilities Council under programme ST/L000733/1 and from the Philip Leverhulme Trust via a Leverhulme Prize. He is grateful for productive conversations with Nial Tanvir. RdG acknowledges financial support from the National Natural Science Foundation of China (grants 11373010, 11633005 and U1631102). He also acknowledges helpful insights into the evolution of binary systems provided by the participants of the 2014 conference on 'Binary systems, their evolution and environments' (particularly Philipp Podsiadlowski, Chris Belczyński and Dany Vanbeveren). SCY was supported by the Basic Science Research (2013R1A1A2061842) program through the National Research Foundation of Korea (NRF).

Open Access This article is distributed under the terms of the Creative Commons Attribution 4.0 International License (http://creativecommons.org/licenses/by/4.0/), which permits unrestricted use, distribution, and reproduction in any medium, provided you give appropriate credit to the original author(s) and the source, provide a link to the Creative Commons license, and indicate if changes were made.

\section{References}

J. Abadie, B.P. Abbott, R. Abbott, M. Abernathy, T. Accadia, F. Acernese, C. Adams, R. Adhikari, P. Ajith, B. Allen et al., Topical review: predictions for the rates of compact binary coalescences observable by ground-based gravitational-wave detectors. Class. Quantum Gravity 27(17), 173001 (2010). doi:10.1088/0264-9381/27/17/173001

B.P. Abbott, R. Abbott, T.D. Abbott, M.R. Abernathy, F. Acernese, K. Ackley, C. Adams, T. Adams, P. Addesso, R.X. Adhikari et al., Astrophysical implications of the binary black hole merger GW150914. Astrophys. J. Lett. 818, 22 (2016a). doi:10.3847/2041-8205/818/2/L22

B.P. Abbott, R. Abbott, T.D. Abbott, M.R. Abernathy, F. Acernese, K. Ackley, C. Adams, T. Adams, P. Addesso, R.X. Adhikari et al., Observation of gravitational waves from a binary black hole merger. Phys. Rev. Lett. 116(6), 061102 (2016b). doi:10.1103/PhysRevLett.116.061102

B.P. Abbott, R. Abbott, T.D. Abbott, M.R. Abernathy, F. Acernese, K. Ackley, C. Adams, T. Adams, P. Addesso, R.X. Adhikari et al., The Rate of Binary Black Hole Mergers Inferred from Advanced LIGO Observations Surrounding GW150914. ArXiv e-prints (2016c)

L. Amati, F. Frontera, C. Guidorzi, Extremely energetic Fermi gamma-ray bursts obey spectral energy correlations. Astron. Astrophys. 508, 173-180 (2009). doi:10.1051/0004-6361/200912788

J.P. Anderson, S.M. Habergham, P.A. James, M. Hamuy, Progenitor mass constraints for core-collapse supernovae from correlations with host galaxy star formation. Mon. Not. R. Astron. Soc. 424, 1372-1391 (2012). doi:10.1111/j.1365-2966.2012.21324.x

C.R. Angus, A.J. Levan, D.A. Perley, N.R. Tanvir, J.D. Lyman, E.R. Stanway, A.S. Fruchter, A Hubble Space Telescope survey of the host galaxies of superluminous supernovae. Mon. Not. R. Astron. Soc. 458, 84-104 (2016). doi:10.1093/mnras/stw063

Z. Arzoumanian, D.F. Chernoff, J.M. Cordes, The velocity distribution of isolated radio pulsars. Astrophys. J. 568, 289-301 (2002). doi:10.1086/338805

S. Banerjee, P. Kroupa, S. Oh, The emergence of super-canonical stars in R136-type starburst clusters. Mon. Not. R. Astron. Soc. 426, 1416-1426 (2012). doi:10.1111/j.1365-2966.2012.21672.x

J. Barnes, D. Kasen, Effect of a high opacity on the light curves of radioactively powered transients from compact object mergers. Astrophys. J. 775, 18 (2013). doi:10.1088/0004-637X/775/1/18

P. Bartzakos, A.F.J. Moffat, V.S. Niemela, Magellanic cloud WC/WO Wolf-Rayet stars-I. Binary frequency and Roche lobe overflow formation. Mon. Not. R. Astron. Soc. 324, 18-32 (2001). doi:10.1046/ j.1365-8711.2001.04126.x

N. Bastian, K.R. Covey, M.R. Meyer, A universal stellar initial mass function? A critical look at variations. Annu. Rev. Astron. Astrophys. 48, 339-389 (2010). doi:10.1146/annurev-astro-082708-101642 
E. Berger, The host galaxies of short-duration gamma-ray bursts: luminosities, metallicities, and starformation rates. Astrophys. J. 690, 231-237 (2009). doi:10.1088/0004-637X/690/1/231

E. Berger, A short gamma-ray burst "No-host" problem? Investigating large progenitor offsets for short GRBs with optical afterglows. Astrophys. J. 722, 1946-1961 (2010). doi:10.1088/0004-637X/722/2/1946

E. Berger, W. Fong, R. Chornock, An r-process kilonova associated with the short-hard GRB 130603B. Astrophys. J. Lett. 774, 23 (2013). doi:10.1088/2041-8205/774/2/L23

E. Berger, P.A. Price, S.B. Cenko, A. Gal-Yam, A.M. Soderberg, M. Kasliwal, D.C. Leonard, P.B. Cameron, D.A. Frail, S.R. Kulkarni, D.C. Murphy, W. Krzeminski, T. Piran, B.L. Lee, K.C. Roth, D.-S. Moon, D.B. Fox, F.A. Harrison, S.E. Persson, B.P. Schmidt, B.E. Penprase, J. Rich, B.A. Peterson, L.L. Cowie, The afterglow and elliptical host galaxy of the short $\gamma$-ray burst GRB 050724. Nature 438, 988-990 (2005). doi:10.1038/nature04238

E. Berger, D.B. Fox, P.A. Price, E. Nakar, A. Gal-Yam, D.E. Holz, B.P. Schmidt, A. Cucchiara, S.B. Cenko, S.R. Kulkarni, A.M. Soderberg, D.A. Frail, B.E. Penprase, A. Rau, E. Ofek, S.J.B. Burnell, P.B. Cameron, L.L. Cowie, M.A. Dopita, I. Hook, B.A. Peterson, P. Podsiadlowski, K.C. Roth, R.E. Rutledge, S.S. Sheppard, A. Songaila, A new population of high-redshift short-duration gamma-ray bursts. Astrophys. J. 664, 1000-1010 (2007). doi:10.1086/518762

D. Bersier, A.S. Fruchter, L.-G. Strolger, J. Gorosabel, A. Levan, I. Burud, J.E. Rhoads, A.C. Becker, A. Cassan, R. Chornock, S. Covino, R.S. de Jong, D. Dominis, A.V. Filippenko, J. Hjorth, J. Holmberg, D. Malesani, B. Mobasher, K.A.G. Olsen, M. Stefanon, J.M. Castro Cerón, J.P.U. Fynbo, S.T. Holland, C. Kouveliotou, H. Pedersen, N.R. Tanvir, S.E. Woosley, Evidence for a supernova associated with the X-ray flash 020903. Astrophys. J. 643, 284-291 (2006). doi:10.1086/502640

J.L. Bibby, P.A. Crowther, The Wolf-Rayet population of the nearby barred spiral galaxy NGC 5068 uncovered by the very large telescope and gemini. Mon. Not. R. Astron. Soc. 420, 3091-3107 (2012). doi:10.1111/j.1365-2966.2011.20220.x

J.S. Bloom, D.A. Frail, S.R. Kulkarni, Gamma-ray burst energetics and the gamma-ray burst Hubble diagram: promises and limitations. Astrophys. J. 594, 674-683 (2003). doi:10.1086/377125

J.S. Bloom, S.R. Kulkarni, S.G. Djorgovski, The observed offset distribution of gamma-ray bursts from their host galaxies: a robust clue to the nature of the progenitors. Astron. J. 123, 1111-1148 (2002). doi: $10.1086 / 338893$

J.S. Bloom, S.R. Kulkarni, S.G. Djorgovski, A.C. Eichelberger, P. Côté, J.P. Blakeslee, S.C. Odewahn, F.A. Harrison, D.A. Frail, A.V. Filippenko, D.C. Leonard, A.G. Riess, H. Spinrad, D. Stern, A. Bunker, A. Dey, B. Grossan, S. Perlmutter, R.A. Knop, I.M. Hook, M. Feroci, The unusual afterglow of the $\gamma$-ray burst of 26 March 1998 as evidence for a supernova connection. Nature 401, 453-456 (1999). doi:10. $1038 / 46744$

J.S. Bloom, J.X. Prochaska, D. Pooley, C.H. Blake, R.J. Foley, S. Jha, E. Ramirez-Ruiz, J. Granot, A.V. Filippenko, S. Sigurdsson, A.J. Barth, H.-W. Chen, M.C. Cooper, E.E. Falco, R.R. Gal, B.F. Gerke, M.D. Gladders, J.E. Greene, J. Hennanwi, L.C. Ho, K. Hurley, B.P. Koester, W. Li, L. Lubin, J. Newman, D.A. Perley, G.K. Squires, W.M. Wood-Vasey, Closing in on a short-hard burst progenitor: constraints from early-time optical imaging and spectroscopy of a possible host galaxy of GRB 050509b. Astrophys. J. 638, 354-368 (2006). doi:10.1086/498107

O. Bromberg, E. Nakar, T. Piran, R. Sari, Short versus long and collapsars versus non-collapsars: a quantitative classification of gamma-ray bursts. Astrophys. J. 764, 179 (2013a). doi:10.1088/0004-637X/ $764 / 2 / 179$

O. Bromberg, E. Nakar, T. Piran, R. Sari, Short versus long and collapsars versus non-collapsars: a quantitative classification of gamma-ray bursts. Astrophys. J. 764, 179 (2013b). doi:10.1088/0004-637X/ $764 / 2 / 179$

G.E. Brown, C.-H. Lee, The case for case C mass transfer in the galactic evolution of black hole binaries. New Astron. 9, 225-237 (2004). doi:10.1016/j.newast.2003.10.005

D.N. Burrows, P. Romano, A. Falcone, S. Kobayashi, B. Zhang, A. Moretti, P.T. O’Brien, M.R. Goad, S. Campana, K.L. Page, L. Angelini, S. Barthelmy, A.P. Beardmore, M. Capalbi, G. Chincarini, J. Cummings, G. Cusumano, D. Fox, P. Giommi, J.E. Hill, J.A. Kennea, H. Krimm, V. Mangano, F. Marshall, P. Mészáros, D.C. Morris, J.A. Nousek, J.P. Osborne, C. Pagani, M. Perri, G. Tagliaferri, A.A. Wells, S. Woosley, N. Gehrels, Bright X-ray flares in gamma-ray burst afterglows. Science 309, 1833-1835 (2005). doi:10.1126/science. 1116168

N.R. Butler, D. Kocevski, J.S. Bloom, J.L. Curtis, A complete catalog of Swift gamma-ray burst spectra and durations: demise of a physical origin for pre-Swift high-energy correlations. Astrophys. J. 671, 656-677 (2007). doi:10.1086/522492

F. Calura, F. Matteucci, The cosmic evolution of the galaxy luminosity density. Astrophys. J. 596, 734-747 (2003). doi:10.1086/378195

S. Campana, V. Mangano, A.J. Blustin, P. Brown, D.N. Burrows, G. Chincarini, J.R. Cummings, G. Cusumano, M. Della Valle, D. Malesani, P. Mészáros, J.A. Nousek, M. Page, T. Sakamoto, E. Wax- 
man, B. Zhang, Z.G. Dai, N. Gehrels, S. Immler, F.E. Marshall, K.O. Mason, A. Moretti, P.T. O’Brien, J.P. Osborne, K.L. Page, P. Romano, P.W.A. Roming, G. Tagliaferri, L.R. Cominsky, P. Giommi, O. Godet, J.A. Kennea, H. Krimm, L. Angelini, S.D. Barthelmy, P.T. Boyd, D.M. Palmer, A.A. Wells, N.E. White, The association of GRB 060218 with a supernova and the evolution of the shock wave. Nature 442, 1008-1010 (2006). doi:10.1038/nature04892

Z. Cano, A new method for estimating the bolometric properties of Ibc supernovae. Mon. Not. R. Astron. Soc. 434, 1098-1116 (2013). doi:10.1093/mnras/stt1048

Z. Cano, K.G. Johansson Andreas, K. Maeda, A self-consistent analytical magnetar model: the luminosity of $\gamma$-ray burst supernovae is powered by radioactivity. Mon. Not. R. Astron. Soc. 457, 2761-2772 (2016). doi:10.1093/mnras/stw122

Z. Cano, A. de Ugarte Postigo, A. Pozanenko, N. Butler, C.C. Thöne, C. Guidorzi, T. Krühler, J. Gorosabel, P. Jakobsson, G. Leloudas, D. Malesani, J. Hjorth, A. Melandri, C. Mundell, K. Wiersema, P. D’Avanzo, S. Schulze, A. Gomboc, A. Johansson, W. Zheng, D.A. Kann, F. Knust, K. Varela, C.W. Akerlof, J. Bloom, O. Burkhonov, E. Cooke, J.A. de Diego, G. Dhungana, C. Farina, F.V. Ferrante, H.A. Flewelling, O.D. Fox, J. Fynbo, N. Gehrels, L. Georgiev, J.J. González, J. Greiner, T. Güver, O. Hartoog, N. Hatch, M. Jelinek, R. Kehoe, S. Klose, E. Klunko, D. Kopač, A. Kutyrev, Y. Krugly, W.H. Lee, A. Levan, V. Linkov, A. Matkin, N. Minikulov, I. Molotov, J.X. Prochaska, M.G. Richer, C.G. Román-Zúñiga, V. Rumyantsev, R. Sánchez-Ramírez, I. Steele, N.R. Tanvir, A. Volnova, A.M. Watson, D. Xu, F. Yuan, A trio of gamma-ray burst supernovae: GRB 120729A, GRB 130215A/SN 2013ez, and GRB 130831A/SN 2013fu. Astron. Astrophys. 568, 19 (2014). doi:10.1051/ 0004-6361/201423920

G. Cavallo, M.J. Rees, A qualitative study of cosmic fireballs and gamma-ray bursts. Mon. Not. R. Astron. Soc. 183, 359-365 (1978). doi:10.1093/mnras/183.3.359

R. Chapman, N.R. Tanvir, R.S. Priddey, A.J. Levan, How common are long gamma-ray bursts in the local Universe? Mon. Not. R. Astron. Soc. 382, 21-25 (2007). doi:10.1111/j.1745-3933.2007.00381.x

H.-Y. Chen, D.E. Holz, Gamma-ray-burst beaming and gravitational-wave observations. Phys. Rev. Lett. 111(18), 181101 (2013). doi:10.1103/PhysRevLett.111.181101

A. Coleiro, S. Chaty, Distribution of high-mass X-ray binaries in the Milky Way. Astrophys. J. 764, 185 (2013). doi:10.1088/0004-637X/764/2/185

E. Costa, F. Frontera, J. Heise, M. Feroci, J. in't Zand, F. Fiore, M.N. Cinti, D. Dal Fiume, L. Nicastro, M. Orlandini, E. Palazzi, M. Rapisarda\#, G. Zavattini, R. Jager, A. Parmar, A. Owens, S. Molendi, G. Cusumano, M.C. Maccarone, S. Giarrusso, A. Coletta, L.A. Antonelli, P. Giommi, J.M. Muller, L. Piro, R.C. Butler, Discovery of an X-ray afterglow associated with the $\gamma$-ray burst of 28 February 1997. Nature 387, 783-785 (1997). doi:10.1038/42885

P.A. Crowther, Physical properties of Wolf-Rayet stars. Annu. Rev. Astron. Astrophys. 45, 177-219 (2007). doi:10.1146/annurev.astro.45.051806.110615

P.A. Crowther, L. Dessart, D.J. Hillier, J.B. Abbott, A.W. Fullerton, Stellar and wind properties of LMC WC4 stars. A metallicity dependence for Wolf-Rayet mass-loss rates. Astron. Astrophys. 392, 653-669 (2002). doi:10.1051/0004-6361:20020941

P.A. Crowther, S.M. Caballero-Nieves, K.A. Bostroem, J. Maíz Apellániz, F.R.N. Schneider, N.R. Walborn, C.R. Angus, I. Brott, A. Bonanos, A. de Koter, S.E. de Mink, C.J. Evans, G. Gräfener, A. Herrero, I.D. Howarth, N. Langer, D.J. Lennon, J. Puls, H. Sana, J.S. Vink, The R136 star cluster dissected with Hubble Space Telescope/STIS. I. Far-ultraviolet spectroscopic census and the origin of He II $\lambda 1640$ in young star clusters. Mon. Not. R. Astron. Soc. 458, 624-659 (2016). doi:10.1093/mnras/stw273

A. Cucchiara, A.J. Levan, D.B. Fox, N.R. Tanvir, T.N. Ukwatta, E. Berger, T. Krühler, A. Küpcü Yoldaş, X.F. Wu, K. Toma, J. Greiner, F.E. Olivares, A. Rowlinson, L. Amati, T. Sakamoto, K. Roth, A. Stephens, A. Fritz, J.P.U. Fynbo, J. Hjorth, D. Malesani, P. Jakobsson, K. Wiersema, P.T. O’Brien, A.M. Soderberg, R.J. Foley, A.S. Fruchter, J. Rhoads, R.E. Rutledge, B.P. Schmidt, M.A. Dopita, P. Podsiadlowski, R. Willingale, C. Wolf, S.R. Kulkarni, P. D’Avanzo, A photometric redshift of z $\sim 9.4$ for GRB 090429B. Astrophys. J. 736, 7 (2011). doi:10.1088/0004-637X/736/1/7

M.B. Davies, A.J. Levan, A.R. King, The ultimate outcome of black hole-neutron star mergers. Mon. Not. R. Astron. Soc. 356, 54-58 (2005). doi:10.1111/j.1365-2966.2004.08423.x

M.B. Davies, A. King, S. Rosswog, G. Wynn, Gamma-ray bursts, supernova kicks, and gravitational radiation. Astrophys. J. Lett. 579, 63-66 (2002). doi:10.1086/345288

R. de Grijs, C. Li, Y. Zheng, L. Deng, Y. Hu, M.B.N. Kouwenhoven, Gravitational conundrum? Dynamical mass segregation versus disruption of binary stars in dense stellar systems. Astrophys. J. 765, 4 (2013). doi:10.1088/0004-637X/765/1/4

S.E. de Mink, N. Langer, R.G. Izzard, H. Sana, A. de Koter, The rotation rates of massive stars: the role of binary interaction through tides, mass transfer, and mergers. Astrophys. J. 764, 166 (2013). doi:10.1088/ $0004-637 \mathrm{X} / 764 / 2 / 166$ 
A. de Ugarte Postigo, A.J. Castro-Tirado, S. Guziy, J. Gorosabel, G. Jóhannesson, M.A. Aloy, S. McBreen, D.Q. Lamb, N. Benitez, M. Jelínek, S.B. Pandey, D. Coe, M.D. Pérez-Ramírez, F.J. Aceituno, M. Alises, J.A. Acosta-Pulido, G. Gómez, R. López, T.Q. Donaghy, Y.E. Nakagawa, T. Sakamoto, G.R. Ricker, F.R. Hearty, M. Bayliss, G. Gyuk, GRB 060121: implications of a short-/intermediate-duration $\gamma$-ray burst at high redshift. Astrophys. J. Lett. 648, 83-87 (2006). doi:10.1086/507868

A. de Ugarte Postigo, I. Horváth, P. Veres, Z. Bagoly, D.A. Kann, C.C. Thöne, L.G. Balazs, P. D’Avanzo, M.A. Aloy, S. Foley, S. Campana, J. Mao, P. Jakobsson, S. Covino, J.P.U. Fynbo, J. Gorosabel, A.J. Castro-Tirado, L. Amati, M. Nardini, Searching for differences in Swift's intermediate GRBs. Astron. Astrophys. 525, 109 (2011). doi:10.1051/0004-6361/201015261

A. de Ugarte Postigo, C.C. Thöne, A. Rowlinson, R. García-Benito, A.J. Levan, J. Gorosabel, P. Goldoni, S. Schulze, T. Zafar, K. Wiersema, R. Sánchez-Ramírez, A. Melandri, P. D’Avanzo, S. Oates, V. D’Elia, M. De Pasquale, T. Krühler, A.J. van der Horst, D. Xu, D. Watson, S. Piranomonte, S.D. Vergani, B. Milvang-Jensen, L. Kaper, D. Malesani, J.P.U. Fynbo, Z. Cano, S. Covino, H. Flores, S. Greiss, F. Hammer, O.E. Hartoog, S. Hellmich, C. Heuser, J. Hjorth, P. Jakobsson, S. Mottola, M. Sparre, J. Sollerman, G. Tagliaferri, N.R. Tanvir, M. Vestergaard, R.A.M.J. Wijers, Spectroscopy of the shorthard GRB 130603B. The host galaxy and environment of a compact object merger. Astron. Astrophys. 563, 62 (2014). doi:10.1051/0004-6361/201322985

M. Della Valle, G. Chincarini, N. Panagia, G. Tagliaferri, D. Malesani, V. Testa, D. Fugazza, S. Campana, S. Covino, V. Mangano, L.A. Antonelli, P. D’Avanzo, K. Hurley, I.F. Mirabel, L.J. Pellizza, S. Piranomonte, L. Stella, An enigmatic long-lasting $\gamma$-ray burst not accompanied by a bright supernova. Nature 444, 1050-1052 (2006). doi:10.1038/nature05374

J. Deng, N. Tominaga, P.A. Mazzali, K. Maeda, K. Nomoto, On the light curve and spectrum of SN 2003dh separated from the optical afterglow of GRB 030329. Astrophys. J. 624, 898-905 (2005). doi:10.1086/ 429284

R.G. Detmers, N. Langer, P. Podsiadlowski, R.G. Izzard, Gamma-ray bursts from tidally spun-up Wolf-Rayet stars? Astron. Astrophys. 484, 831-839 (2008). doi:10.1051/0004-6361:200809371

A. Dietz, N. Fotopoulos, L. Singer, C. Cutler, Outlook for detection of GW inspirals by GRBtriggered searches in the advanced detector era. Phys. Rev. D 87(6), 064033 (2013). doi:10.1103/ PhysRevD.87.064033

M.A. Dopita, L.J. Kewley, R.S. Sutherland, D.C. Nicholls, Chemical abundances in high-redshift galaxies: a powerful new emission line diagnostic. Astrophys. Space Sci. 361, 61 (2016). doi:10.1007/ s10509-016-2657-8

A.S. Eddington, The Internal Constitution of the Stars (1926)

J.J. Eldridge, E.R. Stanway, Spectral population synthesis including massive binaries. Mon. Not. R. Astron. Soc. 400, 1019-1028 (2009). doi:10.1111/j.1365-2966.2009.15514.x

R.A.W. Elson, S. Sigurdsson, M. Davies, J. Hurley, G. Gilmore, The binary star population of the young cluster NGC 1818 in the Large Magellanic Cloud. Mon. Not. R. Astron. Soc. 300, 857-862 (1998). doi:10. 1046/j.1365-8711.1998.01948.x

C.J. Evans, W.D. Taylor, V. Hénault-Brunet, H. Sana, A. de Koter, S. Simón-Díaz, G. Carraro, T. Bagnoli, N. Bastian, J.M. Bestenlehner, A.Z. Bonanos, E. Bressert, I. Brott, M.A. Campbell, M. Cantiello, J.S. Clark, E. Costa, P.A. Crowther, S.E. de Mink, E. Doran, P.L. Dufton, P.R. Dunstall, K. Friedrich, M. Garcia, M. Gieles, G. Gräfener, A. Herrero, I.D. Howarth, R.G. Izzard, N. Langer, D.J. Lennon, J. Maíz Apellániz, N. Markova, F. Najarro, J. Puls, O.H. Ramirez, C. Sabín-Sanjulián, S.J. Smartt, V.E. Stroud, J.T. van Loon, J.S. Vink, N.R. Walborn, The VLT-FLAMES Tarantula Survey. I. Introduction and observational overview. Astron. Astrophys. 530, 108 (2011). doi:10.1051/0004-6361/ 201116782

R.J. Foley, P.J. Challis, R. Chornock, M. Ganeshalingam, W. Li, G.H. Marion, N.I. Morrell, G. Pignata, M.D. Stritzinger, J.M. Silverman, X. Wang, J.P. Anderson, A.V. Filippenko, W.L. Freedman, M. Hamuy, S.W. Jha, R.P. Kirshner, C. McCully, S.E. Persson, M.M. Phillips, D.E. Reichart, A.M. Soderberg, Type Iax supernovae: a new class of stellar explosion. Astrophys. J. 767, 57 (2013). doi:10.1088/ 0004-637X/767/1/57

W. Fong, E. Berger, The locations of short gamma-ray bursts as evidence for compact object binary progenitors. Astrophys. J. 776, 18 (2013). doi:10.1088/0004-637X/776/1/18

W. Fong, E. Berger, D.B. Fox, Hubble space telescope observations of short gamma-ray burst host galaxies: morphologies, offsets, and local environments. Astrophys. J. 708, 9-25 (2010). doi:10.1088/ 0004-637X/708/1/9

W-f. Fong, B.D. Metzger, E. Berger, F. Ozel, Radio Constraints on Long-Lived Magnetar Remnants in Short Gamma-Ray Bursts. ArXiv e-prints (2016)

W. Fong, E. Berger, R. Chornock, R. Margutti, A.J. Levan, N.R. Tanvir, R.L. Tunnicliffe, I. Czekala, D.B. Fox, D.A. Perley, S.B. Cenko, B.A. Zauderer, T. Laskar, S.E. Persson, A.J. Monson, D.D. Kelson, C. Birk, 
D. Murphy, M. Servillat, G. Anglada, Demographics of the galaxies hosting short-duration gamma-ray bursts. Astrophys. J. 769, 56 (2013). doi:10.1088/0004-637X/769/1/56

W. Fong, E. Berger, B.D. Metzger, R. Margutti, R. Chornock, G. Migliori, R.J. Foley, B.A. Zauderer, R. Lunnan, T. Laskar, S.J. Desch, K.J. Meech, S. Sonnett, C. Dickey, A. Hedlund, P. Harding, Short GRB 130603B: discovery of a jet break in the optical and radio afterglows, and a mysterious late-time X-ray excess. Astrophys. J. 780, 118 (2014). doi:10.1088/0004-637X/780/2/118

D.B. Fox, D.A. Frail, P.A. Price, S.R. Kulkarni, E. Berger, T. Piran, A.M. Soderberg, S.B. Cenko, P.B. Cameron, A. Gal-Yam, M.M. Kasliwal, D.-S. Moon, F.A. Harrison, E. Nakar, B.P. Schmidt, B. Penprase, R.A. Chevalier, P. Kumar, K. Roth, D. Watson, B.L. Lee, S. Shectman, M.M. Phillips, M. Roth, P.J. McCarthy, M. Rauch, L. Cowie, B.A. Peterson, J. Rich, N. Kawai, K. Aoki, G. Kosugi, T. Totani, H.-S. Park, A. MacFadyen, K.C. Hurley, The afterglow of GRB 050709 and the nature of the short-hard $\gamma$-ray bursts. Nature 437, 845-850 (2005). doi:10.1038/nature04189

D.A. Frail, S.R. Kulkarni, L. Nicastro, M. Feroci, G.B. Taylor, The radio afterglow from the $\gamma$-ray burst of 8 May 1997. Nature 389, 261-263 (1997). doi:10.1038/38451

D.A. Frail, S.R. Kulkarni, R. Sari, S.G. Djorgovski, J.S. Bloom, T.J. Galama, D.E. Reichart, E. Berger, F.A. Harrison, P.A. Price, S.A. Yost, A. Diercks, R.W. Goodrich, F. Chaffee, Beaming in gamma-ray bursts: evidence for a standard energy reservoir. Astrophys. J. Lett. 562, 55-58 (2001). doi:10.1086/ 338119

A.S. Fruchter, A.J. Levan, L. Strolger, P.M. Vreeswijk, S.E. Thorsett, D. Bersier, I. Burud, J.M. Castro Cerón, A.J. Castro-Tirado, C. Conselice, T. Dahlen, H.C. Ferguson, J.P.U. Fynbo, P.M. Garnavich, R.A. Gibbons, J. Gorosabel, T.R. Gull, J. Hjorth, S.T. Holland, C. Kouveliotou, Z. Levay, M. Livio, M.R. Metzger, P.E. Nugent, L. Petro, E. Pian, J.E. Rhoads, A.G. Riess, K.C. Sahu, A. Smette, N.R. Tanvir, R.A.M.J. Wijers, S.E. Woosley, Long $\gamma$-ray bursts and core-collapse supernovae have different environments. Nature 441, 463-468 (2006). doi:10.1038/nature04787

C.L. Fryer, A.L. Hungerford, P.A. Young, Light-curve calculations of supernovae from fallback gamma-ray bursts. Astrophys. J. Lett. 662, 55-58 (2007). doi:10.1086/519523

C.L. Fryer, S.E. Woosley, D.H. Hartmann, Formation rates of black hole accretion disk gamma-ray bursts. Astrophys. J. 526, 152-177 (1999). doi:10.1086/307992

C.L. Fryer, S.E. Woosley, A. Heger, Pair-instability supernovae, gravity waves, and gamma-ray transients. Astrophys. J. 550, 372-382 (2001). doi:10.1086/319719

J.P.U. Fynbo, D. Watson, C.C. Thöne, J. Sollerman, J.S. Bloom, T.M. Davis, J. Hjorth, P. Jakobsson, U.G. Jørgensen, J.F. Graham, A.S. Fruchter, D. Bersier, L. Kewley, A. Cassan, J.M. Castro Cerón, S. Foley, J. Gorosabel, T.C. Hinse, K.D. Horne, B.L. Jensen, S. Klose, D. Kocevski, J.-B. Marquette, D. Perley, E. Ramirez-Ruiz, M.D. Stritzinger, P.M. Vreeswijk, R.A.M. Wijers, K.G. Woller, D. Xu, M. Zub, No supernovae associated with two long-duration $\gamma$-ray bursts. Nature 444, 1047-1049 (2006). doi:10.1038/ nature 05375

J.P.U. Fynbo, P. Jakobsson, J.X. Prochaska, D. Malesani, C. Ledoux, A. de Ugarte Postigo, M. Nardini, P.M. Vreeswijk, K. Wiersema, J. Hjorth, J. Sollerman, H.-W. Chen, C.C. Thöne, G. Björnsson, J.S. Bloom, A.J. Castro-Tirado, L. Christensen, A. De Cia, A.S. Fruchter, J. Gorosabel, J.F. Graham, A.O. Jaunsen, B.L. Jensen, D.A. Kann, C. Kouveliotou, A.J. Levan, J. Maund, N. Masetti, B. MilvangJensen, E. Palazzi, D.A. Perley, E. Pian, E. Rol, P. Schady, R.L.C. Starling, N.R. Tanvir, D.J. Watson, D. Xu, T. Augusteijn, F. Grundahl, J. Telting, P.-O. Quirion, Low-resolution spectroscopy of gamma-ray burst optical afterglows: biases in the Swift sample and characterization of the absorbers. Astrophys. J. Suppl. Ser. 185, 526-573 (2009). doi:10.1088/0067-0049/185/2/526

A. Gal-Yam, Luminous supernovae. Science 337, 927 (2012). doi:10.1126/science. 1203601

A. Gal-Yam, D.B. Fox, P.A. Price, E.O. Ofek, M.R. Davis, D.C. Leonard, A.M. Soderberg, B.P. Schmidt, K.M. Lewis, B.A. Peterson, S.R. Kulkarni, E. Berger, S.B. Cenko, R. Sari, K. Sharon, D. Frail, D.-S. Moon, P.J. Brown, A. Cucchiara, F. Harrison, T. Piran, S.E. Persson, P.J. McCarthy, B.E. Penprase, R.A. Chevalier, A.I. MacFadyen, A novel explosive process is required for the $\gamma$-ray burst GRB 060614. Nature 444, 1053-1055 (2006). doi:10.1038/nature05373

A. Gal-Yam, P. Mazzali, E.O. Ofek, P.E. Nugent, S.R. Kulkarni, M.M. Kasliwal, R.M. Quimby, A.V. Filippenko, S.B. Cenko, R. Chornock, R. Waldman, D. Kasen, M. Sullivan, E.C. Beshore, A.J. Drake, R.C. Thomas, J.S. Bloom, D. Poznanski, A.A. Miller, R.J. Foley, J.M. Silverman, I. Arcavi, R.S. Ellis, J. Deng, Supernova 2007bi as a pair-instability explosion. Nature 462, 624-627 (2009). doi:10.1038/ nature 08579

T.J. Galama, N. Tanvir, P.M. Vreeswijk, R.A.M.J. Wijers, P.J. Groot, E. Rol, J. van Paradijs, C. Kouveliotou, A.S. Fruchter, N. Masetti, H. Pedersen, B. Margon, E.W. Deutsch, M. Metzger, L. Armus, S. Klose, B. Stecklum, Evidence for a supernova in reanalyzed optical and near-infrared images of GRB 970228. Astrophys. J. 536, 185-194 (2000). doi:10.1086/308909

P.M. Garnavich, K.Z. Stanek, L. Wyrzykowski, L. Infante, E. Bendek, D. Bersier, S.T. Holland, S. Jha, T. Matheson, R.P. Kirshner, K. Krisciunas, M.M. Phillips, R.G. Carlberg, Discovery of the low-redshift 
optical afterglow of GRB 011121 and its progenitor supernova SN 2001ke. Astrophys. J. 582, 924-932 (2003). doi:10.1086/344785

N. Gehrels, C.L. Sarazin, P.T. O’Brien, B. Zhang, L. Barbier, S.D. Barthelmy, A. Blustin, D.N. Burrows, J. Cannizzo, J.R. Cummings, M. Goad, S.T. Holland, C.P. Hurkett, J.A. Kennea, A. Levan, C.B. Markwardt, K.O. Mason, P. Meszaros, M. Page, D.M. Palmer, E. Rol, T. Sakamoto, R. Willingale, L. Angelini, A. Beardmore, P.T. Boyd, A. Breeveld, S. Campana, M.M. Chester, G. Chincarini, L.R. Cominsky, G. Cusumano, M. de Pasquale, E.E. Fenimore, P. Giommi, C. Gronwall, D. Grupe, J.E. Hill, D. Hinshaw, J. Hjorth, D. Hullinger, K.C. Hurley, S. Klose, S. Kobayashi, C. Kouveliotou, H.A. Krimm, V. Mangano, F.E. Marshall, K. McGowan, A. Moretti, R.F. Mushotzky, K. Nakazawa, J.P. Norris, J.A. Nousek, J.P. Osborne, K. Page, A.M. Parsons, S. Patel, M. Perri, T. Poole, P. Romano, P.W.A. Roming, S. Rosen, G. Sato, P. Schady, A.P. Smale, J. Sollerman, R. Starling, M. Still, M. Suzuki, G. Tagliaferri, T. Takahashi, M. Tashiro, J. Tueller, A.A. Wells, N.E. White, R.A.M.J. Wijers, A short $\gamma$-ray burst apparently associated with an elliptical galaxy at redshift $\mathrm{z}=0.225$. Nature 437, 851-854 (2005). doi:10.1038/nature04142

N. Gehrels, J.P. Norris, S.D. Barthelmy, J. Granot, Y. Kaneko, C. Kouveliotou, C.B. Markwardt, P. Mészáros, E. Nakar, J.A. Nousek, P.T. O’Brien, M. Page, D.M. Palmer, A.M. Parsons, P.W.A. Roming, T. Sakamoto, C.L. Sarazin, P. Schady, M. Stamatikos, S.E. Woosley, A new $\gamma$-ray burst classification scheme from GRB060614. Nature 444, 1044-1046 (2006). doi:10.1038/nature05376

N. Gehrels, S.D. Barthelmy, D.N. Burrows, J.K. Cannizzo, G. Chincarini, E. Fenimore, C. Kouveliotou, P. O'Brien, D.M. Palmer, J. Racusin, P.W.A. Roming, T. Sakamoto, J. Tueller, R.A.M.J. Wijers, B. Zhang, Correlations of prompt and afterglow emission in Swift long and short gamma-ray bursts. Astrophys. J. 689, 1161-1172 (2008). doi:10.1086/592766

A.M. Geller, R. de Grijs, C. Li, J.R. Hurley, Consequences of dynamical disruption and mass segregation for the binary frequencies of star clusters. Astrophys. J. 779, 30 (2013). doi:10.1088/0004-637X/779/1/30

A.M. Geller, R. de Grijs, C. Li, J.R. Hurley, Different dynamical ages for the two young and coeval LMC star clusters, NGC 1805 and NGC 1818, imprinted on their binary populations. Astrophys. J. 805, 11 (2015). doi:10.1088/0004-637X/805/1/11

B. Gendre, G. Stratta, J.L. Atteia, S. Basa, M. Boër, D.M. Coward, S. Cutini, V. D’Elia, E.J. Howell, A. Klotz, L. Piro, The ultra-long gamma-ray burst 111209A: the collapse of a blue supergiant? Astrophys. J. 766, 30 (2013). doi:10.1088/0004-637X/766/1/30

B.P. Gompertz, A.J. van der Horst, P.T. O’Brien, G.A. Wynn, K. Wiersema, Broad-band modelling of short gamma-ray bursts with energy injection from magnetar spin-down and its implications for radio detectability. Mon. Not. R. Astron. Soc. 448, 629-641 (2015). doi:10.1093/mnras/stu2752

J. Gorosabel, J.P.U. Fynbo, A. Fruchter, A. Levan, J. Hjorth, P. Nugent, A.J. Castro-Tirado, J.M. Castro Cerón, J. Rhoads, D. Bersier, I. Burud, A possible bright blue supernova in the afterglow of GRB 020305. Astron. Astrophys. 437, 411-418 (2005). doi:10.1051/0004-6361:20052783

J.F. Graham, A.S. Fruchter, The metal aversion of long-duration gamma-ray bursts. Astrophys. J. 774, 119 (2013). doi:10.1088/0004-637X/774/2/119

J.F. Graham, A.S. Fruchter, The Relative Rate of LGRB Formation as a Function of Metallicity. ArXiv e-prints (2015a)

J.F. Graham, P. Schady, The Absolute Rate of LGRB Formation. ArXiv e-prints (2015b)

J.F. Graham, A.S. Fruchter, A.J. Levan, A. Melandri, L.J. Kewley, E.M. Levesque, M. Nysewander, N.R. Tanvir, T. Dahlen, D. Bersier, K. Wiersema, D.G. Bonfield, A. Martinez-Sansigre, GRB 070714B: discovery of the highest spectroscopically confirmed short burst redshift. Astrophys. J. 698, 1620-1629 (2009). doi:10.1088/0004-637X/698/2/1620

J. Greiner, P.A. Mazzali, D.A. Kann, T. Krühler, E. Pian, S. Prentice, F. Olivares E., A. Rossi, S. Klose, S. Taubenberger, F. Knust, P.M.J. Afonso, C. Ashall, J. Bolmer, C. Delvaux, R. Diehl, J. Elliott, R. Filgas, J.P.U. Fynbo, J.F. Graham, A.N. Guelbenzu, S. Kobayashi, G. Leloudas, S. Savaglio, P. Schady, S. Schmidl, T. Schweyer, V. Sudilovsky, M. Tanga, A.C. Updike, H. van Eerten, K. Varela, A very luminous magnetar-powered supernova associated with an ultra-long $\gamma$-ray burst. Nature 523, 189-192 (2015a). doi:10.1038/nature14579

R. Hainich, D. Pasemann, H. Todt, T. Shenar, A. Sander, W.-R. Hamann, Wolf-Rayet stars in the Small Magellanic Cloud. I. Analysis of the single WN stars. Astron. Astrophys. 581, 21 (2015b). doi:10.1051/ 0004-6361/201526241

F. Hammer, H. Flores, D. Schaerer, M. Dessauges-Zavadsky, E. Le Floc'h, M. Puech, Detection of WolfRayet stars in host galaxies of gamma-ray bursts (GRBs): are GRBs produced by runaway massive stars ejected from high stellar density regions? Astron. Astrophys. 454, 103-111 (2006). doi:10.1051/ 0004-6361:20064823

A. Heger, S.E. Woosley, H.C. Spruit, Presupernova evolution of differentially rotating massive stars including magnetic fields. Astrophys. J. 626, 350-363 (2005). doi:10.1086/429868 
D.C. Heggie, Binary evolution in stellar dynamics. Mon. Not. R. Astron. Soc. 173, 729-787 (1975). doi:10. 1093/mnras/173.3.729

J. Hjorth, J. Sollerman, P. Møller, J.P.U. Fynbo, S.E. Woosley, C. Kouveliotou, N.R. Tanvir, J. Greiner, M.I. Andersen, A.J. Castro-Tirado, J.M. Castro Cerón, A.S. Fruchter, J. Gorosabel, P. Jakobsson, L. Kaper, S. Klose, N. Masetti, H. Pedersen, K. Pedersen, E. Pian, E. Palazzi, J.E. Rhoads, E. Rol, E.P.J. van den Heuvel, P.M. Vreeswijk, D. Watson, R.A.M.J. Wijers, A very energetic supernova associated with the $\gamma$-ray burst of 29 March 2003. Nature 423, 847-850 (2003). doi:10.1038/nature01750

J. Hjorth, J. Sollerman, J. Gorosabel, J. Granot, S. Klose, C. Kouveliotou, J. Melinder, E. Ramirez-Ruiz, R. Starling, B. Thomsen, M.I. Andersen, J.P.U. Fynbo, B.L. Jensen, P.M. Vreeswijk, J.M. Castro Cerón, P. Jakobsson, A. Levan, K. Pedersen, J.E. Rhoads, N.R. Tanvir, D. Watson, R.A.M.J. Wijers, GRB 050509B: constraints on short gamma-ray burst models. Astrophys. J. Lett. 630, 117-120 (2005a). doi: $10.1086 / 491733$

J. Hjorth, D. Watson, J.P.U. Fynbo, P.A. Price, B.L. Jensen, U.G. Jørgensen, D. Kubas, J. Gorosabel, P. Jakobsson, J. Sollerman, K. Pedersen, C. Kouveliotou, The optical afterglow of the short $\gamma$-ray burst GRB 050709. Nature 437, 859-861 (2005b). doi:10.1038/nature04174

P. Höflich, J.C. Wheeler, L. Wang, Aspherical explosion models for SN 1998BW/GRB-980425. Astrophys. J. 521, 179-189 (1999). doi:10.1086/307521

Y. Hu, L. Deng, R. de Grijs, Q. Liu, S.P. Goodwin, The binary fraction of the young cluster NGC 1818 in the Large Magellanic Cloud. Astrophys. J. 724, 649-656 (2010). doi:10.1088/0004-637X/724/1/649

K. Hurley, S.E. Boggs, D.M. Smith, R.C. Duncan, R. Lin, A. Zoglauer, S. Krucker, G. Hurford, H. Hudson, C. Wigger, W. Hajdas, C. Thompson, I. Mitrofanov, A. Sanin, W. Boynton, C. Fellows, A. von Kienlin, G. Lichti, A. Rau, T. Cline, An exceptionally bright flare from SGR 1806-20 and the origins of shortduration $\gamma$-ray bursts. Nature 434, 1098-1103 (2005). doi:10.1038/nature03519

R.G. Izzard, E. Ramirez-Ruiz, C.A. Tout, Formation rates of core-collapse supernovae and gamma-ray bursts. Mon. Not. R. Astron. Soc. 348, 1215-1228 (2004). doi:10.1111/j.1365-2966.2004.07436.x

P.A. James, J.P. Anderson, The H $\alpha$ galaxy survey. III. Constraints on supernova progenitors from spatial correlations with $\mathrm{H} \alpha$ emission. Astron. Astrophys. 453, 57-65 (2006). doi:10.1051/0004-6361:20054509

J. Japelj, S.D. Vergani, R. Salvaterra, L.K. Hunt, F. Mannucci, Taking stock of SLSN and LGRB host galaxy comparison using a complete sample of LGRBs. ArXiv e-prints (2016)

Z.-P. Jin, X. Li, Z. Cano, S. Covino, Y.-Z. Fan, D.-M. Wei, The light curve of the macronova associated with the long-short burst GRB 060614. Astrophys. J. Lett. 811, 22 (2015). doi:10.1088/2041-8205/ $811 / 2 / \mathrm{L} 22$

D.A. Kann, S. Klose, B. Zhang, D. Malesani, E. Nakar, A. Pozanenko, A.C. Wilson, N.R. Butler, P. Jakobsson, S. Schulze, M. Andreev, L.A. Antonelli, I.F. Bikmaev, V. Biryukov, M. Böttcher, R.A. Burenin, J.M. Castro Cerón, A.J. Castro-Tirado, G. Chincarini, B.E. Cobb, S. Covino, P. D'Avanzo, V. D'Elia, M. Della Valle, A. de Ugarte Postigo, Y. Efimov, P. Ferrero, D. Fugazza, J.P.U. Fynbo, M. Gålfalk, F. Grundahl, J. Gorosabel, S. Gupta, S. Guziy, B. Hafizov, J. Hjorth, K. Holhjem, M. Ibrahimov, M. Im, G.L. Israel, M. Jelínek, B.L. Jensen, R. Karimov, I.M. Khamitov, Ü. Kiziloğlu, E. Klunko, P. Kubánek, A.S. Kutyrev, P. Laursen, A.J. Levan, F. Mannucci, C.M. Martin, A. Mescheryakov, N. Mirabal, J.P. Norris, J.-E. Ovaldsen, D. Paraficz, E. Pavlenko, S. Piranomonte, A. Rossi, V. Rumyantsev, R. Salinas, A. Sergeev, D. Sharapov, J. Sollerman, B. Stecklum, L. Stella, G. Tagliaferri, N.R. Tanvir, J. Telting, V. Testa, A.C. Updike, A. Volnova, D. Watson, K. Wiersema, D. Xu, The afterglows of Swift-era gammaray bursts. I. Comparing pre-Swift and Swift-era long/soft (type II) GRB optical afterglows. Astrophys. J. 720, 1513-1558 (2010). doi:10.1088/0004-637X/720/2/1513

D.A. Kann, P. Schady, F. Olivares E, S. Klose, A. Rossi, D.A. Perley, T. Krühler, J. Greiner, A. Nicuesa Guelbenzu, J. Elliott, F. Knust, R. Filgas, E. Pian, P. Mazzali, J.P.U. Fynbo, G. Leloudas, P.M.J. Afonso, C. Delvaux, J.F. Graham, A. Rau, S. Schmidl, S. Schulze, M. Tanga, A.C. Updike, K. Varela, Highly Luminous Supernovae associated with Gamma-Ray Bursts I: GRB 111209A/SN 2011kl in the Context of Stripped-Envelope and Superluminous Supernovae. ArXiv e-prints (2016)

D. Kasen, L. Bildsten, Supernova light curves powered by young magnetars. Astrophys. J. 717, 245-249 (2010). doi:10.1088/0004-637X/717/1/245

M.M. Kasliwal, S.R. Kulkarni, A. Gal-Yam, P.E. Nugent, M. Sullivan, L. Bildsten, O. Yaron, H.B. Perets, I. Arcavi, S. Ben-Ami, V.B. Bhalerao, J.S. Bloom, S.B. Cenko, A.V. Filippenko, D.A. Frail, M. Ganeshalingam, A. Horesh, D.A. Howell, N.M. Law, D.C. Leonard, W. Li, E.O. Ofek, D. Polishook, D. Poznanski, R.M. Quimby, J.M. Silverman, A. Sternberg, D. Xu, Calcium-rich gap transients in the remote outskirts of galaxies. Astrophys. J. 755, 161 (2012). doi:10.1088/0004-637X/755/2/161

P.L. Kelly, R.P. Kirshner, Core-collapse supernovae and host galaxy stellar populations. Astrophys. J. 759, 107 (2012). doi:10.1088/0004-637X/759/2/107

P.L. Kelly, R.P. Kirshner, M. Pahre, Long $\gamma$-ray bursts and type Ic core-collapse supernovae have similar locations in hosts. Astrophys. J. 687, 1201-1207 (2008). doi:10.1086/591925 
P.L. Kelly, A.V. Filippenko, O.D. Fox, W. Zheng, K.I. Clubb, Evidence that gamma-ray burst 130702A exploded in a dwarf satellite of a massive galaxy. Astrophys. J. Lett. 775, 5 (2013). doi:10.1088/2041-8205/ 775/1/L5

S. Kisaka, K. Ioka, T. Nakamura, Isotropic detectable X-ray counterparts to gravitational waves from neutron star binary mergers. Astrophys. J. Lett. 809, 8 (2015). doi:10.1088/2041-8205/809/1/L8

S. Kisaka, K. Ioka, E. Nakar, X-ray-powered macronovae. Astrophys. J. 818, 104 (2016). doi:10.3847/ 0004-637X/818/2/104

R.W. Klebesadel, I.B. Strong, R.A. Olson, Observations of gamma-ray bursts of cosmic origin. Astrophys. J. Lett. 182, 85 (1973). doi:10.1086/181225

H.A. Kobulnicky, D.C. Kiminki, M.J. Lundquist, J. Burke, J. Chapman, E. Keller, K. Lester, E.K. Rolen, E. Topel, A. Bhattacharjee, R.A. Smullen, C.A. Vargas Álvarez, J.C. Runnoe, D.A. Dale, M.M. Brotherton, Toward complete statistics of massive binary stars: penultimate results from the Cygnus OB2 radial velocity survey. Astrophys. J. Suppl. Ser. 213, 34 (2014). doi:10.1088/0067-0049/213/2/34

C.S. Kochanek, Failed supernovae explain the compact remnant mass function. Astrophys. J. 785, 28 (2014). doi:10.1088/0004-637X/785/1/28

C.S. Kochanek, J.F. Beacom, M.D. Kistler, J.L. Prieto, K.Z. Stanek, T.A. Thompson, H. Yüksel, A survey about nothing: monitoring a million supergiants for failed supernovae. Astrophys. J. 684, 1336-1342 (2008). doi:10.1086/590053

C. Kouveliotou, C.A. Meegan, G.J. Fishman, N.P. Bhat, M.S. Briggs, T.M. Koshut, W.S. Paciesas, G.N. Pendleton, Identification of two classes of gamma-ray bursts. Astrophys. J. Lett. 413, 101-104 (1993). doi:10.1086/186969

C. Kouveliotou, S.E. Woosley, S.K. Patel, A. Levan, R. Blandford, E. Ramirez-Ruiz, R.A.M.J. Wijers, M.C. Weisskopf, A. Tennant, E. Pian, P. Giommi, Chandra observations of the X-ray environs of SN 1998bw/GRB 980425. Astrophys. J. 608, 872-882 (2004). doi:10.1086/420878

M.B.N. Kouwenhoven, A.G.A. Brown, H. Zinnecker, L. Kaper, S.F. Portegies Zwart, The primordial binary population. I. A near-infrared adaptive optics search for close visual companions to A star members of Scorpius OB2. Astron. Astrophys. 430, 137-154 (2005). doi:10.1051/0004-6361:20048124

P. Kroupa, On the variation of the initial mass function. Mon. Not. R. Astron. Soc. 322, 231-246 (2001). doi:10.1046/j.1365-8711.2001.04022.x

T. Krühler, D. Malesani, J.P.U. Fynbo, O.E. Hartoog, J. Hjorth, P. Jakobsson, D.A. Perley, A. Rossi, P. Schady, S. Schulze, N.R. Tanvir, S.D. Vergani, K. Wiersema, P.M.J. Afonso, J. Bolmer, Z. Cano, S. Covino, V. D’Elia, A. de Ugarte Postigo, R. Filgas, M. Friis, J.F. Graham, J. Greiner, P. Goldoni, A. Gomboc, F. Hammer, J. Japelj, D.A. Kann, L. Kaper, S. Klose, A.J. Levan, G. Leloudas, B. Milvang-Jensen, A. Nicuesa Guelbenzu, E. Palazzi, E. Pian, S. Piranomonte, R. Sánchez-Ramírez, S. Savaglio, J. Selsing, G. Tagliaferri, P.M. Vreeswijk, D.J. Watson, D. Xu, GRB hosts through cosmic time. VLT/X-Shooter emission-line spectroscopy of $96 \gamma$-ray-burst-selected galaxies at $0.1<\mathrm{z}<3$.6. Astron. Astrophys. 581, 125 (2015). doi:10.1051/0004-6361/201425561

J.B. Lamb, M.S. Oey, D.M. Segura-Cox, A.S. Graus, D.C. Kiminki, J.B. Golden-Marx, J.W. Parker, The runaways and isolated O-type star spectroscopic survey of the SMC (RIOTS4). Astrophys. J. 817, 113 (2016). doi:10.3847/0004-637X/817/2/113

N. Langer, Coupled mass and angular momentum loss of massive main sequence stars. Astron. Astrophys. 329, 551-558 (1998)

N. Langer, C.A. Norman, On the collapsar model of long gamma-ray bursts: constraints from cosmic metallicity evolution. Astrophys. J. Lett. 638, 63-66 (2006). doi:10.1086/500363

J. Larsson, A.J. Levan, M.B. Davies, A.S. Fruchter, A new constraint for gamma-ray burst progenitor mass. Mon. Not. R. Astron. Soc. 376, 1285-1290 (2007). doi:10.1111/j.1365-2966.2007.11523.X

C.-H. Lee, G.E. Brown, R.A.M.J. Wijers, Discovery of a black hole mass-period correlation in soft X-ray transients and its implication for gamma-ray burst and hypernova mechanisms. Astrophys. J. 575, 9961006 (2002). doi:10.1086/341349

W.-H. Lei, B. Zhang, E.-W. Liang, Hyperaccreting black hole as gamma-ray burst central engine. I. Baryon loading in gamma-ray burst jets. Astrophys. J. 765, 125 (2013). doi:10.1088/0004-637X/765/2/125

C.N. Leibler, E. Berger, The stellar ages and masses of short gamma-ray burst host galaxies: investigating the progenitor delay time distribution and the role of mass and star formation in the short gamma-ray burst rate. Astrophys. J. 725, 1202-1214 (2010). doi:10.1088/0004-637X/725/1/1202

G. Leloudas, J. Sollerman, A.J. Levan, J.P.U. Fynbo, D. Malesani, J.R. Maund, Do Wolf-Rayet stars have similar locations in hosts as type Ib/c supernovae and long gamma-ray bursts? Astron. Astrophys. 518, 29 (2010). doi:10.1051/0004-6361/200913753

G. Leloudas, S. Schulze, T. Krühler, J. Gorosabel, L. Christensen, A. Mehner, A. de Ugarte Postigo, R. Amorín, C.C. Thöne, J.P. Anderson, F.E. Bauer, A. Gallazzi, K.G. Hełminiak, J. Hjorth, E. Ibar, D. Malesani, N. Morell, J. Vinko, J.C. Wheeler, Spectroscopy of superluminous supernova host galax- 
ies. A preference of hydrogen-poor events for extreme emission line galaxies. Mon. Not. R. Astron. Soc. 449, 917-932 (2015). doi:10.1093/mnras/stv320

A.J. Levan, N.R. Tanvir, A.S. Fruchter, E. Rol, J.P.U. Fynbo, J. Hjorth, G. Williams, E. Bergeron, D. Bersier, M. Bremer, T. Grav, P. Jakobsson, K. Nilsson, E. Olszewski, R.S. Priddey, D. Rafferty, J. Rhoads, The faint afterglow and host galaxy of the short-hard GRB 060121. Astrophys. J. Lett. 648, 9-12 (2006). doi:10.1086/507625

A.J. Levan, P. Jakobsson, C. Hurkett, N.R. Tanvir, J. Gorosabel, P. Vreeswijk, E. Rol, R. Chapman, N. Gehrels, P.T. O’Brien, J.P. Osborne, R.S. Priddey, C. Kouveliotou, R. Starling, D. vanden Berk, K. Wiersema, A case of mistaken identity? GRB060912A and the nature of the long-short GRB divide. Mon. Not. R. Astron. Soc. 378, 1439-1446 (2007). doi:10.1111/j.1365-2966.2007.11879.x

A.J. Levan, N.R. Tanvir, P. Jakobsson, R. Chapman, J. Hjorth, R.S. Priddey, J.P.U. Fynbo, K. Hurley, B.L. Jensen, R. Johnson, J. Gorosabel, A.J. Castro-Tirado, M. Jarvis, D. Watson, K. Wiersema, On the nature of the short-duration GRB 050906. Mon. Not. R. Astron. Soc. 384, 541-547 (2008). doi:10.1111/j.1365-2966.2007.11953.x

A.J. Levan, N.R. Tanvir, S.B. Cenko, D.A. Perley, K. Wiersema, J.S. Bloom, A.S. Fruchter, A.d.U. Postigo, P.T. O’Brien, N. Butler, A.J. van der Horst, G. Leloudas, A.N. Morgan, K. Misra, G.C. Bower, J. Farihi, R.L. Tunnicliffe, M. Modjaz, J.M. Silverman, J. Hjorth, C. Thöne, A. Cucchiara, J.M.C. Cerón, A.J. Castro-Tirado, J.A. Arnold, M. Bremer, J.P. Brodie, T. Carroll, M.C. Cooper, P.A. Curran, R.M. Cutri, J. Ehle, D. Forbes, J. Fynbo, J. Gorosabel, J. Graham, D.I. Hoffman, S. Guziy, P. Jakobsson, A. Kamble, T. Kerr, M.M. Kasliwal, C. Kouveliotou, D. Kocevski, N.M. Law, P.E. Nugent, E.O. Ofek, D. Poznanski, R.M. Quimby, E. Rol, A.J. Romanowsky, R. Sánchez-Ramírez, S. Schulze, N. Singh, L. van Spaandonk, R.L.C. Starling, R.G. Strom, J.C. Tello, O. Vaduvescu, P.J. Wheatley, R.A.M.J. Wijers, J.M. Winters, D. Xu, An extremely luminous panchromatic outburst from the nucleus of a distant galaxy. Science 333, 199 (2011). doi:10.1126/science.1207143

A.J. Levan, N.R. Tanvir, R.L.C. Starling, K. Wiersema, K.L. Page, D.A. Perley, S. Schulze, G.A. Wynn, R. Chornock, J. Hjorth, S.B. Cenko, A.S. Fruchter, P.T. O’Brien, G.C. Brown, R.L. Tunnicliffe, D. Malesani, P. Jakobsson, D. Watson, E. Berger, D. Bersier, B.E. Cobb, S. Covino, A. Cucchiara, A. de Ugarte Postigo, D.B. Fox, A. Gal-Yam, P. Goldoni, J. Gorosabel, L. Kaper, T. Krühler, R. Karjalainen, J.P. Osborne, E. Pian, R. Sánchez-Ramírez, B. Schmidt, I. Skillen, G. Tagliaferri, C. Thöne, O. Vaduvescu, R.A.M.J. Wijers, B.A. Zauderer, A new population of ultra-long duration gamma-ray bursts. Astrophys. J. 781, 13 (2014a). doi:10.1088/0004-637X/781/1/13

A.J. Levan, N.R. Tanvir, A.S. Fruchter, J. Hjorth, E. Pian, P. Mazzali, R.A. Hounsell, D.A. Perley, Z. Cano, J. Graham, S.B. Cenko, J.P.U. Fynbo, C. Kouveliotou, A. Pe'er, K. Misra, K. Wiersema, Hubble space telescope observations of the afterglow, supernova, and host galaxy associated with the extremely bright GRB 130427A. Astrophys. J. 792, 115 (2014b). doi:10.1088/0004-637X/792/2/115

A. Levan, P. Nugent, A. Fruchter, I. Burud, D. Branch, J. Rhoads, A. Castro-Tirado, J. Gorosabel, J.M. Castro Cerón, S.E. Thorsett, C. Kouveliotou, S. Golenetskii, J. Fynbo, P. Garnavich, S. Holland, J. Hjorth, P. Møller, E. Pian, N. Tanvir, M. Ulanov, R. Wijers, S. Woosley, GRB 020410: a gamma-ray burst afterglow discovered by its supernova light. Astrophys. J. 624, 880-888 (2005). doi:10.1086/428657

E.M. Levesque, P. Massey, A.N. Żytkow, N. Morrell, Discovery of a Thorne-Żytkow object candidate in the Small Magellanic Cloud. Mon. Not. R. Astron. Soc. 443, 94-98 (2014). doi:10.1093/mnrasl/slu080

C. Li, R. de Grijs, L. Deng, The binary fractions in the massive young Large Magellanic Cloud star clusters NGC 1805 and NGC 1818. Mon. Not. R. Astron. Soc. 436, 1497-1512 (2013). doi:10.1093/mnras/ stt1669

C. Li, R. de Grijs, L. Deng, Not-so-simple stellar populations in the intermediate-age Large Magellanic Cloud star clusters NGC 1831 and NGC 1868. Astrophys. J. 784, 157 (2014). doi:10.1088/0004-637X/ $784 / 2 / 157$

C. Li, R. de Grijs, L. Deng, X. Liu, Blue straggler evolution caught in the act in the Large Magellanic Cloud globular cluster Hodge 11. Astrophys. J. Lett. 770, 7 (2013). doi:10.1088/2041-8205/770/1/L7

L.-X. Li, B. Paczyński, Transient events from neutron star mergers. Astrophys. J. Lett. 507, 59-62 (1998). doi: $10.1086 / 311680$

E. Liang, B. Zhang, F. Virgili, Z.G. Dai, Low-luminosity gamma-ray bursts as a unique population: luminosity function, local rate, and beaming factor. Astrophys. J. 662, 1111-1118 (2007a). doi:10.1086/517959

E. Liang, B. Zhang, F. Virgili, Z.G. Dai, Low-luminosity gamma-ray bursts as a unique population: luminosity function, local rate, and beaming factor. Astrophys. J. 662, 1111-1118 (2007b). doi:10.1086/517959

Y.M. Lipkin, E.O. Ofek, A. Gal-Yam, E.M. Leibowitz, D. Poznanski, S. Kaspi, D. Polishook, S.R. Kulkarni, D.W. Fox, E. Berger, N. Mirabal, J. Halpern, M. Bureau, K. Fathi, P.A. Price, B.A. Peterson, A. Frebel, B. Schmidt, J.A. Orosz, J.B. Fitzgerald, J.S. Bloom, P.G. van Dokkum, C.D. Bailyn, M.M. Buxton, M. Barsony, The detailed optical light curve of GRB 030329. Astrophys. J. 606, 381-394 (2004). doi: $10.1086 / 383000$

D.R. Lorimer, M. Kramer, Handbook of Pulsar Astronomy (2004) 
R. Lunnan, R. Chornock, E. Berger, T. Laskar, W. Fong, A. Rest, N.E. Sanders, P.M. Challis, M.R. Drout, R.J. Foley, M.E. Huber, R.P. Kirshner, C. Leibler, G.H. Marion, M. McCrum, D. Milisavljevic, G. Narayan, D. Scolnic, S.J. Smartt, K.W. Smith, A.M. Soderberg, J.L. Tonry, W.S. Burgett, K.C. Chambers, H. Flewelling, K.W. Hodapp, N. Kaiser, E.A. Magnier, P.A. Price, R.J. Wainscoat, Hydrogen-poor superluminous supernovae and long-duration gamma-ray bursts have similar host galaxies. Astrophys. J. 787, 138 (2014). doi:10.1088/0004-637X/787/2/138

R. Lunnan, R. Chornock, E. Berger, A. Rest, W. Fong, D. Scolnic, D.O. Jones, A.M. Soderberg, P.M. Challis, M.R. Drout, R.J. Foley, M.E. Huber, R.P. Kirshner, C. Leibler, G.H. Marion, M. McCrum, D. Milisavljevic, G. Narayan, N.E. Sanders, S.J. Smartt, K.W. Smith, J.L. Tonry, W.S. Burgett, K.C. Chambers, H. Flewelling, R.-P. Kudritzki, R.J. Wainscoat, C. Waters, Zooming in on the progenitors of superluminous supernovae with the HST. Astrophys. J. 804, 90 (2015). doi:10.1088/0004-637X/804/2/90

J.D. Lyman, D. Bersier, P.A. James, Bolometric corrections for optical light curves of core-collapse supernovae. Mon. Not. R. Astron. Soc. 437, 3848-3862 (2014a). doi:10.1093/mnras/stt2187

J.D. Lyman, A.J. Levan, R.P. Church, M.B. Davies, N.R. Tanvir, The progenitors of calcium-rich transients are not formed in situ. Mon. Not. R. Astron. Soc. 444, 2157-2166 (2014b). doi:10.1093/mnras/stu 1574

J.D. Lyman, D. Bersier, P.A. James, P.A. Mazzali, J.J. Eldridge, M. Fraser, E. Pian, Bolometric light curves and explosion parameters of 38 stripped-envelope core-collapse supernovae. Mon. Not. R. Astron. Soc. 457, 328-350 (2016a). doi:10.1093/mnras/stv2983

J.D. Lyman, A.J. Levan, P.A. James, C.R. Angus, R.P. Church, M.B. Davies, N.R. Tanvir, Hubble Space Telescope observations of the host galaxies and environments of calcium-rich supernovae. Mon. Not. R. Astron. Soc. 458, 1768-1777 (2016b). doi:10.1093/mnras/stw477

T.J. Maccarone, S.E. de Mink, Large proper motion of the Thorne-Żytkow object candidate HV 2112 reveals its likely nature as foreground Galactic S-star. Mon. Not. R. Astron. Soc. 458, 1-5 (2016). doi:10.1093/mnrasl/slw004

b.P. Macias, E. Ramirez-Ruiz, A Stringent Limit on the Mass Production Rate of $r$-Process Elements in the Milky Way. ArXiv e-prints (2016)

K. Maeda, T. Nakamura, K. Nomoto, P.A. Mazzali, F. Patat, I. Hachisu, Explosive nucleosynthesis in aspherical hypernova explosions and late-time spectra of SN 1998bw. Astrophys. J. 565, 405-412 (2002). doi:10.1086/324487

F. Martins, D.J. Hillier, J.C. Bouret, E. Depagne, C. Foellmi, S. Marchenko, A.F. Moffat, Properties of WNh stars in the Small Magellanic Cloud: evidence for homogeneous evolution. Astron. Astrophys. 495, 257-270 (2009). doi:10.1051/0004-6361:200811014

A. Maselli, A. Melandri, L. Nava, C.G. Mundell, N. Kawai, S. Campana, S. Covino, J.R. Cummings, G. Cusumano, P.A. Evans, G. Ghirlanda, G. Ghisellini, C. Guidorzi, S. Kobayashi, P. Kuin, V. La Parola, V. Mangano, S. Oates, T. Sakamoto, M. Serino, F. Virgili, B.-B. Zhang, S. Barthelmy, A. Beardmore, M.G. Bernardini, D. Bersier, D. Burrows, G. Calderone, M. Capalbi, J. Chiang, P. D’Avanzo, V. D'Elia, M. De Pasquale, D. Fugazza, N. Gehrels, A. Gomboc, R. Harrison, H. Hanayama, J. Japelj, J. Kennea, D. Kopac, C. Kouveliotou, D. Kuroda, A. Levan, D. Malesani, F. Marshall, J. Nousek, P. O’Brien, J.P. Osborne, C. Pagani, K.L. Page, M. Page, M. Perri, T. Pritchard, P. Romano, Y. Saito, B. Sbarufatti, R. Salvaterra, I. Steele, N. Tanvir, G. Vianello, B. Weigand, K. Wiersema, Y. Yatsu, T. Yoshii, G. Tagliaferri, GRB 130427A: a nearby ordinary monster. Science 343, 48-51 (2014). doi:10.1126/ science. 1242279

N. Masetti, E. Palazzi, E. Pian, A. Simoncelli, L.K. Hunt, E. Maiorano, A. Levan, L. Christensen, E. Rol, S. Savaglio, R. Falomo, A.J. Castro-Tirado, J. Hjorth, A. Delsanti, M. Pannella, V. Mohan, S.B. Pandey, R. Sagar, L. Amati, I. Burud, J.M. Castro Cerón, F. Frontera, A.S. Fruchter, J.P.U. Fynbo, J. Gorosabel, L. Kaper, S. Klose, C. Kouveliotou, L. Nicastro, H. Pedersen, J. Rhoads, I. Salamanca, N. Tanvir, P.M. Vreeswijk, R.A.M.J. Wijers, E.P.J. van den Heuvel, Optical and near-infrared observations of the GRB020405 afterglow. Astron. Astrophys. 404, 465-481 (2003). doi:10.1051/0004-6361:20030491

B.D. Mason, T.J. Henry, W.I. Hartkopf, T. ten Brummelaar, D.R. Soderblom, A multiplicity survey of chromospherically active and inactive stars. Astron. J. 116, 2975-2983 (1998). doi:10.1086/300654

E.P. Mazets, S.V. Golenetskii, V.N. Ilinskii, V.N. Panov, R.L. Aptekar, I.A. Gurian, M.P. Proskura, I.A. Sokolov, Z.I. Sokolova, T.V. Kharitonova, Catalog of cosmic gamma-ray bursts from the KONUS experiment data. I. Astrophys. Space Sci. 80, 3-83 (1981). doi:10.1007/BF00649140

E.P. Mazets, S.V. Golenetskii, I.A. Gurian, V.N. Ilinskii, The 5 March 1979 event and the distinct class of short gamma bursts are they of the same origin. Astrophys. Space Sci. 84, 173-189 (1982). doi:10.1007/ BF00713635

P.A. Mazzali, J. Deng, K. Nomoto, D.N. Sauer, E. Pian, N. Tominaga, M. Tanaka, K. Maeda, A.V. Filippenko, A neutron-star-driven X-ray flash associated with supernova SN 2006aj. Nature 442, 1018-1020 (2006). doi:10.1038/nature05081

P.A. Mazzali, R.J. Foley, J. Deng, F. Patat, E. Pian, D. Baade, J.S. Bloom, A.V. Filippenko, D.A. Perley, S. Valenti, L. Wang, K. Kawabata, K. Maeda, K-i. Nomoto, Keck and European Southern Observatory 
very large telescope view of the symmetry of the ejecta of the XRF/SN 2006aj. Astrophys. J. 661, 892-898 (2007a). doi:10.1086/517912

P.A. Mazzali, K.S. Kawabata, K. Maeda, R.J. Foley, K. Nomoto, J. Deng, T. Suzuki, M. Iye, N. Kashikawa, Y. Ohyama, A.V. Filippenko, Y. Qiu, J. Wei, The aspherical properties of the energetic type Ic SN 2002ap as inferred from its nebular spectra. Astrophys. J. 670, 592-599 (2007b). doi:10.1086/521873

P.A. Mazzali, K.S. Kawabata, K. Maeda, R.J. Foley, K. Nomoto, J. Deng, T. Suzuki, M. Iye, N. Kashikawa, Y. Ohyama, A.V. Filippenko, Y. Qiu, J. Wei, The aspherical properties of the energetic type Ic SN 2002ap as inferred from its nebular spectra. Astrophys. J. 670, 592-599 (2007c). doi:10.1086/521873

P.A. Mazzali, A.I. McFadyen, S.E. Woosley, E. Pian, M. Tanaka, An upper limit to the energy of gammaray bursts indicates that GRBs/SNe are powered by magnetars. Mon. Not. R. Astron. Soc. 443, 67-71 (2014). doi:10.1093/mnras/stu1124

P.A. Mazzali, M. Sullivan, E. Pian, J. Greiner, D.A. Kann, Spectrum formation in superluminous supernovae (Type I). Mon. Not. R. Astron. Soc. 458, 3455-3465 (2016). doi:10.1093/mnras/stw512

A. Melandri, E. Pian, V. D’Elia, P. D’Avanzo, M. Della Valle, P.A. Mazzali, G. Tagliaferri, Z. Cano, A.J. Levan, Diversity of gamma-ray burst energetics vs. supernova homogeneity: SN $2013 \mathrm{cq}$ associated with GRB 130427A. Astron. Astrophys. 567, 29 (2014). doi:10.1051/0004-6361/201423572

B.D. Metzger, Nuclear-dominated accretion and subluminous supernovae from the merger of a white dwarf with a neutron star or black hole. Mon. Not. R. Astron. Soc. 419, 827-840 (2012). doi:10.1111/ j.1365-2966.2011.19747.x

B.D. Metzger, E. Berger, What is the most promising electromagnetic counterpart of a neutron star binary merger? Astrophys. J. 746, 48 (2012). doi:10.1088/0004-637X/746/1/48

B.D. Metzger, D. Giannios, T.A. Thompson, N. Bucciantini, E. Quataert, The protomagnetar model for gamma-ray bursts. Mon. Not. R. Astron. Soc. 413, 2031-2056 (2011). doi:10.1111/j.1365-2966.2011. 18280.x

B.D. Metzger, B. Margalit, D. Kasen, E. Quataert, The diversity of transients from magnetar birth in core collapse supernovae. Mon. Not. R. Astron. Soc. 454, 3311-3316 (2015). doi:10.1093/mnras/stv2224

M.R. Mokiem, A. de Koter, C.J. Evans, J. Puls, S.J. Smartt, P.A. Crowther, A. Herrero, N. Langer, D.J. Lennon, F. Najarro, M.R. Villamariz, S.-C. Yoon, The VLT-FLAMES survey of massive stars: mass loss and rotation of early-type stars in the SMC. Astron. Astrophys. 456, 1131-1151 (2006). doi:10. 1051/0004-6361:20064995

S. Mukherjee, E.D. Feigelson, G. Jogesh Babu, F. Murtagh, C. Fraley, A. Raftery, Three types of gamma-ray bursts. Astrophys. J. 508, 314-327 (1998). doi:10.1086/306386

R. Narayan, B. Paczynski, T. Piran, Gamma-ray bursts as the death throes of massive binary stars. Astrophys. J. Lett. 395, 83-86 (1992). doi:10.1086/186493

G. Nelemans, C.A. Tout, Reconstructing the evolution of white dwarf binaries: further evidence for an alternative algorithm for the outcome of the common-envelope phase in close binaries. Mon. Not. R. Astron. Soc. 356, 753-764 (2005). doi:10.1111/j.1365-2966.2004.08496.x

R.J. Nemiroff, A century of gamma ray burst models. Comments Astrophys. 17, 189 (1994)

S. Nissanke, J. Sievers, N. Dalal, D. Holz, Localizing compact binary inspirals on the sky using ground-based gravitational wave interferometers. Astrophys. J. 739, 99 (2011). doi:10.1088/0004-637X/739/2/99

J.A. Nousek, C. Kouveliotou, D. Grupe, K.L. Page, J. Granot, E. Ramirez-Ruiz, S.K. Patel, D.N. Burrows, V. Mangano, S. Barthelmy, A.P. Beardmore, S. Campana, M. Capalbi, G. Chincarini, G. Cusumano, A.D. Falcone, N. Gehrels, P. Giommi, M.R. Goad, O. Godet, C.P. Hurkett, J.A. Kennea, A. Moretti, P.T. O’Brien, J.P. Osborne, P. Romano, G. Tagliaferri, A.A. Wells, Evidence for a canonical gammaray burst afterglow light curve in the Swift XRT data. Astrophys. J. 642, 389-400 (2006). doi:10.1086/ 500724

M. Nysewander, A.S. Fruchter, A. Pe'er, A comparison of the afterglows of short- and long-duration gammaray bursts. Astrophys. J. 701, 824-836 (2009a). doi:10.1088/0004-637X/701/1/824

M. Nysewander, A.S. Fruchter, A. Pe'er, A comparison of the afterglows of short- and long-duration gammaray bursts. Astrophys. J. 701, 824-836 (2009b). doi:10.1088/0004-637X/701/1/824

R. Oechslin, H.-T. Janka, Torus formation in neutron star mergers and well-localized short gamma-ray bursts. Mon. Not. R. Astron. Soc. 368, 1489-1499 (2006). doi:10.1111/j.1365-2966.2006.10238.x

S.A. Olausen, V.M. Kaspi, The McGill magnetar catalog. Astrophys. J. Suppl. Ser. 212, 6 (2014). doi:10. 1088/0067-0049/212/1/6

D.M. Palmer, S. Barthelmy, N. Gehrels, R.M. Kippen, T. Cayton, C. Kouveliotou, D. Eichler, R.A.M.J. Wijers, P.M. Woods, J. Granot, Y.E. Lyubarsky, E. Ramirez-Ruiz, L. Barbier, M. Chester, J. Cummings, E.E. Fenimore, M.H. Finger, B.M. Gaensler, D. Hullinger, H. Krimm, C.B. Markwardt, J.A. Nousek, A. Parsons, S. Patel, T. Sakamoto, G. Sato, M. Suzuki, J. Tueller, A giant $\gamma$-ray flare from the magnetar SGR 1806-20. Nature 434, 1107-1109 (2005). doi:10.1038/nature03525

D.A. Perley, B.D. Metzger, J. Granot, N.R. Butler, T. Sakamoto, E. Ramirez-Ruiz, A.J. Levan, J.S. Bloom, A.A. Miller, A. Bunker, H.-W. Chen, A.V. Filippenko, N. Gehrels, K. Glazebrook, P.B. Hall, K.C. Hur- 
ley, D. Kocevski, W. Li, S. Lopez, J. Norris, A.L. Piro, D. Poznanski, J.X. Prochaska, E. Quataert, N. Tanvir, GRB 080503: implications of a naked short gamma-ray burst dominated by extended emission. Astrophys. J. 696, 1871-1885 (2009). doi:10.1088/0004-637X/696/2/1871

D.A. Perley, S.B. Cenko, A. Corsi, N.R. Tanvir, A.J. Levan, D.A. Kann, E. Sonbas, K. Wiersema, W. Zheng, X.-H. Zhao, J.-M. Bai, M. Bremer, A.J. Castro-Tirado, L. Chang, K.I. Clubb, D. Frail, A. Fruchter, E. Göğüş, J. Greiner, T. Güver, A. Horesh, A.V. Filippenko, S. Klose, J. Mao, A.N. Morgan, A.S. Pozanenko, S. Schmidl, B. Stecklum, M. Tanga, A.A. Volnova, A.E. Volvach, J.-G. Wang, J.-M. Winters, Y.-X. Xin, The afterglow of GRB 130427A from 1 to $10^{16} \mathrm{GHz}$. Astrophys. J. 781, 37 (2014). doi:10. 1088/0004-637X/781/1/37

D.A. Perley, N.R. Tanvir, J. Hjorth, T. Laskar, E. Berger, R. Chary, A. de Ugarte Postigo, J.P.U. Fynbo, T. Krühler, A.J. Levan, M.J. Michałowski, S. Schulze, The Swift GRB Host Galaxy Legacy Survey-II. Rest-Frame NIR Luminosity Distribution and Evidence for a Near-Solar Metallicity Threshold. ArXiv e-prints (2015)

D.A. Perley, T. Krühler, P. Schady, M.J. Michałowski, C.C. Thöne, D. Petry, J.F. Graham, J. Greiner, S. Schulze, S. Kim, A Revised Host Galaxy Association for GRB 020819B: A High-Redshift Dusty Starburst, Not a Low-Redshift Gas-Poor Spiral. ArXiv e-prints (2016)

R. Perna, P.J. Armitage, B. Zhang, Flares in long and short gamma-ray bursts: a common origin in a hyperaccreting accretion disk. Astrophys. J. Lett. 636, 29-32 (2006a). doi:10.1086/499775

E. Pian, P.A. Mazzali, N. Masetti, P. Ferrero, S. Klose, E. Palazzi, E. Ramirez-Ruiz, S.E. Woosley, C. Kouveliotou, J. Deng, A.V. Filippenko, R.J. Foley, J.P.U. Fynbo, D.A. Kann, W. Li, J. Hjorth, K. Nomoto, F. Patat, D.N. Sauer, J. Sollerman, P.M. Vreeswijk, E.W. Guenther, A. Levan, P. O’Brien, N.R. Tanvir, R.A.M.J. Wijers, C. Dumas, O. Hainaut, D.S. Wong, D. Baade, L. Wang, L. Amati, E. Cappellaro, A.J. Castro-Tirado, S. Ellison, F. Frontera, A.S. Fruchter, J. Greiner, K. Kawabata, C. Ledoux, K. Maeda, P. Møller, L. Nicastro, E. Rol, R. Starling, An optical supernova associated with the X-ray flash XRF 060218. Nature 442, 1011-1013 (2006b). doi:10.1038/nature05082

P. Podsiadlowski, P.A. Mazzali, K. Nomoto, D. Lazzati, E. Cappellaro, The rates of hypernovae and gammaray bursts: implications for their progenitors. Astrophys. J. Lett. 607, 17-20 (2004). doi:10.1086/421347

P. Podsiadlowski, N. Ivanova, S. Justham, S. Rappaport, Explosive common-envelope ejection: implications for gamma-ray bursts and low-mass black-hole binaries. Mon. Not. R. Astron. Soc. 406, 840-847 (2010). doi:10.1111/j.1365-2966.2010.16751.x

S.F. Portegies Zwart, S.L.W. McMillan, M. Gieles, Young massive star clusters. Annu. Rev. Astron. Astrophys. 48, 431-493 (2010). doi:10.1146/annurev-astro-081309-130834

S. Prajs, M. Sullivan, M. Smith, A. Levan, N.V. Karpenka, T.D.P. Edwards, C.R. Walker, W.M. Wolf, C. Balland, R. Carlberg, A. Howell, C. Lidman, R. Pain, C. Pritchet, V. Ruhlmann-Kleider, The Volumetric Rate of Superluminous Supernovae at $\mathrm{z} \sim 1$. ArXiv e-prints (2016)

P.A. Price, E. Berger, D.E. Reichart, S.R. Kulkarni, S.A. Yost, R. Subrahmanyan, R.M. Wark, M.H. Wieringa, D.A. Frail, J. Bailey, B. Boyle, E. Corbett, K. Gunn, S.D. Ryder, N. Seymour, K. Koviak, P. McCarthy, M. Phillips, T.S. Axelrod, J.S. Bloom, S.G. Djorgovski, D.W. Fox, T.J. Galama, F.A. Harrison, K. Hurley, R. Sari, B.P. Schmidt, M.J.I. Brown, T. Cline, F. Frontera, C. Guidorzi, E. Montanari, GRB 011121: a massive star progenitor. Astrophys. J. Lett. 572, 51-55 (2002). doi:10.1086/341552

P.A. Price, S.R. Kulkarni, E. Berger, D.W. Fox, J.S. Bloom, S.G. Djorgovski, D.A. Frail, T.J. Galama, F.A. Harrison, P. McCarthy, D.E. Reichart, R. Sari, S.A. Yost, H. Jerjen, K. Flint, A. Phillips, B.E. Warren, T.S. Axelrod, R.A. Chevalier, J. Holtzman, R.A. Kimble, B.P. Schmidt, J.C. Wheeler, F. Frontera, E. Costa, L. Piro, K. Hurley, T. Cline, C. Guidorzi, E. Montanari, E. Mazets, S. Golenetskii, I. Mitrofanov, D. Anfimov, A. Kozyrev, M. Litvak, A. Sanin, W. Boynton, C. Fellows, K. Harshman, C. Shinohara, A. Gal-Yam, E. Ofek, Y. Lipkin, Discovery of GRB 020405 and its late red bump. Astrophys. J. 589, 838-843 (2003a). doi:10.1086/374730

P.A. Price, S.R. Kulkarni, B.P. Schmidt, T.J. Galama, J.S. Bloom, E. Berger, D.A. Frail, S.G. Djorgovski, D.W. Fox, A.A. Henden, S. Klose, F.A. Harrison, D.E. Reichart, R. Sari, S.A. Yost, T.S. Axelrod, P. McCarthy, J. Holtzman, J.P. Halpern, R.A. Kimble, J.C. Wheeler, R.A. Chevalier, K. Hurley, G.R. Ricker, E. Costa, F. Frontera, L. Piro, GRB 010921: strong limits on an underlying supernova from the Hubble Space Telescope. Astrophys. J. 584, 931-936 (2003b). doi:10.1086/345734

P.A. Price, D.W. Fox, S.R. Kulkarni, B.A. Peterson, B.P. Schmidt, A.M. Soderberg, S.A. Yost, E. Berger, S.G. Djorgovski, D.A. Frail, F.A. Harrison, R. Sari, A.W. Blain, S.C. Chapman, The bright optical afterglow of the nearby $\gamma$-ray burst of 29 March 2003. Nature 423, 844-847 (2003c). doi:10.1038/ nature 01734

R.M. Quimby, F. Yuan, C. Akerlof, J.C. Wheeler, Rates of superluminous supernovae at z=0.2. Mon. Not. R. Astron. Soc. 431, 912-922 (2013). doi:10.1093/mnras/stt213

D. Raghavan, H.A. McAlister, T.J. Henry, D.W. Latham, G.W. Marcy, B.D. Mason, D.R. Gies, R.J. White, T.A. ten Brummelaar, A survey of stellar families: multiplicity of solar-type stars. Astrophys. J. Suppl. Ser. 190, 1-42 (2010). doi:10.1088/0067-0049/190/1/1 
O.H. Ramírez-Agudelo, H. Sana, S.E. de Mink, V. Hénault-Brunet, A. de Koter, N. Langer, F. Tramper, G. Gräfener, C.J. Evans, J.S. Vink, P.L. Dufton, W.D. Taylor, The VLT-FLAMES Tarantula Survey. XXI. Stellar spin rates of O-type spectroscopic binaries. Astron. Astrophys. 580, 92 (2015). doi:10. 1051/0004-6361/201425424

C. Raskin, E. Scannapieco, J. Rhoads, M. Della Valle, Using spatial distributions to constrain progenitors of supernovae and gamma-ray bursts. Astrophys. J. 689, 358-370 (2008). doi:10.1086/592495

N. Rea, M. Gullón, J.A. Pons, R. Perna, M.G. Dainotti, J.A. Miralles, D.F. Torres, Constraining the GRBmagnetar model by means of the galactic pulsar population. Astrophys. J. 813, 92 (2015). doi:10.1088/ 0004-637X/813/2/92

T.M. Reynolds, M. Fraser, G. Gilmore, Gone without a bang: an archival HST survey for disappearing massive stars. Mon. Not. R. Astron. Soc. 453(3), 2889-2900 (2015)

J.E. Rhoads, The dynamics and light curves of beamed gamma-ray burst afterglows. Astrophys. J. 525, 737749 (1999). doi:10.1086/307907

S. Rosswog, E. Ramirez-Ruiz, M.B. Davies, High-resolution calculations of merging neutron stars-III. Gamma-ray bursts. Mon. Not. R. Astron. Soc. 345, 1077-1090 (2003). doi:10.1046/j.1365-2966.2003. 07032.x

S. Rosswog, M. Liebendörfer, F.-K. Thielemann, M.B. Davies, W. Benz, T. Piran, Mass ejection in neutron star mergers. Astron. Astrophys. 341, 499-526 (1999).

A. Rowlinson, P.T. O’Brien, B.D. Metzger, N.R. Tanvir, A.J. Levan, Signatures of magnetar central engines in short GRB light curves. Mon. Not. R. Astron. Soc. 430, 1061-1087 (2013). doi:10.1093/mnras/sts683

H. Sana, G. James, E. Gosset, The massive star binary fraction in young open clusters-III. IC 2944 and the Cen OB2 association. Mon. Not. R. Astron. Soc. 416, 817 (2011)

H. Sana, Y. Momany, M. Gieles, G. Carraro, Y. Beletsky, V.D. Ivanov, G. de Silva, G. James, A MAD view of Trumpler 14. Astron. Astrophys. 515, 26 (2010). doi:10.1051/0004-6361/200913688

H. Sana, S.E. de Mink, A. de Koter, N. Langer, C.J. Evans, M. Gieles, E. Gosset, R.G. Izzard, J.-B. Le Bouquin, F.R.N. Schneider, Binary interaction dominates the evolution of massive stars. Science 337, 444 (2012). doi:10.1126/science.1223344

H. Sana, A. de Koter, S.E. de Mink, P.R. Dunstall, C.J. Evans, V. Hénault-Brunet, J. Maíz Apellániz, O.H. Ramírez-Agudelo, W.D. Taylor, N.R. Walborn, J.S. Clark, P.A. Crowther, A. Herrero, M. Gieles, N. Langer, D.J. Lennon, J.S. Vink, The VLT-FLAMES Tarantula Survey. VIII. Multiplicity properties of the O-type star population. Astron. Astrophys. 550, 107 (2013). doi:10.1051/0004-6361/201219621

R. Sari, T. Piran, R. Narayan, Spectra and light curves of gamma-ray burst afterglows. Astrophys. J. Lett. 497, 17-20 (1998). doi:10.1086/311269

S. Savaglio, K. Glazebrook, D. Le Borgne, The galaxy population hosting gamma-ray bursts. Astrophys. J. 691, 182-211 (2009). doi:10.1088/0004-637X/691/1/182

b.T. Shenar, R. Hainich, H. Todt, A. Sander, W.-R. Hamann, A.F.J. Moffat, J.J. Eldridge, H. Pablo, L.M. Oskinova, N.D. Richardson, Wolf-Rayet stars in the Small Magellanic Cloud: II. Analysis of the binaries. ArXiv e-prints (2016)

S.J. Smartt, J.J. Eldridge, R.M. Crockett, J.R. Maund, The death of massive stars-I. Observational constraints on the progenitors of Type II-P supernovae. Mon. Not. R. Astron. Soc. 395, 1409-1437 (2009). doi:10.1111/j.1365-2966.2009.14506.x

A.M. Soderberg, S.R. Kulkarni, E. Berger, D.W. Fox, M. Sako, D.A. Frail, A. Gal-Yam, D.S. Moon, S.B. Cenko, S.A. Yost, M.M. Phillips, S.E. Persson, W.L. Freedman, P. Wyatt, R. Jayawardhana, D. Paulson, The sub-energetic $\gamma$-ray burst GRB 031203 as a cosmic analogue to the nearby GRB 980425 . Nature 430, 648-650 (2004). doi:10.1038/nature02757

A.M. Soderberg, S.R. Kulkarni, E. Nakar, E. Berger, P.B. Cameron, D.B. Fox, D. Frail, A. Gal-Yam, R. Sari, S.B. Cenko, M. Kasliwal, R.A. Chevalier, T. Piran, P.A. Price, B.P. Schmidt, G. Pooley, D.-S. Moon, B.E. Penprase, E. Ofek, A. Rau, N. Gehrels, J.A. Nousek, D.N. Burrows, S.E. Persson, P.J. McCarthy, Relativistic ejecta from X-ray flash XRF 060218 and the rate of cosmic explosions. Nature 442, 10141017 (2006). doi:10.1038/nature05087

M. Sparre, R.L.C. Starling, A search for thermal X-ray signatures in gamma-ray bursts-II. The Swift sample. Mon. Not. R. Astron. Soc. 427, 2965-2974 (2012). doi:10.1111/j.1365-2966.2012.21858.x

H.C. Spruit, Dynamo action by differential rotation in a stably stratified stellar interior. Astron. Astrophys. 381, 923-932 (2002). doi:10.1051/0004-6361:20011465

K.Z. Stanek, T. Matheson, P.M. Garnavich, P. Martini, P. Berlind, N. Caldwell, P. Challis, W.R. Brown, R. Schild, K. Krisciunas, M.L. Calkins, J.C. Lee, N. Hathi, R.A. Jansen, R. Windhorst, L. Echevarria, D.J. Eisenstein, B. Pindor, E.W. Olszewski, P. Harding, S.T. Holland, D. Bersier, Spectroscopic discovery of the supernova 2003dh associated with GRB 030329. Astrophys. J. Lett. 591, 17-20 (2003). doi: $10.1086 / 376976$

E.R. Stanway, J.J. Eldridge, G.D. Becker, Stellar population effects on the inferred photon density at reionization. Mon. Not. R. Astron. Soc. 456, 485-499 (2016). doi:10.1093/mnras/stv2661 
R.L.C. Starling, K. Wiersema, A.J. Levan, T. Sakamoto, D. Bersier, P. Goldoni, S.R. Oates, A. Rowlinson, S. Campana, J. Sollerman, N.R. Tanvir, D. Malesani, J.P.U. Fynbo, S. Covino, P. D’Avanzo, P.T. O’Brien, K.L. Page, J.P. Osborne, S.D. Vergani, S. Barthelmy, D.N. Burrows, Z. Cano, P.A. Curran, M. de Pasquale, V. D’Elia, P.A. Evans, H. Flores, A.S. Fruchter, P. Garnavich, N. Gehrels, J. Gorosabel, J. Hjorth, S.T. Holland, A.J. van der Horst, C.P. Hurkett, P. Jakobsson, A.P. Kamble, C. Kouveliotou, N.P.M. Kuin, L. Kaper, P.A. Mazzali, P.E. Nugent, E. Pian, M. Stamatikos, C.C. Thöne, S.E. Woosley, Discovery of the nearby long, soft GRB 100316D with an associated supernova. Mon. Not. R. Astron. Soc. 411, 2792-2803 (2011). doi:10.1111/j.1365-2966.2010.17879.x

R.L.C. Starling, K.L. Page, A. Pe'Er, A.P. Beardmore, J.P. Osborne, A search for thermal X-ray signatures in gamma-ray bursts-I. Swift bursts with optical supernovae. Mon. Not. R. Astron. Soc. 427, 2950-2964 (2012). doi:10.1111/j.1365-2966.2012.22116.x

M.P.L. Suijs, N. Langer, A.-J. Poelarends, S.-C. Yoon, A. Heger, F. Herwig, White dwarf spins from low-mass stellar evolution models. Astron. Astrophys. 481, 87-90 (2008). doi:10.1051/0004-6361:200809411

b.H. Sun, B. Zhang, H. Gao, X-ray counterpart of gravitational waves due to binary neutron star mergers: light curves, luminosity functions, and event rate densities. ArXiv e-prints (2016)

H. Sun, B. Zhang, Z. Li, Extragalactic high-energy transients: event rate densities and luminosity functions. Astrophys. J. 812, 33 (2015). doi:10.1088/0004-637X/812/1/33

K.M. Svensson, A.J. Levan, N.R. Tanvir, A.S. Fruchter, L.-G. Strolger, The host galaxies of core-collapse supernovae and gamma-ray bursts. Mon. Not. R. Astron. Soc. 405, 57-76 (2010). doi:10.1111/j.13652966.2010.16442.x

P.A. Sweet, The importance of rotation in stellar evolution. Mon. Not. R. Astron. Soc. 110, 548 (1950). doi:10.1093/mnras/110.6.548

D. Szécsi, N. Langer, S.-C. Yoon, D. Sanyal, S. de Mink, C.J. Evans, T. Dermine, Low-metallicity massive single stars with rotation. Evolutionary models applicable to I Zwicky 18. Astron. Astrophys. 581, 15 (2015). doi:10.1051/0004-6361/201526617

N.R. Tanvir, R. Chapman, A.J. Levan, R.S. Priddey, An origin in the local Universe for some short $\gamma$-ray bursts. Nature 438, 991-993 (2005). doi:10.1038/nature04310

N.R. Tanvir, D.B. Fox, A.J. Levan, E. Berger, K. Wiersema, J.P.U. Fynbo, A. Cucchiara, T. Krühler, N. Gehrels, J.S. Bloom, J. Greiner, P.A. Evans, E. Rol, F. Olivares, J. Hjorth, P. Jakobsson, J. Farihi, R. Willingale, R.L.C. Starling, S.B. Cenko, D. Perley, J.R. Maund, J. Duke, R.A.M.J. Wijers, A.J. Adamson, A. Allan, M.N. Bremer, D.N. Burrows, A.J. Castro-Tirado, B. Cavanagh, A. de Ugarte Postigo, M.A. Dopita, T.A. Fatkhullin, A.S. Fruchter, R.J. Foley, J. Gorosabel, J. Kennea, T. Kerr, S. Klose, H.A. Krimm, V.N. Komarova, S.R. Kulkarni, A.S. Moskvitin, C.G. Mundell, T. Naylor, K. Page, B.E. Penprase, M. Perri, P. Podsiadlowski, K. Roth, R.E. Rutledge, T. Sakamoto, P. Schady, B.P. Schmidt, A.M. Soderberg, J. Sollerman, A.W. Stephens, G. Stratta, T.N. Ukwatta, D. Watson, E. Westra, T. Wold, C. Wolf, A $\gamma$-ray burst at a redshift of $\mathrm{z} \sim 8.2$. Nature 461, 1254-1257 (2009). doi:10.1038/nature08459

N.R. Tanvir, A.J. Levan, A.S. Fruchter, J. Hjorth, R.A. Hounsell, K. Wiersema, R.L. Tunnicliffe, A 'kilonova' associated with the short-duration $\gamma$-ray burst GRB 130603B. Nature 500, 547-549 (2013). doi:10.1038/nature12505

C.C. Thöne, A. de Ugarte Postigo, C.L. Fryer, K.L. Page, J. Gorosabel, M.A. Aloy, D.A. Perley, C. Kouveliotou, H.T. Janka, P. Mimica, J.L. Racusin, H. Krimm, J. Cummings, S.R. Oates, S.T. Holland, M.H. Siegel, M. de Pasquale, E. Sonbas, M. Im, W.-K. Park, D.A. Kann, S. Guziy, L.H. García, A. Llorente, K. Bundy, C. Choi, H. Jeong, H. Korhonen, P. Kubànek, J. Lim, A. Moskvitin, T. MuñozDarias, S. Pak, I. Parrish, The unusual $\gamma$-ray burst GRB 101225A from a helium star/neutron star merger at redshift 0.33. Nature 480, 72-74 (2011a). doi:10.1038/nature10611

C.C. Thöne, S. Campana, D. Lazzati, A. de Ugarte Postigo, J.P.U. Fynbo, L. Christensen, A.J. Levan, M.A. Aloy, J. Hjorth, P. Jakobsson, E.M. Levesque, D. Malesani, B. Milvang-Jensen, P.W.A. Roming, N.R. Tanvir, K. Wiersema, M. Gladders, E. Wuyts, H. Dahle, Variable Ly $\alpha$ sheds light on the environment surrounding GRB 090426. Mon. Not. R. Astron. Soc. 414, 479-488 (2011b). doi:10.1111/ j.1365-2966.2011.18408.x

F. Tramper, S.M. Straal, D. Sanyal, H. Sana, A. de Koter, G. Gräfener, N. Langer, J.S. Vink, S.E. de Mink, L. Kaper, Massive stars on the verge of exploding: the properties of oxygen sequence Wolf-Rayet stars. Astron. Astrophys. 581, 110 (2015). doi:10.1051/0004-6361/201425390

C.A. Tremonti, T.M. Heckman, G. Kauffmann, J. Brinchmann, S. Charlot, S.D.M. White, M. Seibert, E.W. Peng, D.J. Schlegel, A. Uomoto, M. Fukugita, J. Brinkmann, The origin of the mass-metallicity relation: insights from 53,000 star-forming galaxies in the Sloan Digital Sky Survey. Astrophys. J. 613, 898-913 (2004). doi:10.1086/423264

R.L. Tunnicliffe, A.J. Levan, N.R. Tanvir, A. Rowlinson, D.A. Perley, J.S. Bloom, S.B. Cenko, P.T. O’Brien, B.E. Cobb, K. Wiersema, D. Malesani, A. de Ugarte Postigo, J. Hjorth, J.P.U. Fynbo, P. Jakobsson, On 
the nature of the 'hostless' short GRBs. Mon. Not. R. Astron. Soc. 437, 1495-1510 (2014). doi:10.1093/ mnras/stt1975

R. Tylenda, N. Soker, Eruptions of the V838 Mon type: stellar merger versus nuclear outburst models. Astron. Astrophys. 451, 223-236 (2006). doi:10.1051/0004-6361:20054201

R. Tylenda, M. Hajduk, T. Kamiński, A. Udalski, I. Soszyński, M.K. Szymański, M. Kubiak, G. Pietrzyński, R. Poleski, Ł. Wyrzykowski, K. Ulaczyk, V1309 Scorpii: merger of a contact binary. Astron. Astrophys. 528, 114 (2011). doi:10.1051/0004-6361/201016221

V.V. Usov, Millisecond pulsars with extremely strong magnetic fields as a cosmological source of gamma-ray bursts. Nature 357, 472-474 (1992). doi:10.1038/357472a0

E.P.J. van den Heuvel, S.-C. Yoon, Long gamma-ray burst progenitors: boundary conditions and binary models. Astrophys. Space Sci. 311, 177-183 (2007). doi:10.1007/s10509-007-9583-8

J. van Paradijs, P.J. Groot, T. Galama, C. Kouveliotou, R.G. Strom, J. Telting, R.G.M. Rutten, G.J. Fishman, C.A. Meegan, M. Pettini, N. Tanvir, J. Bloom, H. Pedersen, H.U. Nørdgaard-Nielsen, M. Linden-Vørnle, J. Melnick, G. van der Steene, M. Bremer, R. Naber, J. Heise, J. in't Zand, E. Costa, M. Feroci, L. Piro, F. Frontera, G. Zavattini, L. Nicastro, E. Palazzi, K. Bennett, L. Hanlon, A. Parmar, Transient optical emission from the error box of the $\gamma$-ray burst of 28 February 1997. Nature 386, 686-689 (1997). doi: $10.1038 / 386686 \mathrm{a} 0$

S. Vaughan, R. Willingale, P.T. O’Brien, J.P. Osborne, J.N. Reeves, A.J. Levan, M.G. Watson, J.A. Tedds, D. Watson, M. Santos-Lleó, P.M. Rodríguez-Pascual, N. Schartel, The discovery of an evolving dustscattered X-ray halo around GRB 031203. Astrophys. J. Lett. 603, 5-8 (2004). doi:10.1086/382785

J.S. Vink, Constraining GRB progenitor models by probing Wolf-Rayet wind geometries in the Large Magellanic Cloud. Astron. Astrophys. 469, 707-711 (2007). doi:10.1051/0004-6361:20077302

J.S. Vink, A. de Koter, On the metallicity dependence of Wolf-Rayet winds. Astron. Astrophys. 442, 587-596 (2005). doi:10.1051/0004-6361:20052862

J.S. Vink, A. de Koter, H.J.G.L.M. Lamers, Mass-loss predictions for O and B stars as a function of metallicity. Astron. Astrophys. 369, 574-588 (2001). doi:10.1051/0004-6361:20010127

A. Wallner, T. Faestermann, J. Feige, C. Feldstein, K. Knie, G. Korschinek, W. Kutschera, A. Ofan, M. Paul, F. Quinto, G. Rugel, P. Steier, Abundance of live ${ }^{244} \mathrm{Pu}$ in deep-sea reservoirs on Earth points to rarity of actinide nucleosynthesis. Nat. Commun. 6, 5956 (2015). doi:10.1038/ncomms6956

D. Watson, S.A. Vaughan, R. Willingale, J. Hjorth, S. Foley, J.P.U. Fynbo, P. Jakobsson, A. Levan, P.T. O’Brien, J.P. Osborne, K. Pedersen, J.N. Reeves, J.A. Tedds, M.G. Watson, The soft X-ray blast in the apparently subluminous GRB 031203. Astrophys. J. 636, 967-970 (2006). doi:10.1086/498089

C. Weidner, P. Kroupa, I.A.D. Bonnell, The relation between the most-massive star and its parental star cluster mass. Mon. Not. R. Astron. Soc. 401, 275-293 (2010). doi:10.1111/j.1365-2966.2009.15633.x

R. Willingale, J.P. Osborne, P.T. O’Brien, M.J. Ward, A. Levan, K.L. Page, The X-ray, optical and radio evolution of the GRB 030329 afterglow and the associated SN2003dh. Mon. Not. R. Astron. Soc. 349, 31-38 (2004). doi:10.1111/j.1365-2966.2004.07475.x

S.E. Woosley, Gamma-ray bursts from stellar mass accretion disks around black holes. Astrophys. J. 405, 273-277 (1993). doi:10.1086/172359

S.E. Woosley, A. Heger, The progenitor stars of gamma-ray bursts. Astrophys. J. 637, 914-921 (2006). doi: $10.1086 / 498500$

S.E. Woosley, R.G. Eastman, B.P. Schmidt, Gamma-ray bursts and Type IC supernova SN 1998BW. Astrophys. J. 516, 788-796 (1999). doi:10.1086/307131

C.C. Worley, M.J. Irwin, C.A. Tout, A.N. Żytkow, M. Fraser, R.G. Izzard, The proper motion of HV2112: a TŻO candidate in the SMC. Mon. Not. R. Astron. Soc. 459, 31-35 (2016). doi:10.1093/mnrasl/slw034

D. Xu, A. de Ugarte Postigo, G. Leloudas, T. Krühler, Z. Cano, J. Hjorth, D. Malesani, J.P.U. Fynbo, C.C. Thöne, R. Sánchez-Ramírez, S. Schulze, P. Jakobsson, L. Kaper, J. Sollerman, D.J. Watson, A. Cabrera-Lavers, C. Cao, S. Covino, H. Flores, S. Geier, J. Gorosabel, S.M. Hu, B. Milvang-Jensen, M. Sparre, L.P. Xin, T.M. Zhang, W.K. Zheng, Y.C. Zou, Discovery of the broad-lined Type Ic SN 2013cq associated with the very energetic GRB 130427A. Astrophys. J. 776, 98 (2013). doi:10.1088/ 0004-637X/776/2/98

S.-C. Yoon, N. Langer, Evolution of rapidly rotating metal-poor massive stars towards gamma-ray bursts. Astron. Astrophys. 443, 643-648 (2005). doi:10.1051/0004-6361:20054030

S.-C. Yoon, A. Dierks, N. Langer, Evolution of massive population III stars with rotation and magnetic fields. Astron. Astrophys. 542, 113 (2012). doi:10.1051/0004-6361/201117769

S.-C. Yoon, N. Langer, C. Norman, Single star progenitors of long gamma-ray bursts. I. Model grids and redshift dependent GRB rate. Astron. Astrophys. 460, 199-208 (2006). doi:10.1051/0004-6361:20065912

S.-C. Yoon, S.E. Woosley, N. Langer, Type Ib/c supernovae in binary systems. I. Evolution and properties of the progenitor stars. Astrophys. J. 725, 940-954 (2010). doi:10.1088/0004-637X/725/1/940 
N. Yusof, R. Hirschi, G. Meynet, P.A. Crowther, S. Ekström, U. Frischknecht, C. Georgy, H. Abu Kassim, O. Schnurr, Evolution and fate of very massive stars. Mon. Not. R. Astron. Soc. 433, 1114-1132 (2013). doi:10.1093/mnras/stt794

A. Zeh, S. Klose, D.H. Hartmann, A systematic analysis of supernova light in gamma-ray burst afterglows. Astrophys. J. 609, 952-961 (2004). doi:10.1086/421100

B. Zhang, B.-B. Zhang, F.J. Virgili, E.-W. Liang, D.A. Kann, X.-F. Wu, D. Proga, H.-J. Lv, K. Toma, P. Mészáros, D.N. Burrows, P.W.A. Roming, N. Gehrels, Discerning the physical origins of cosmological gamma-ray bursts based on multiple observational criteria: the cases of $\mathrm{z}=6.7$ GRB 080913, $\mathrm{z}=8.2$ GRB 090423, and some short/hard GRBs. Astrophys. J. 703, 1696-1724 (2009). doi:10.1088/ 0004-637X/703/2/1696 